\title{
A cortical hierarchy for differential GABAergic circuit motifs as targets for stress-induced alterations in endocannabinoid signaling
}

\author{
Dissertation \\ for the award of the degree \\ “Doctor rerum naturalium” (Dr. rer. nat.) \\ of the Georg-August Universität Göttingen
}

within the doctoral program Cellular and Molecular Physiology of the Brain of the Georg-August University School of Science (GAUSS)

submitted by

Karl-Alexander Engelhardt

from Hildesheim, Germany

Göttingen 2020 


\section{Thesis Committee}

\section{Dr. Dr. Oliver M. Schlüter}

Department of Psychiatry and Psychotherapy, University Medical Center Göttingen; Department of Neuroscience, University of Pittsburgh, USA

\section{Prof. Dr. André Fischer}

German Center for Neurodegenerative Diseases (DZNE) Göttingen;

Department of Psychiatry and Psychotherapy, University Medical Center Göttingen

\section{Prof. Dr. Dr. Hannelore Ehrenreich}

Department of Clinical Neuroscience, Max-Planck-Institute of Experimental Medicine Göttingen

\section{Members of the Examination Board}

\section{Referee: Dr. Dr. Oliver M. Schlüter}

Department of Psychiatry and Psychotherapy, University Medical Center Göttingen;

Department of Neuroscience, University of Pittsburgh, USA

\section{$2^{\text {nd }}$ Referee: Prof. Dr. André Fischer}

German Center for Neurodegenerative Diseases (DZNE) Göttingen;

Department of Psychiatry and Psychotherapy, University Medical Center Göttingen

\section{Further members of the Examination Board}

Prof. Dr. Dr. Hannelore Ehrenreich

Department of Clinical Neuroscience, Max-Planck-Institute of Experimental Medicine Göttingen

\section{Prof. Dr. Nils Brose}

Department of Molecular Neurobiology, Max-Planck-Institute of Experimental Medicine Göttingen

\section{Prof. Dr. Siegrid Löwel}

Department of Systems Neuroscience, Johann-Friedrich-Blumenbach-Institute of Zoology and Anthropology, University of Göttingen

\section{Prof. Dr. Michael Müller}

Department of Neuro- and Sensory Physiology, University Medical Center Göttingen 
I hereby declare that I have written the thesis entitled "A cortical hierarchy for differential GABAergic circuit motifs as targets for stress-induced alterations in endocannabinoid signaling" independently and with no other sources and aids than quoted.

Karl-Alexander Engelhardt

Göttingen, September 2020 


\section{TABLE OF CONTENTS}

ABSTRACT

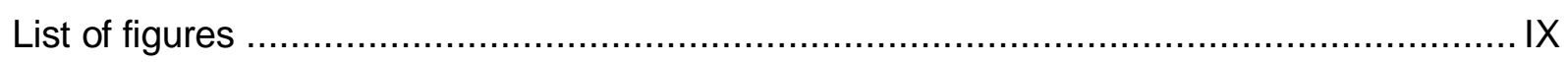

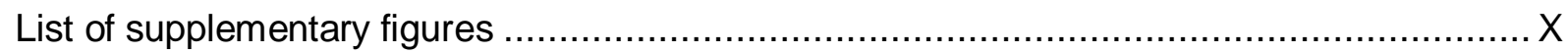

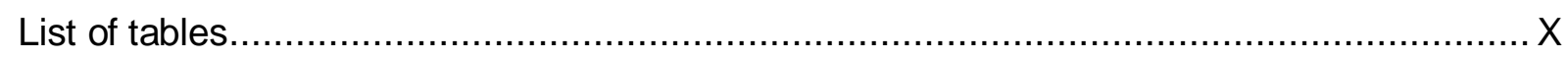

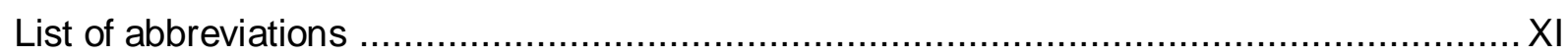

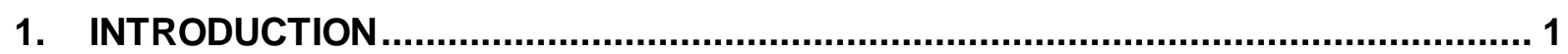

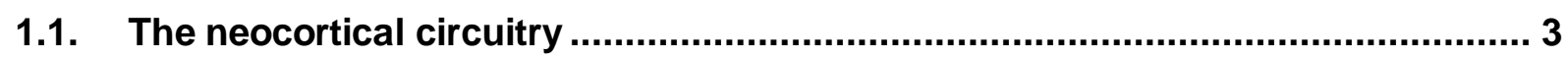

1.1.1. General anatomy and organizing principles................................................... 3

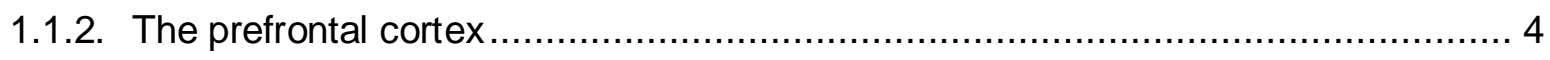

1.2. Synaptic communication ........................................................................ 5

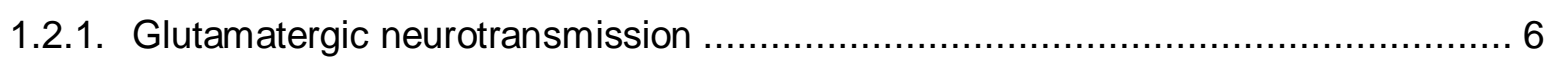

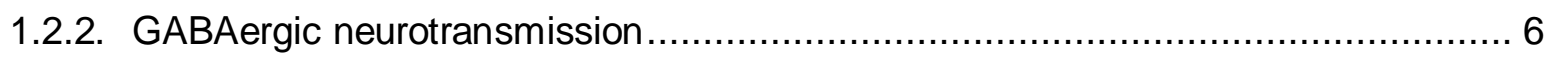

1.3. Endocannabinoid signaling ............................................................................ 8

1.3.1. Endocannabinoid-mediated plasticity at GABAergic synapses .......................... 9

1.3.2. Endocannabinoid-sensitive and -insensitive neocortical GABAergic circuits..........10

1.3.2.1. Endocannabinoid-insensitive parvalbumin-expressing interneurons ...............11

1.3.2.2. Endocannabinoid-sensitive cholecystokinin-expressing interneurons ............12

1.3.3. Role of endocannabinoid signaling in stress regulation ..................................13

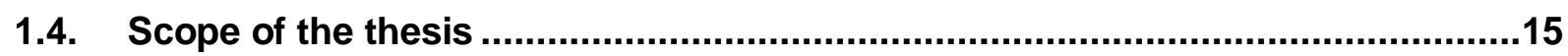

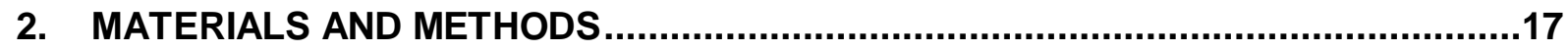

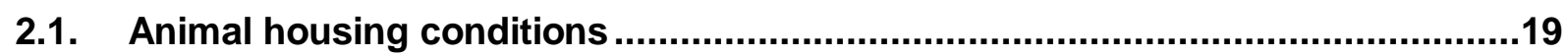

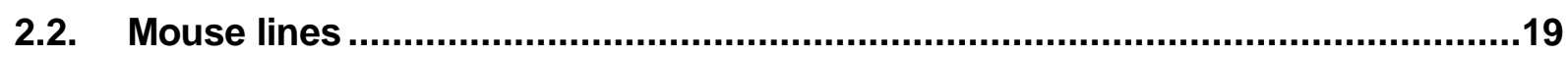

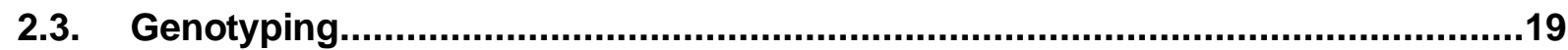

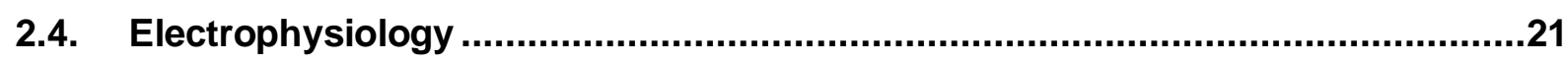




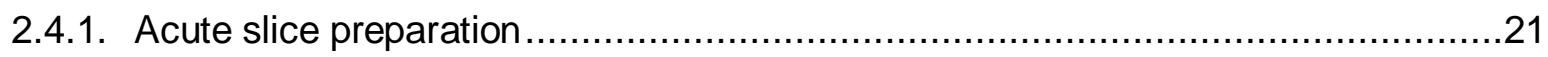

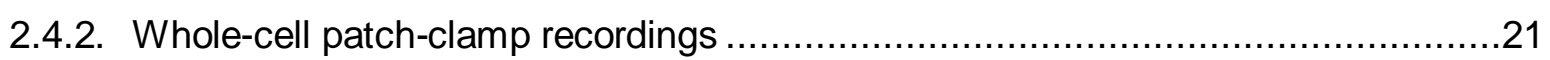

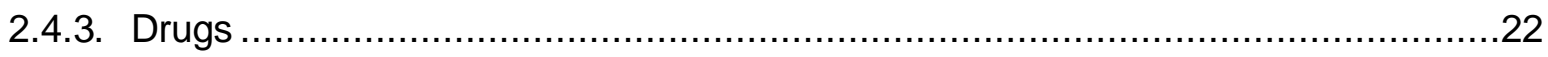

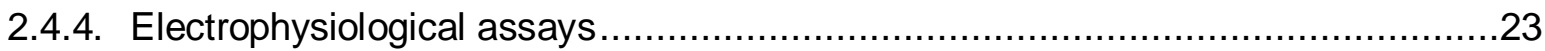

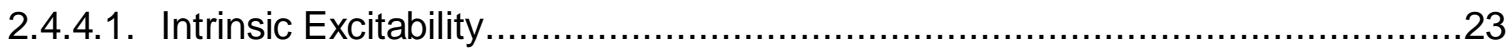

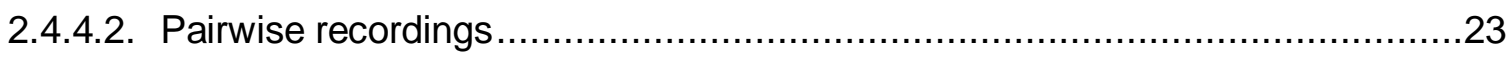

2.4.4.3. Depolarization-induced suppression of synaptic inhibition ...........................24

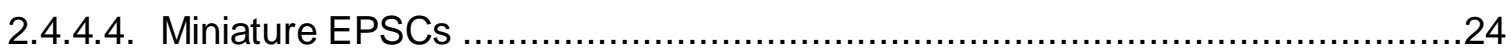

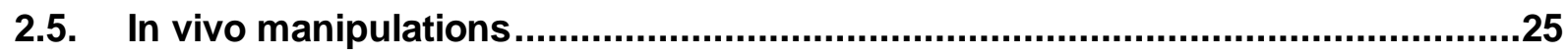

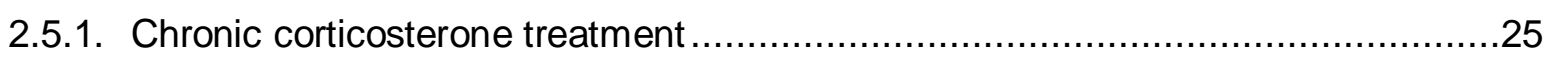

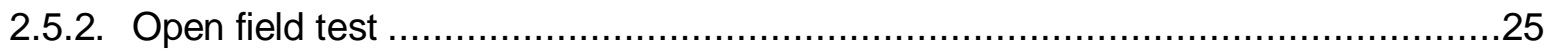

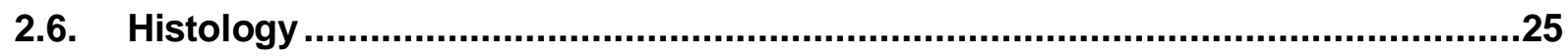

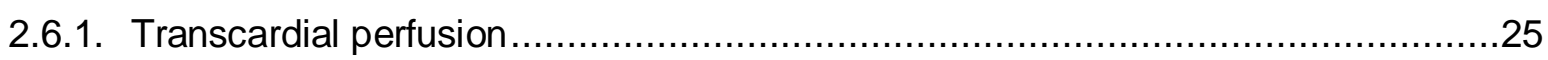

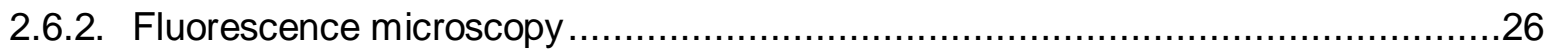

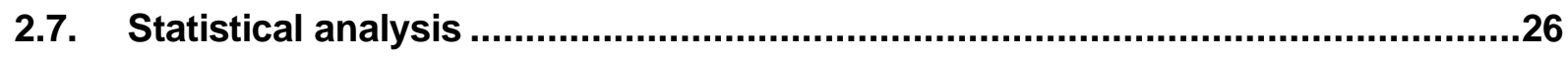

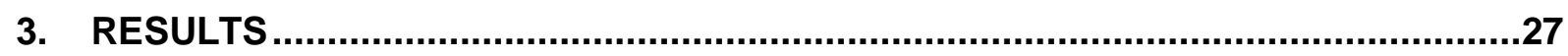

3.1. CB1+ and PV+ interneurons represent distinct cell types in neocortex.............29

3.2. The distribution of $\mathrm{CB1}+$ and $\mathrm{PV}+$ interneurons varies across cortical regions

3.3. The number and properties of GABAergic synapses formed by CB1+ and PV+ interneurons vary across cortical regions ..................................................32

3.3.1. CB1+ and PV+ interneurons form GABAergic synapses .............................32

3.3.2. GABAergic synapses made by $\mathrm{CB} 1+$ but not $\mathrm{PV}+$ interneurons are sensitive to retrograde endocannabinoid signaling

3.3.3. The inhibitory connectivity of $\mathrm{CB} 1+$ and $\mathrm{PV}+$ interneurons varies between

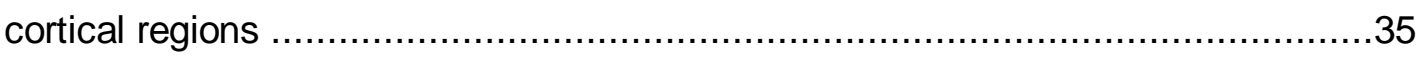

3.3.4. CB1+ interneurons form region-specific subtypes of GABAergic synapses ..........37 
3.4. Prefrontal inhibitory networks formed by $\mathrm{CB} 1+$ and $\mathrm{PV}+$ interneurons reach functional maturity early in development

3.4.1. GABAergic synapses of prefrontal $C B 1+$ and PV+ interneurons are formed early in development

3.4.2. Glutamatergic inputs onto prefrontal $P V+$ but not $C B 1+$ interneurons undergo synaptic remodeling during development

3.5. Prefrontal inhibitory networks formed by $\mathrm{CB} 1+$ but not PV+ interneurons show specific changes in response to developmental stress

3.5.1. Chronic corticosterone treatment induces a stress-related phenotype 44

3.5.2. Chronic corticosterone treatment does not affect GABAergic synapses made by $\mathrm{PV}+$ interneurons in prefrontal cortex

3.5.3. Chronic corticosterone treatment disrupts endocannabinoid signaling at GABAergic synapses made by CB1+ interneurons in prefrontal cortex

4. DISCUSSION

4.1. Summary of main findings.

4.2. CB1+ and PV+ interneurons represent anatomically and physiologically distinct cell types in neocortex

4.2.1. CB1+ interneurons display a high intrinsic excitability but receive only weak excitatory glutamatergic inputs compared to $\mathrm{PV}+$ interneurons

4.2.2. CB1+ interneurons form less reliable and precise GABAergic synapses compared to $\mathrm{PV}+$ interneurons

4.3. Cortical hierarchy shapes inhibitory network motifs formed by CB1+ and PV+ interneurons

4.3.1. Inhibitory control is dominated by $\mathrm{PV}+$ interneurons in primary somatosensory cortex

4.3.2. Inhibitory control is balanced between $\mathrm{PV}+$ and $\mathrm{CB} 1+$ interneurons in prefrontal cortex

4.3.3. CB1+ interneurons form distinct subtypes of GABAergic synapses in primary somatosensory but not prefrontal cortex 
4.4. Stress-related glucocorticoid signaling during adolescence induces selective alterations in endocannabinoid signaling at synapses made by CB1+ interneurons in prefrontal cortex

4.4.1. Inhibitory networks made by prefrontal PV+ and CB1+ interneurons mature early during development

4.4.2. Chronic corticosterone treatment during adolescence does not affect basal synaptic transmission at GABAergic synapses made by prefrontal $\mathrm{PV}+$ and CB1+ interneurons.

4.4.3. Chronic corticosterone treatment during adolescence impairs endocannabinoidmediated retrograde control of synaptic inhibition by prefrontal CB1+ interneurons

4.5. Conclusions and Outlook

5. REFERENCES

6. APPENDIX

6.1. Supplementary Figures.

6.2. List of chemicals and reagents .89

6.3. List of drugs

7. ACKNOWLEDGEMENTS. 


\section{Abstract}

Aberrant endocannabinoid ( $\mathrm{eCB}$ ) signaling has been implicated in the pathophysiology of different stress-related psychiatric diseases, such as schizophrenia, depression, and anxiety disorders. Such eCB signaling is triggered in postsynaptic neurons and serves to transiently suppress presynaptic neurotransmitter release by activating presynaptic cannabinoid type-1 (CB1) receptors. CB1 receptors are most abundantly expressed in a subpopulation of cortical GABAergic interneurons (INs), which serve to fine-tune cortical information flow by exerting inhibitory control over excitatory networks. Thus, CB1 receptor-expressing (CB1+) INs may represent a substrate linking changes in eCB signaling with stress-induced disease states. However, their function is only poorly understood.

I therefore characterized the properties of $\mathrm{CB} 1+\mathrm{INs}$ in neocortex and compared them with parvalbumin-expressing (PV+) INs, a well-characterized IN type with an established role in network refinement. To this end, I used a combination of fluorescent imaging, patch-clamp electrophysiology, and pharmacology in double reporter mice, in which CB1+ and PV+ INs are genetically labeled with tdTomato and YFP, respectively. I found that cortical hierarchy strongly shaped the expression of inhibitory circuit motifs made by the two IN types. Specifically, CB1+ INs were considerably less abundant and made considerably fewer GABAergic synapses onto glutamatergic pyramidal neurons compared with PV+ INs in the primary somatosensory cortex, a representative cortical region for primary sensory processing. In contrast, the abundance and inhibitory connectivity was largely balanced between the two IN types in the prefrontal cortex, a higher-order associative cortical structure that serves an important function in cognitive control and stress regulation.

I further characterized the inhibitory circuit properties of the two IN types in the prefrontal cortex across development and assessed their vulnerability towards glucocorticoid-mediated developmental stress. To this end, mice were chronically treated with the stress hormone corticosterone during adolescence, a developmental period of heightened stress vulnerability. I found that GABAergic synapses made by the two IN types onto prefrontal pyramidal neurons reached functional maturity early, before the onset of the adolescent phase. Only their glutamatergic inputs seemed to undergo some form of synapse pruning during adolescence, but these developmental changes were mostly restricted to PV+ INs. Remarkably, GABAergic synapses made by both prefrontal PV+ and CB1+ INs were highly resistant to chronic corticosterone treatment during adolescence, despite inducing a prominent stress- and anxiety-related phenotype. Indeed, basal synaptic transmission was completely preserved at both types of synapses in mice chronically treated with corticosterone. However, GABAergic synapses made by CB1+ INs displayed a deficiency in depolarization-induced suppression of synaptic inhibition, an eCB-dependent form of short-term synaptic plasticity. These results thus 
indicate a specific corticosterone-induced deficit in eCB-mediated regulation of synaptic transmission at these synapses.

Together, these findings suggest an important role of cortical hierarchy in shaping inhibitory circuit motifs and point to an increased importance of CB1+ INs in regulating excitatory network activity in higher-order cortical structures, such as the prefrontal cortex. Moreover, my results highlight a specific dysfunction in eCB-dependent plasticity at synapses of prefrontal CB1+ INs following chronic glucocorticoid exposure. Thus, CB1+ INs might represent a promising new target for the study of stress-related psychiatric disorders. Future studies should determine to what extent the observed deficit in eCB-dependent plasticity is causally related to stressinduced disease states.

Keywords: Endocannabinoid, cannabinoid type-1 receptor, parvalbumin, interneuron, GABAergic synapse, prefrontal cortex, stress, corticosterone 


\section{List of figures}

Figure 1: PV+ and CB1+ interneurons represent functionally distinct cell types in prefrontal cortex

Figure 2: The abundance of PV+ and CB1+ interneurons converges from somatosensory towards prefrontal cortex

Figure 3: $\mathrm{PV}+$ and $\mathrm{CB} 1+$ interneurons form GABAergic synapses onto neocortical pyramidal neurons

Figure 4: $\mathrm{CB} 1+$ but not $\mathrm{PV}+$ interneurons exhibit endocannabinoid-dependent depolarization-induced suppression of synaptic inhibition

Figure 5: The inhibitory connectivity of $\mathrm{PV}+$ and $\mathrm{CB} 1+$ interneurons converges from somatosensory to prefrontal cortex.

Figure 6: CB1+ interneurons form distinct subtypes of GABAergic synapses in somatosensory but not prefrontal cortex

Figure 7: $\mathrm{CB} 1+$ and $\mathrm{PV}+$ interneurons form functional GABAergic synapses onto pyramidal neurons in prefrontal cortex before the adolescent phase.

Figure 8: Developmental changes in mEPSCs during adolescence are more prominent in

$\mathrm{PV}+$ than in $\mathrm{CB} 1+$ interneurons in prefrontal cortex

Figure 9: Chronic corticosterone treatment induces stress-related physiological and behavioral changes.

Figure 10: Chronic corticosterone treatment does not affect synaptic transmission at GABAergic synapses made by $\mathrm{PV}+$ interneurons in prefrontal cortex

Figure 11: Chronic corticosterone treatment affects depolarization-induced suppression of synaptic inhibition but not basal synaptic transmission at GABAergic synapses made by $\mathrm{CB} 1+$ interneurons in prefrontal cortex

Figure 12: A cortical hierarchy for endocannabinoid-regulated stress-sensitive inhibitory circuits in neocortex 


\section{List of supplementary figures}

Supplementary Figure S1: The neutral CB1 receptor-selective antagonist NESS0327 does not affect basal synaptic strength and release properties at synapses made by $\mathrm{CB} 1+$ interneurons in prefrontal cortex

Supplementary Figure S2: PV+ and CB1+ interneurons form GABAergic synapses with different kinetic properties in somatosensory and prefrontal cortex

Supplementary Figure S3: Dimensionality reduction of the kinetic properties of GABAergic synapses made by CB1+ interneurons.

Supplementary Figure S4: Dimensionality reduction of the kinetic properties of GABAergic synapses made by PV+ interneurons

Supplementary Figure S5: PV+ interneurons do not form distinct subtypes of GABAergic synapses in either somatosensory or prefrontal cortex

Supplementary Figure S6: Synaptic properties of hierarchical cluster solutions obtained for synapses made by $\mathrm{CB} 1+$ and $\mathrm{PV}+$ interneurons in somatosensory and prefrontal cortex

Supplementary Figure 57: Cluster membership does not correlate with the firing properties of $\mathrm{CB} 1+$ interneurons in either somatosensory or prefrontal cortex.....

\section{List of tables}

Table 1: PCR conditions for genotyping of PV-Cre, iYFP, and CB1-tdTomato. 20

Table 2: Primer sequences and band sizes for PV-Cre, iYFP, and CB1-tdTomato 21 


\section{List of abbreviations}

$2-A G$

5-HT3a receptor

5-HT3a+

ACSF

AEA

AMPA

ANOVA

AP

art-ANOVA

cAMP

bp

BLA

CC

CCK

$\mathrm{CCK}_{+}$

CB1 receptor

$\mathrm{CB} 1_{\text {ir }}$

$\mathrm{CB} 1_{\text {rs }}$

CB1+

CB2 receptor

$\mathrm{Cg}$

CORT

DAGL

DMSO

DSI

eCB

E/l balance

EPSP

EPSC

$\mathrm{EtOH}$

$\mathrm{FAAH}$

fwd

GABA 2-arachidonoylglycerol

serotonin 3a receptor

serotonin 3a receptor-expressing

artificial cerebrospinal fluid

$\mathrm{N}$-arachidonoylethanolamine

a-amino-3-hydroxy-5-methyl-4-isoxazolepropionic acid

analysis of variance

action potential

aligned-rank transform analysis of variance

cyclic adenosine monophosphate

base pair

basolateral amygdala

current-clamp

cholecystokinin

cholecystokinin-expressing

cannabinoid type-1 receptor

irregular-spiking cannabinoid type-1 receptor-expressing interneuron

regular-spiking cannabinoid type-1 receptor-expressing interneuron

cannabinoid type-1 receptor-expressing

cannabinoid type-2 receptor

cingulate

corticosterone

diacylglycerol lipase

dimethyl sulfoxide

depolarization-induced suppression of synaptic inhibition

endocannabinoid

excitation/inhibition balance

excitatory postsynaptic potential

excitatory postsynaptic current

ethanol

fatty acid amide hydrolase

forward

gamma-aminobutyric acid 


\begin{tabular}{|c|c|}
\hline GAD & glutamic acid decarboxylase \\
\hline GAT & plasma membrane GABA reuptake transporter \\
\hline HPA axis & hypothalamus-pituitary-adrenal axis \\
\hline IL & infralimbic \\
\hline IN & interneuron \\
\hline IPSP & inhibitory postsynaptic potential \\
\hline IPSC & inhibitory postsynaptic current \\
\hline ir & immunoreactive \\
\hline$I-V$ curve & current-voltage curve \\
\hline iYFP & inducible yellow fluorescent protein \\
\hline $\mathrm{KI}$ & knock-in \\
\hline $\mathrm{L} 2 / 3$ & layer 2/3 \\
\hline L4 & layer 4 \\
\hline L5/6 & layer $5 / 6$ \\
\hline LTDi & long-term depression at inhibitory synapses \\
\hline MAGL & monoacylglycerol lipase \\
\hline mEPSC & miniature excitatory postsynaptic current \\
\hline mGlu receptor & metabotropic glutamate receptor \\
\hline mPFC & medial prefrontal cortex \\
\hline NAc & nucleus accumbens \\
\hline NAT & $\mathrm{N}$-acyl transferase \\
\hline NMDA & N-methyl D-aspartic acid \\
\hline NMDG & N-methyl-D-glucamine \\
\hline NOS & nitric oxide synthase \\
\hline $\mathrm{NOS}+$ & nitric oxide synthase-expressing \\
\hline$P$ & postnatal day \\
\hline PBS & phosphate-buffered saline \\
\hline PC & principal component \\
\hline PCR & polymerase chain reaction \\
\hline PFA & paraformaldehyde \\
\hline PLC & phospholipase C \\
\hline PLD & phospholipase D \\
\hline PKA & protein kinase $A$ \\
\hline PPR & paired-pulse ratio \\
\hline PN & pyramidal neuron \\
\hline
\end{tabular}




$\begin{array}{ll}\text { Pr } & \text { presynaptic release probability } \\ \text { PrL } & \text { prelimbic } \\ \text { PTX } & \text { picrotoxin } \\ \text { PV } & \text { parvalbumin } \\ \text { PV+ } & \text { parvalbumin-expressing } \\ \text { rev } & \text { reverse } \\ \text { rm-ANOVA } & \text { repeated-measures analysis of variance } \\ \text { RMP } & \text { resting membrane potential } \\ \text { rpm } & \text { revolutions per minute } \\ \text { SEM } & \text { standard error of the mean } \\ \text { SIPSC } & \text { spontaneous inhibitory postsynaptic current } \\ \text { SST } & \text { somatostatin } \\ \text { SST+ } & \text { somatostatin-expressing } \\ \text { S1 } & \text { primary somatosensory cortex } \\ \text { TTX } & \text { tetrodotoxin } \\ \text { UIPSC } & \text { unitary inhibitory postsynaptic potential } \\ \text { VC } & \text { voltage-clamp } \\ \text { VEH } & \text { vehicle } \\ \text { VIP } & \text { vasoactive-intestinal peptide } \\ \text { VIP+ } & \text { vasoactive-intestinal peptide-expressing } \\ \text { V1 } & \text { voltage-gated Catow channel } \\ \text { VGCC } & \\ \text { WT } & \text { wildtype } \\ \text { YFP } & \text { yelloorescent protein } \\ & \end{array}$


1. Introduction 


\subsection{The neocortical circuitry}

The mammalian neocortex is a complex network of billions of functionally connected neurons that form specialized neuronal circuits. These neocortical circuits are thought to mediate most forms of higher-order neural information processing and give rise to the vast majority of our perceptions, thoughts, emotions, and behavior (Rakic, 2009).

Neocortical circuits are by no means hardwired or stereotyped in their function. On the contrary, they are dynamically regulated and prone to undergo various forms of experiencedependent circuit remodeling, thereby allowing us to adapt to the ever-changing demands of the environment. One of the most striking examples of such cortical circuit remodeling is the potential for topographical reorganization of cortical maps following brain injury through rehabilitative training where, under certain conditions, neighboring regions can even take over the function of lesioned tissue to enable functional recovery (e.g. Nudo et al., 1996; for review see: Xerri, 2012). However, this high degree of plasticity also confers vulnerability towards environmental insult, e.g. psychosocial stress, particularly during critical periods in postnatal development, possibly leading to long-lasting neural circuit dysfunction and increasing the risk of developing psychiatric disorders (Caspi et al., 2003; Andersen et al., 2008; Marco et al., 2011). Understanding the cellular substrates and operating principles of neocortical network function, as well as their disruption by stress, may thus ultimately help us gain deeper insights into the pathophysiology of psychiatric diseases.

\subsubsection{General anatomy and organizing principles}

The neocortex is a phylogenetically young structure that emerged during the evolution of mammalian species (Rakic, 2009). Many species-specific differences can be found between neocortex of humans and rodents, including size, complexity, or the presence of convolutions. However, the basic circuit organization and functional anatomy are largely homologous (Douglas and Martin, 2004; Rakic, 2009; Harris and Shepherd, 2015).

The neocortex is a multilayered structure that is composed of six different layers. These layers are populated by distinct neuronal cell types and participate in a largely defined flow of cortical information processing (Douglas and Martin, 2004). Sensory information is conveyed from our sensory organs to different thalamic nuclei and then further relayed into different parts of the neocortex. In a basic circuit model originally developed by Gilbert and Wiesel (1979), thalamic inputs mainly reach excitatory projection neurons in the thalamocortical recipient layer 4 (L4). L4 neurons in turn forward the information to so-called pyramidal neurons in supragranular input layers $L 2 / 3$. The supragranular layers L2/3 are considered the main input source of the neocortical circuitry, where information is further processed, integrated with inputs from other cortical and subcortical regions, and forwarded to other cortical structures in order to enable signal processing in parallel, functionally segregated information streams. L2/3 pyramidal neurons in turn transfer the information to pyramidal neurons in infragranular output layers 
L5/6, which project to subcortical structures including the basal ganglia, brainstem nuclei, and spinal cord to generate a behavioral output. These neurons additionally have recurrent feedback projections to layers $L 4$ and $L 2 / 3$ and also project to thalamic relay nuclei to adjust the ongoing cortical information flow. At all neocortical layers, information processing is finetuned by locally acting inhibitory neurons (for review see: Douglas and Martin, 2004; Harris and Shepherd, 2015; Shepherd and Rowe, 2017).

A central feature of the neocortex is its hierarchical organization (Harris and Shepherd, 2015). In a simplified scheme of bottom-up processing, different types of sensory input are first processed in primary sensory cortices, such as visual inputs in the primary visual cortex (V1) and somatosensory inputs in primary somatosensory cortex (S1). From there, information is usually forwarded to secondary sensory cortices and finally to higher-order parietal and frontal association centers, which serve to integrate inputs from different sensory modalities and perform higher-order cortical functions, such as executive control. In addition, higher-order cortical regions send feedback projections to lower-level structures for top-down modulation of sensory processing, such as filtering task-irrelevant information via selective attention (Gilbert and Li, 2013; Gregoriou et al., 2014). The basic circuit motifs are largely conserved between these regions (Douglas and Martin, 2004; Harris and Shepherd, 2015). However, several variations in anatomy and circuit properties can be found between the different neocortical areas (Harris and Shepherd, 2015).

\subsubsection{The prefrontal cortex}

The prefrontal cortex is the most prominent higher-order cortical structure. It serves as a major association center that integrates various sources of information in order to guide different forms of goal-directed behavior (Miller, 2000; Brown and Bowman, 2002; Arnsten, 2009). Indeed, the prefrontal cortex is strongly enlarged in primate species, including humans, and therefore believed to underlie our most unique human mental abilities (Rakic, 2009).

In rodents, the prefrontal cortex is commonly referred to as medial prefrontal cortex (mPFC). It is located at the medial wall of the frontal lobe and often subdivided into cingulate $(\mathrm{Cg})$, prelimbic (PrL), and infralimbic (IL) portions (Heidbreder and Groenewegen, 2003; Laubach et al., 2018). The anatomical homologies between rodent and primate prefrontal cortex are still a matter of active scientific debate, but many functional similarities exist (Brown and Bowman, 2002; Laubach et al., 2018). The rodent mPFC is anatomically distinguished from primary sensory regions by the absence of a distinctive thalamocortical recipient layer L4. However, it is heavily interconnected with the mediodorsal thalamus, particularly its input layers L2/3 (e.g. Collins et al., 2018; for a thorough review see: Heidbreder and Groenewegen, 2003). Moreover, the mPFC exhibits dense long-range reciprocal connectivity with the vast majority of neocortical regions and various subcortical and limbic structures, such as hypothalamus, amygdala, nucleus accumbens, and hippocampus (Miller, 2000; Heidbreder and 
Groenewegen, 2003). The mPFC is thus strategically positioned to integrate various converging neocortical and subcortical inputs and to exert hierarchical control over different lower-level targets to modulate brain-wide circuit activity during ongoing behavior (Miller, 2000). These properties enable the prefrontal cortex to govern and maintain various higherorder cognitive processes, such as executive control, working memory, behavioral flexibility, abstract reasoning, social behavior, and mood regulation (Miller, 2000; Brown and Bowman, 2002; Heidbreder and Groenewegen, 2003).

The prefrontal cortex undergoes a protracted phase of circuit remodeling during postnatal development in order to reach its functional maturity (Huttenlocher and Dabholkar, 1997; Petanjek et al., 2011). This protracted development spans throughout adolescence, the presumed critical developmental period for higher-order cognitive abilities, and is thought to render the prefrontal cortex particularly vulnerable to environmental insult (Gee and Casey, 2015; Caballero and Tseng, 2016). Indeed, the prefrontal cortex is highly stress-susceptible compared to other cortical regions (Arnsten, 2009). This vulnerability is especially pronounced during adolescence and has been implicated in the etiology of various psychiatric disorders, including schizophrenia, major depression, and anxiety disorders (Arnsten, 2009; Caballero and Tseng, 2016).

\subsection{Synaptic communication}

Synapses are specialized structures that enable electrochemical signaling and thus information transfer between neurons. Two main synapse classes can be distinguished: electrical and chemical synapses.

Electrical synapses are established through membrane-associated ion channel pores, which directly connect the intracellular milieu of two neurons. They are commonly referred to as gap junctions and allow rapid, bidirectional current flow between neurons through direct intercellular diffusion of ions and other molecules (Jessell and Kandel, 1993; Alcamí and Pereda, 2019). Electrical coupling helps synchronize neuronal networks, which is assumed to improve neural signal processing (Tamás et al., 2000; Alcamí and Pereda, 2019).

Chemical synapses represent the majority of synapses in the mammalian brain and use the additional release of a neurotransmitter for signal transduction (Jessell and Kandel, 1993). They consist of a pre- and a postsynaptic site, which are separated by a thin gap called the synaptic cleft. Activation of a presynaptic neuron generates an action potential (AP), which travels along the axon, depolarizes the presynaptic terminal, and triggers the opening of voltage-gated $\mathrm{Ca}^{2+}$ channels (VGCCs). The resulting $\mathrm{Ca}^{2+}$ influx causes presynaptic vesicles, containing neurotransmitter molecules, to fuse with the presynaptic membrane and to release their content into the synaptic cleft. The released neurotransmitters bind to ligand-gated ion channel receptors on the postsynaptic membrane, which convert the chemical back to an electrical signal (Jessell and Kandel, 1993; Sabatini and Regehr, 1999). Chemical synapses 
come at a remarkable molecular and functional diversity (Nusser, 2018). They can be broadly divided into two distinct types formed by two main classes of neurons: excitatory synapses, which are formed by glutamatergic principal neurons, and inhibitory synapses, which are formed by gamma-aminobutyric acid (GABA)ergic neurons.

\subsubsection{Glutamatergic neurotransmission}

Neural information processing is largely mediated by excitatory activity generated within networks of synaptically connected principal neurons. Principal neurons are the most abundant cell type in the cerebral cortex and usually distinguished by their pyramidal-like morphology, extensive dendritic arborization, and long-range projecting axons that innervate different regions of the brain. They are therefore often referred to as pyramidal projection neurons. These neurons mediate the majority of information transfer between different neocortical layers and regions (Harris and Shepherd, 2015; Shepherd and Rowe, 2017).

Principal neurons enable signal propagation by forming excitatory synapses onto their target neurons. These excitatory synapses are usually located on small protrusions along the dendrites of postsynaptic principal neurons called dendritic spines and release the neurotransmitter glutamate (Yuste and Denk, 1995). Synaptically released glutamate activates postsynaptic glutamate receptors, most notably a-amino-3-hydroxy-5-methyl-4isoxazolepropionic acid (AMPA) and N-methyl-D-aspartic acid (NMDA) receptors, which are ionotropic receptors with gated, cation-permeable ion channel pores. Receptor activation causes these channels to open with a net flow of cations (primarily $\mathrm{Na}^{+}$) into the postsynaptic cell, triggering a local membrane depolarization called excitatory postsynaptic potential (EPSP). These EPSPs can be electrotonically or actively conducted along the dendrite towards the soma, where they summate with other EPSPs to increase the probability of a postsynaptic neuron to fire APs. Such fast excitatory synaptic transmission is largely mediated by AMPA receptors. By contrast, NMDA receptors have slower conductance and only transmit at depolarized potentials (Stern et al., 1992; Scannevin and Huganir, 2000). Additionally, synaptic function at excitatory synapses can be permanently modulated by the activity of metabotropic glutamate (mGlu) receptors, which are G-protein-coupled receptors acting through secondmessenger pathways (Conn and Pin, 1997).

\subsubsection{GABAergic neurotransmission}

GABAergic neurons only make up $\sim 15 \%$ of the total neuronal population in neocortex (Meyer et al., 2011). However, they are an integral part of excitatory circuits and serve an important function in regulating cortical information flow by fine-tuning the activity of their target neurons (Freund and Katona, 2007; Rudy et al., 2011; Fino et al., 2013; Tremblay et al., 2016). GABAergic neurons are mostly locally-projecting and form synapses preferentially onto nearby neurons. They are therefore often referred to as interneurons (INs). 
GABAergic INs fine-tune cortical information flow by forming inhibitory synapses onto their target neurons. These synapses release the inhibitory neurotransmitter GABA, which activates $\mathrm{Cl}^{-}$-permeable ionotropic $\mathrm{GABA}_{A}$ receptors on postsynaptic sites. Their opening at resting potential typically results in an inward $\mathrm{Cl}^{-}$conductance, which hyperpolarizes the postsynaptic membrane and generates an inhibitory postsynaptic potential (IPSP), thereby decreasing the AP firing probability of postsynaptic neurons (Isaacson and Scanziani, 2011; Sigel and Steinmann, 2012). Activation of GABAergic synapses can also reduce cell excitability even without net current flow if the membrane potential is close to the $\mathrm{Cl}^{-}$reversal potential through so-called shunting (or silent) inhibition (Isaacson and Scanziani, 2011). Unlike hyperpolarizing inhibition, shunting inhibition is a form of non-linear or divisive inhibition, in which the mere opening of $\mathrm{GABA}_{A}$ receptors can short-cut EPSPS just by increasing the ionic leak conductance of postsynaptic cells. Such shunting inhibition is believed to have an important function in gain control by adjusting a neuron's sensitivity range towards synaptic inputs (Mitchell and Silver, 2003; Prescott and Koninck, 2003).

$\mathrm{GABA}_{A}$ receptors mediate the vast majority of phasic synaptic inhibition in neocortex (Farrant and Nusser, 2005). They are pentameric ion channel pores composed of different subunits. The major subunit families are the $\alpha, \beta$, and $\gamma$ subunits, each containing several family members. The most common subunit assembly of the $\mathrm{GABA}_{A}$ receptor is made up of two $\alpha 1$ subunits, two $\beta 2$ subunits, and one y2 subunit (Sigel and Steinmann, 2012). However, many other subunits can be found e.g. depending on subcellular localization. GABA $A_{A}$ receptors exhibit two GABA binding sites and an additional allosteric benzodiazepine binding site that modulates channel activity and is the site of action of a class of sedative and anxiolytic drugs called benzodiazepines (Sigel and Steinmann, 2012). They are anchored and clustered at postsynaptic sites through different scaffolding proteins, most notably gephyrin (Kneussel et al., 1999; Krueger-Burg et al., 2017). However, they can also be located at extrasynaptic sites, where they mediate tonic inhibition (Stell et al., 2003). Inhibitory synapses further express metabotropic $\mathrm{GABA}_{B}$ receptors. Postsynaptically, these receptors mediate slowly conducting inhibitory responses through activation of inwardly rectifying $\mathrm{K}^{+}$channels. Moreover, they can serve as autoreceptors on presynaptic sites to block transmitter release during excess presynaptic activity (Isaacson and Scanziani, 2011).

GABA is synthesized from glutamate at presynaptic sites by the glutamic acid decarboxylases GAD67 and GAD65 and is further packaged into synaptic vesicles via the vesicular GABA transporter (Jin et al., 2003). Synaptic clearance is carried out by different plasma membrane GABA reuptake transporters (GATs). In neocortex, reuptake is mostly mediated via GAT1 (Keros and Hablitz, 2005). 


\subsection{Endocannabinoid signaling}

The endocannabinoid (eCB) system is an evolutionarily old neuromodulator system in vertebrate species that can already be found in amphibians (Soderstrom et al., 2000). eCBs act as retrograde messengers, which distinguishes them from most other neurotransmitter systems (Hashimotodani et al., 2007). They are released from postsynaptic neurons in a retrograde manner and function to suppress presynaptic neurotransmitter release by activating endogenous cannabinoid receptors on presynaptic nerve terminals. Such eCBs are considered powerful modulators of synaptic function and mediate various forms of short- and long-term synaptic plasticity at both excitatory and inhibitory synapses (Pitler and Alger, 1992; 1994; Kreitzer and Regehr, 2001; Wilson and Nicoll, 2001; Ohno-Shosaku et al., 2001; Gerdeman et al., 2002; Chevaleyre and Castillo, 2003).

eCBs are lipophilic molecules that are synthesized from phospholipid precursors. Two main types of eCBs exist in the brain: $\mathrm{N}$-arachidonoylethanolamine (AEA, or "anandamide") and 2arachidonoylglycerol (2-AG). They are produced and degraded through different metabolic pathways (Hashimotodani et al., 2007; Kano et al., 2009). AEA is predominantly synthesized from phosphatidylethanolamine in different bioenzymatic steps by $\mathrm{N}$-acyl transferase (NAT) and phospholipase D (PLD). AEA is in turn degraded by fatty acid amide hydrolase (FAAH), which is the major AEA-degrading enzyme in the brain and is mostly found at the site of production in neuron somata and dendrites (Egertová et al., 1998; 2003; Gulyas et al., 2004). By contrast, 2-AG is mostly synthesized from phosphatidylinositol by phospholipase C (PLC) and diacylglycerol lipase (DAGL). 2-AG is then primarily catabolized by the degrading enzyme monoacylglycerol lipase (MAGL). In contrast to FAAH, MAGL is present in presynaptic neuron terminals as well as astrocytes (Gulyas et al., 2004; Uchigashima et al., 2011). It has been proposed that synaptically released 2-AG first enters the presynaptic phospholipid bilayer and in turn activates CB1 receptors via lateral diffusion. Such lipid-bound 2-AG can be degraded by MAGL either before or after reaching CB1 receptors (Kano et al., 2009). AEA and 2-AG are not stored in synaptic vesicles and are instead synthesized and released on demand from postsynaptic neurons in an activity-dependent manner (e.g. Wilson and Nicoll, 2001; Kreitzer and Regehr, 2001; Chevaleyre and Castillo, 2003; 2004; for review see: Hashimotodani et al., 2007; Kano et al., 2009; Castillo et al., 2012).

Retrograde eCB signaling is mediated through two main types of endogenous $\mathrm{G}_{\mathrm{i} / 0^{-}}$-coupled metabotropic cannabinoid receptors: cannabinoid type-1 (CB1) receptors, which are predominantly expressed in neurons, and cannabinoid type-2 (CB2) receptors, which are mainly found in immune cells, such as microglia (Kano et al., 2009). CB1 receptors mediate the vast majority of synaptic effects produced by retrograde eCB signaling (Hashimotodani et al., 2007; Kano et al., 2009; Castillo et al., 2012). They are mostly located on presynaptic terminals (Katona et al., 1999; 2006). Activation of CB1 receptors leads to G protein-dependent 
inhibition of presynaptic VGCCs, thereby blocking AP-dependent $\mathrm{Ca}^{2+}$ influx and thus neurotransmitter release (Mackie and Hille, 1992; Wilson et al., 2001; Kreitzer and Regehr, 2001). However, their activation can also have a long-lasting effect on the presynaptic release machinery by modulating cyclic adenosine monophosphate/protein kinase A (cAMP/PKA) signaling (Chevaleyre et al., 2007)

\subsubsection{Endocannabinoid-mediated plasticity at GABAergic synapses}

CB1 receptors are located on both glutamatergic and GABAergic axon terminals. However, they are most abundantly expressed at GABAergic synapses (Marsicano and Lutz, 1999; Hill et al., 2007; Steindel et al., 2013). This has been convincingly demonstrated in studies using cell type-specific knockout of CB1 receptors. Indeed, conditional deletion of CB1 receptors from GABAergic neurons leads to a profound loss of CB1 receptor immunoreactivity and protein levels in the hippocampus (Steindel et al., 2013). In contrast, immunoreactivity and protein levels are largely preserved following conditional deletion of CB1 receptors from glutamatergic projection neurons, suggesting that the majority of functional CB1 receptors is localized on GABAergic terminals (Steindel et al., 2013). At these GABAergic synapses, CB1 receptors function to down-regulate inhibitory presynaptic inputs following phasic retrograde eCB signaling (Wilson and Nicoll, 2001; Ohno-Shosaku et al., 2001). Such phasic eCB signaling seems to be largely mediated by the synthesis and release of 2-AG, which is triggered in postsynaptic pyramidal neurons during increased neuronal activity and serves to mediate different forms of short- and long-term inhibitory synaptic plasticity (Chevaleyre et al., 2006; Kano et al., 2009; Castillo et al., 2012).

A well-studied example of eCB-dependent inhibitory plasticity is called depolarization-induced suppression of synaptic inhibition (DSI), a form of short-term synaptic depression triggered by depolarization-induced postsynaptic $\mathrm{Ca}^{2+}$ signaling. During DSI, $\mathrm{Ca}^{2+}$ influx following short postsynaptic depolarization stimulates DAGL-mediated 2-AG signaling in pyramidal neurons. Retrograde 2-AG signaling then transiently suppresses GABA release at inhibitory synapses by activating presynaptic $\mathrm{CB} 1$ receptors, resulting in a $\mathrm{G}$ protein-dependent inhibition of VGCCs and thus decreased presynaptic, AP-dependent $\mathrm{Ca}^{2+}$ influx (Wilson and Nicoll, 2001; Ohno-Shosaku et al., 2001; Wilson et al., 2001; Gao et al., 2010; Tanimura et al., 2010; but see: Chevaleyre and Castillo, 2003). Such CB1 receptor-expressing GABAergic synapses can also undergo a long-term form of eCB-dependent plasticity called long-term depression at inhibitory synapses (LTDi). LTDi is triggered by activation of postsynaptic group I mGlu receptors following repetitive stimulation of excitatory glutamatergic synapses, which stimulates PLC and subsequently DAGL-mediated 2-AG signaling. This 2-AG signaling activates $\mathrm{CB} 1$ receptors at adjacent inhibitory synapses, thereby leading to a lasting reduction in GABA release (Chevaleyre and Castillo, 2003, 2004). 
DSI and LTDi can be considered different forms of heterosynaptic metaplasticity, which can gate excitatory activity in postsynaptic pyramidal neurons through disinhibition (Chevaleyre and Castillo, 2003; 2004; Fortin et al., 2004). For instance, pyramidal neuron excitability is enhanced during DSI (Fortin et al., 2004). Such eCB-dependent disinhibitory gating has also been demonstrated for long-term potentiation (LTP) at glutamatergic excitatory synapses in pyramidal neurons, which is facilitated by the induction of eCB-dependent LTDi (Chevaleyre and Castillo, 2004). Indeed, LTP is severely impaired in mice with a conditional deletion of CB1 receptors from GABAergic forebrain neurons, which thus generally lack the ability to undergo eCB-dependent inhibitory synaptic plasticity (Monory et al., 2015). Hence, retrograde eCB signaling serves an important function in synaptic crosstalk between excitatory and inhibitory synapses by detecting changes in the overall activity of pyramidal neurons to adjust the strength of GABAergic inputs. By these means, eCB signaling can powerfully modulate cortical circuit excitability (Hájos et al., 2000; Chevaleyre and Castillo, 2003; 2004; Fortin et al., 2004; Monory et al., 2015).

\subsubsection{Endocannabinoid-sensitive and -insensitive neocortical GABAergic circuits}

CB1 receptors seem to be targeted to specific IN subpopulations. Indeed, neocortical INs are highly diverse and many different subtypes can be distinguished based on various features, such as their morphology, firing properties, molecular expression patterns, and connectivity motifs (Rudy et al., 2011; Kepecs and Fishell, 2014; Tremblay et al., 2016). The nomenclature and classification systems are still debated. However, three main cardinal classes are currently distinguished: parvalbumin-expressing (PV+) INs, somatostatin-expressing (SST+) INs, and serotonin receptor 3a-expressing (5-HT3a+) INs (Rudy et al., 2011; Tremblay et al., 2016). Combined, these three cardinal IN types represent almost $100 \%$ of the total IN population in neocortex (Lee et al., 2010a). They exhibit distinct physiological properties and serve different functions in the regulation of neocortical circuit activity (Rudy et al., 2011; Tremblay et al., 2016). PV+ INs form dense GABAergic synapses onto the perisomatic region of pyramidal neurons, allowing them to control the firing output and AP timing of their target neurons and thereby enabling the generation of synchronous network activity (Sohal et al., 2009; Tremblay et al., 2016). By contrast, SST+ INs innervate the distal dendrites of pyramidal neurons (Nigro et al., 2018). They are therefore able to control the synaptic inputs of their target neurons and thus regulate local synaptic integration (Lovett-Barron et al., 2012). However, SST+ INs have also been shown to innervate different GABAergic IN types in addition to pyramidal neurons (Pfeffer et al., 2013). 5-HT3a+ INs are a highly heterogeneous IN category that can be further divided into numerous subtypes (Lee et al., 2010a). A major subtype are the so-called vasoactive-intestinal peptide-expressing (VIP+) INs (Lee et al., 2010a). These INs preferentially innervate other IN types rather than pyramidal neurons, particularly SST+ INs (Pfeffer et al., 2013; Lee et al., 2013). Therefore, VIP+ INs are more specialized in disinhibitory 
control by inhibiting other IN types, thereby releasing the inhibitory brake onto glutamatergic pyramidal neurons (Lee et al., 2013). CB1 receptor-expressing (CB1+) INs seem to represent a rather broad subcategory of 5-HT3a+ INs and could so far only be studied indirectly due to the lack of reporter lines to directly target these neurons. However, they considerably overlap with another subtype of mostly perisoma-targeting, cholecystokinin-expressing (CCK+) INs (Freund, 2003; Freund and Katona, 2007).

\subsubsection{Endocannabinoid-insensitive parvalbumin-expressing interneurons}

$\mathrm{PV}+$ INs represent the probably best characterized IN type in the cerebral cortex and have received considerable attention over the past decades (Tremblay et al., 2016). They express the calcium-binding protein PV and can be readily distinguished from other IN types by their fast-spiking firing properties, which allow them to generate APs at very high firing rates and virtually no frequency adaptation (e.g. Galarreta and Hestrin, 1999).

Fast-spiking PV+ INs in neocortex and hippocampus are negative for CB1 receptors and thus insensitive to retrograde eCB signaling (Marsicano and Lutz, 1999; Katona et al., 1999; Wilson et al., 2001; Bodor et al., 2005; Galarreta et al., 2008; but see: Jiang et al., 2010a). However, a special case exists in the nucleus accumbens (NAc). Indeed, we previously showed that a large fraction of $\mathrm{PV}+\mathrm{INs}$ expresses functional $\mathrm{CB} 1$ receptors in NAc, indicating a substantial overlap of PV+ and CB1+ INs in subcortical structures (Winters et al., 2012).

$\mathrm{PV}+$ INs form mostly a1 subunit-containing perisomatic GABAergic synapses onto pyramidal neurons (Freund, 2003). They can be divided into two main subtypes: basket cells, which form dense, basket-like synapses onto pyramidal somata and proximal dendrites, and chandelier cells, which exclusively target the axon initial segment of pyramidal neurons (Tremblay et al., 2016). Thus, GABAergic synapses made by PV+ INs are strategically positioned to control the output and temporal fidelity of AP firing in pyramidal neurons. Indeed, PV+ INs usually synapse onto a large territory of nearby pyramidal neurons (e.g. Packer and Yuste, 2011; Pfeffer et al., 2013). Additionally, they are electrically coupled to each other via gap junctions and receive strong and fast AMPA receptor-mediated excitatory inputs from glutamatergic projection neurons (Galarreta and Hestrin, 1999; Gabernet et al., 2005; Sun et al., 2006). All these properties enable PV+ INs to exert fast, synchronous, and remarkably precise feedforward inhibition over a large set of postsynaptic target neurons, creating narrow time "windows of opportunity" for their activation (Pinto et al., 2000; Tremblay et al., 2016). A well-studied example of feedforward inhibition exists in the thalamocortical L4 pathway in primary sensory regions, such as S1. Thalamic neurons innervate both L4 excitatory neurons and local PV+ INs. However, excitatory thalamocortical inputs onto PV+ INs are considerably stronger and faster, which readily drive them to fire and inhibit the activity of nearby $L 4$ excitatory neurons via their GABAergic output synapses (Gabernet et al., 2005; Sun et al., 2006). This strong and fast feedforward inhibition can only be overcome by synchronously timed thalamocortical 
inputs to rapidly trigger the firing of $L 4$ excitatory neurons before the onset of synaptic inhibition. These circuit properties thus favor the selection of synchronous, coincident activity over asynchronous, spontaneous activity (Pinto et al., 2000; Gabernet et al., 2005; Tremblay et al., 2016). PV+ INs are therefore often thought to act as coincidence detectors and pace makers in neocortical circuits that can synchronize the activity of large ensembles of pyramidal neurons to enhance the signal-to-noise ratio in a given network and thus improve neocortical information processing (e.g. Freund and Katona, 2007).

$\mathrm{PV}+$ INs are often considered the most abundant IN type in neocortex (Rudy et al., 2011; Fino et al., 2013; Tremblay et al., 2016). Indeed, they make up $40 \%$ of the total IN population (Lee et al., 2010a; Xu et al., 2010; Whissell et al., 2015) and have been associated with a wide range of cortical functions, including network synchrony, critical period plasticity, and excitation/inhibition balance (e.g. Sohal et al., 2009; Kuhlman et al., 2013; Xue et al., 2014). However, such findings are often derived from studies conducted at the primary sensory systems levels. Moreover, many other IN types can be found with specialized functions in the regulation of cortical network activity.

\subsubsection{Endocannabinoid-sensitive cholecystokinin-expressing interneurons}

Another important IN type are CCK+ INs. These neurons express the neuropeptide CCK and are considered a subtype of 5-HT3a+ INs (Tremblay et al., 2016). They usually have regularfiring or fast-accommodating/irregular-firing properties and thus generate APs with strongly adapting firing rates (Neu et al., 2007; Galarreta et al., 2008; Lee et al., 2010b).

$\mathrm{CCK}+\mathrm{INs}$ abundantly express the CB1 receptor on their presynaptic terminals and are thus often distinguished by a pronounced sensitivity towards retrograde eCB signaling (Marsicano and Lutz, 1999; Katona et al., 1999; Bodor et al., 2005). Indeed, they are sometimes interchangeably referred to as CCK/CB1+ INs and readily identified based on the presence of eCB-dependent modulation of their GABAergic output synapses (Földy et al., 2006; Neu et al., 2007; Lee et al., 2010b; 2015). However, there has been a paucity of studies on the function and properties of CCK+ INs in neocortex. This is presumably due to the rather non-selective expression of CCK in various cortical neuron types, including glutamatergic projection neurons (Hill et al., 2007), which limits the ability to selectively target CCK+ GABAergic neurons using conventional genetic labeling approaches, such as Cre recombinase driver lines (Taniguchi et al., 2011). CCK+ INs have been therefore mostly studied in the hippocampus, where they can be more readily localized based on additional criteria, such as their anatomical distribution (e.g. Neu et al., 2007; Földy et al., 2007; Lee et al., 2010b; 2011).

CCK+ INs mainly form a2 subunit-containing basket-like perisomatic GABAergic synapses onto pyramidal neurons (Freund, 2003; Freund and Katona, 2007). However, they can also form dendrite-targeting synapses depending on subtype, such as Schaffer collateralassociated CCK+ INs in hippocampus (Cope et al., 2002; Lee et al., 2010b). A prominent 
feature of inhibitory synapses made by $\mathrm{eCB}$-sensitive $\mathrm{CCK}+\mathrm{INs}$ is the rather unreliable, highly asynchronous mode of synaptic transmission. Specifically, these synapses often display only weak GABA release during single activation, but robust, largely asynchronous GABA release during repetitive stimulation. This asynchronous release persists even after the cessation of presynaptic activity, allowing CCK + INs to exert long-lasting synaptic inhibition over their target principal neurons (Losonczy et al., 2004; Hefft and Jonas, 2005). Moreover, CCK/CB1+ INs receive rather weak excitatory glutamatergic inputs, but are able to integrate these inputs over long time scales, as opposed to PV+ INs (Glickfeld and Scanziani, 2006). They further express receptors for different neuromodulators, such as acetylcholine and serotonin, and are therefore believed to be under strong neuromodulatory control by various subcortical afferents (Porter et al., 1999; Morales and Bäckman, 2002; Férézou et al., 2002; Morales et al., 2004; Cea-del Rio et al., 2010). Due to these properties, eCB-sensitive CCK+ INs have been suggested to integrate different types of motivational and emotional input in order to modulate basic cortical information processing according to the physiological state of the animal (Freund, 2003; Freund and Katona, 2007). Such a function might be particularly important in higher-order associative structures, such as the prefrontal cortex. In fact, Whissell et al. (2015) recently showed that CCK+ INs are considerably less abundant compared to PV+ INs in various primary and secondary sensory cortical regions. However, they observed a higher incidence of CCK+ compared to PV+ INs in the mPFC (Whissell et al., 2015), indicating an increased importance of eCB-sensitive CCK+ INs in regulating cortical circuit activity with relevance to higher-order processes.

\subsubsection{Role of endocannabinoid signaling in stress regulation}

The eCB system is widely distributed throughout almost all regions of the brain (Herkenham et al., 1990; 1991; Glass et al., 1997). Indeed, CB1 receptors are considered one of the most abundant G-protein coupled receptors in the central nervous system and have been implicated in the modulation of a wide range of emotional, motivational, and cognitive processes (Kano et al., 2009; McLaughlin et al., 2014; Lutz et al., 2015).

Retrograde eCB-CB1 receptor signaling has been especially linked to mood and stress regulation (McLaughlin et al., 2014; Lutz et al., 2015). Indeed, cannabinoid drugs, such as Cannabis, belong to the most popular recreational drugs worldwide and are consumed primarily because of their relaxing and euphorigenic properties (Williams and Parker, 2001; Terry-McElrath et al., 2009). However, cannabinoid drugs seem to exert dose-dependent biphasic effects on stress and anxiety, with low doses producing anxiolytic and high doses producing anxiogenic behavioral responses, respectively (Patel and Hillard, 2006; Rubino et al., 2007). These biphasic effects are mediated by central activation of CB1 receptors and can be mimicked by local cannabinoid microinfusions into the mPFC, revealing this structure as a central site of action (Rubino et al., 2007; 2008). Indeed, the prefrontal cortex is particularly 
enriched in CB1 receptors (Herkenham et al., 1991; Glass et al., 1997; Eggan and Lewis, 2007) and has an important function in mediating feedback inhibition over stress signaling pathways under normal physiological conditions (McLaughlin et al., 2014; Herman et al., 2016). Upon acute stress, activation of the hypothalamus-pituitary-adrenal (HPA) axis leads to the release of stress hormones, such as glucocorticoids, into the blood stream. These glucocorticoids are in turn transported to different cortical and subcortical stress-regulatory centers, including the prefrontal cortex (Herman et al., 2016). Glucocorticoids activate stresssensitive prefrontal microcircuits, which serve to down-regulate HPA axis activity and terminate stress signaling through different direct and indirect projection pathways, thereby leading to stress recovery following cessation of stressors (Duncan et al., 1993; McKlveen et al., 2013; for review see: Heidbreder and Groenewegen, 2003; McLaughlin et al., 2014; Herman et al., 2016). It has been suggested that retrograde eCB signaling at GABAergic synapses may act as an important gatekeeper of prefrontal feedback inhibition (McLaughlin et al., 2014). Hill et al. (2011b) found that acute stress leads to glucocorticoid-mediated stimulation of prefrontal 2AG signaling, thereby leading to a depression of local synaptic inhibition and possibly gating of stress-regulatory prefrontal circuit activity. Consistently, systemic pharmacological or genetic blockade of CB1 receptors as well as local prefrontal CB1 receptor blockade all lead to enhanced secretion of corticosterone (CORT), the major glucocorticoid stress hormone in rodents (Patel et al., 2004; Hill et al., 2010; 2011b).

The stress-regulatory functions of the prefrontal cortex collapse under conditions of chronic stress. For instance, a considerable body of evidence suggests that chronic exposure to stress or glucocorticoids leads to severe prefrontal circuit dysfunction, homeostatic dysregulation of HPA axis activity, and the development of anxiety- and depression-like phenotypes (Wellman, 2001; Mizoguchi et al., 2003; Cook and Wellman, 2004; Radley et al., 2006; Cerqueira et al., 2007; Yuen et al., 2012; Chiba et al., 2012; Moda-Sava et al., 2019; for review see: Arnsten, 2009; Popoli et al., 2011). These changes have also been linked to a dysfunction in prefrontal eCB signaling (McLaughlin et al., 2014). Indeed, there are several reports of eCB system alterations in the mPFC in animal models of chronic stress, including reduced AEA signaling, altered 2-AG signaling, and upregulation of CB1 receptor binding affinity (Rademacher et al., 2008; Hill et al., 2008; Hill et al., 2010; McLaughlin et al., 2013; Gray et al., 2016). Moreover, some stress-induced cellular and behavioral phenotypes can be mimicked in CB1 receptor knockout mice, such as prefrontal dendritic atrophy and increased anxiety-like behavior (Hill et al., 2011a). Similar findings have been obtained in humans. For example, post-mortem and functional imaging studies revealed changes in the prefrontal eCB system in different psychiatric diseases characterized by stress-induced mood and cognitive dysfunction, including schizophrenia, major depression, posttraumatic stress disorder, and suicide victims (Hungund et al., 2004; Eggan et al., 2008; 2010; Choi et al., 2012; Neumeister et al., 2013). 
These findings suggest an important role of prefrontal eCB-CB1 receptor signaling in stressrelated psychiatric disorders.

\subsection{Scope of the thesis}

The prefrontal cortex has an important role in integrating various cortical and subcortical inputs and mediating top-down control over downstream targets to guide different forms of goaldirected behavior (Miller, 2000; Brown and Bowman, 2002). The prefrontal cortex is highly stress-vulnerable (Arnsten, 2009). This stress vulnerability has been linked to aberrant eCB signaling through presynaptic $\mathrm{CB} 1$ receptors and is thought to contribute to the development of stress-related psychiatric diseases (McLaughlin et al., 2014).

CB1 receptors are abundantly expressed in a subpopulation of locally-acting GABAergic INs, so-called CB1+ INs, thus possibly representing an important cellular substrate for stressinduced changes in eCB signaling (Hill et al., 2011b; McLaughlin et al., 2014). However, their function is only poorly understood, partly due to the lack of reporter lines for target-selective analysis. Moreover, previous studies mainly focused on the function and properties of fastspiking, eCB-insensitive $\mathrm{PV}+\mathrm{INs}$. Indeed, $\mathrm{PV}+\mathrm{INs}$ are frequently considered the most abundant IN type in neocortex (Rudy et al., 2011; Fino et al., 2013; Tremblay et al., 2016). However, the idea of PV+ INs as a dominant source of inhibition is largely based on findings obtained at the primary sensory systems level and it is conceivable that other inhibitory network motifs operate in higher-order neocortical structures. In support of this view, there is evidence suggesting that circuit recruitment of $\mathrm{PV}+$ and $\mathrm{CB} 1+\mathrm{INs}$ is strongly shaped by cortical hierarchy. Whissell et al. (2015) showed that PV+ INs represent the most abundant IN type in primary and secondary sensory cortical regions. However, a different scenario emerges at the level of the mPFC, which was found to be characterized by a higher abundance CCK+ INs, a putative subtype of CB1+ INs (Whissell et al., 2015).

Together, these findings raise the possibility that cortical hierarchy has an important role in shaping the formation of cell type-specific inhibitory circuit motifs of PV+ and $C B 1+$ INs with relevance to stress-induced disease states. Importantly, we recently generated a reporter mouse for CB1+ INs, allowing a direct targeting of these neurons (Winters et al., 2012). We crossed these mice with PV-Cre reporter mice to generate double reporter mice for CB1+ and $\mathrm{PV}+\mathrm{INs}$. In the present study, I sought to determine the distribution and functional circuit properties of these two IN types in mouse mPFC, using a combination of fluorescent imaging and patch-clamp electrophysiology. To assess the role of cortical hierarchy, I compared the inhibitory connectivity of the two IN types in the mPFC with that in primary somatosensory cortex S1 as a representative cortical region for basal sensory processing. Finally, I assessed the developmental trajectories and glucocorticoid-mediated stress vulnerability of prefrontal $\mathrm{CB} 1+$ and $\mathrm{PV}+\mathrm{INs}$ during adolescence as a critical developmental period of heightened prefrontal stress susceptibility. 
2. Materials and Methods 


\subsection{Animal housing conditions}

Mice were housed in groups of 2-5 in standard Blue Line 1285L Type II L cages $(32.5 \times 17.0$ x $14.0 \mathrm{~cm}$; Tecniplast) under controlled laboratory conditions $\left(23^{\circ} \mathrm{C}\right.$ temperature, $60-70 \%$ humidity, and 12/12 h day/night cycle, with lights on at 05:00). Cages were equipped with bedding material. Tissue papers and wood wool served as nesting material. Food and water were provided ad libitum. All mice were weaned and separated into same-sex littermate groups at three weeks of age. Both males and females were used for experiments. The experimental procedures were in accordance with the current European guidelines (Directive 2010/63/EU) and approved by the ethics committee of the local government (Laves, Niedersächsisches Landesamt für Verbraucherschutz und Lebensmittelsicherheit).

\subsection{Mouse lines}

Transgenic PIYCBR double reporter mice (B6;129P2-Pvalbtm1(cre)Arbr/J x B6.CgGt(ROSA)26Sortm3(CAG-EYFP)Hze/J x CB1-tdTomato) were bred in the animal facilities of the European Neuroscience Institute and the Max-Planck-Institute for Experimental Medicine, Göttingen. They were triple-transgenic mice generated on a mixed Agouti x C57BI6/J background, in which PV+ and CB1+ INs were tagged with yellow fluorescent protein (YFP) and tdTomato, respectively. PV+ INs were labeled through cell type-specific expression of Cre recombinase under the Pvalb promoter (PV-Cre), which triggered the loxP site-restricted expression of an inducible YFP (iYFP) in the Rosa26 locus (Srinivas et al., 2001; Hippenmeyer et al., 2005; Madisen et al., 2010). CB1+ INs were labeled through a direct insertion of tdTomato as a second cistron into the Cnr1 gene (CB1-tdTomato) (Winters et al., 2012). Only mice heterozygous for all transgenes were used for experiments. To this aim, male homozygous PIYCBR double reporter mice were always crossed with female C57BI6/J mice. C57BI6/J mice were purchased from Charles River.

\subsection{Genotyping}

Genotyping of PIYCBR double reporter mice was performed using polymerase chain reaction (PCR), followed by agarose gel electrophoresis. First, genomic DNA was isolated from small, 1-2 mm tail samples or ear punches collected after birth or weaning. To this aim, tissue samples were lysed in PBND buffer (10 mM Tris, $50 \mathrm{mM} \mathrm{KCl}, 2.5 \mathrm{~m} \mathrm{M} \mathrm{MgCl}_{2}{ }^{*} 6 \mathrm{H}_{2} \mathrm{O}, 0.1 \mathrm{mg} / \mathrm{ml}$ gelatine, $0.45 \%(\mathrm{v} / \mathrm{v})$ Nonident P40 (NP40) and 0.45\% (v/v) Tween20; $\mathrm{pH}$ 8.3) supplemented with $1: 100$ Proteinase $\mathrm{K}$ solution $\left(20 \mathrm{mg} / \mathrm{ml}\right.$, Ambion) at $55^{\circ} \mathrm{C}$ for at least $3 \mathrm{~h}$ and constant shaking of 800-1000 revolutions per minute (rpm). Subsequently, lysed samples were incubated at $99^{\circ} \mathrm{C}$ for $10 \mathrm{~min}$ to inactivate Proteinase $\mathrm{K}$ and centrifuged at maximum speed (14680 rpm) for $2 \mathrm{~min}$. Supernatants were collected and stored at $4^{\circ} \mathrm{C}$ and served as DNA templates for the following PCR reactions. 
PCR reactions were carried out in FastGene®) Optima HotStart ReadyMix (NIPPON Genetics Europe), a ready-to-use 2x PCR reaction buffer containing a DNA polymerase blend $(0.2 \mathrm{U}$ per $\mu$ reaction), FastGene® Optima buffer, dNTPs $(0.4 \mathrm{mM}$ of each at $1 \mathrm{x}), \mathrm{MgCl}_{2}(4 \mathrm{mM}$ at $1 \mathrm{x}$ ), stabilizers, and an inert loading dye. The ready mix was diluted in $\mathrm{dd}_{2} \mathrm{O}$ and the primers and the DNA template were added. Specific PCR conditions, primer sequences and band sizes of the resulting PCR products are listed in Table 1 and 2.

PCR products were analyzed using agarose gel electrophoresis. Gels were prepared with $1 \%$ agarose in sodium tetraborate buffer ( $5 \mathrm{mM}$ sodium tetraborate decahydrate, Sigma) supplemented with Midori Green Xtra ( $3 \mu \mathrm{l} / 100 \mathrm{ml}$ buffer solution; NIPPON Genetics Europe) to stain DNA fragments. PCR products were run at a voltage of $140-160 \mathrm{mV}$ for $\sim 40 \mathrm{~min}$. Band size was determined by adding a 100 base pair (bp) DNA ladder to the gel (GeneRuler $100 \mathrm{bp}$ PlusDNA Ladder, Thermo Scientific). DNA bands were visualized and imaged with the FAS-V gel documentation system (NIPPON Genetics).

Table 1: $\quad$ PCR conditions for genotyping of PV-Cre, iYFP, and CB1-tdTomato

\begin{tabular}{|c|c|c|c|c|c|}
\hline PCR mixture & & PCR program & & & \\
\hline $\begin{array}{l}\text { 2x FastGene® Optima } \\
\text { HotStart ReadyMix }\end{array}$ & $12.5 \mu \mathrm{l}$ & Cycle step & Temp. & Time & Cycles \\
\hline $50 \mu \mathrm{M}$ fwd WT primer & $0.2 \mu \mathrm{l}$ & & & & \\
\hline $50 \mu \mathrm{M}$ rev WT primer & $0.2 \mu \mathrm{l}$ & Initial denaturation & $95^{\circ} \mathrm{C}$ & $3 \mathrm{~min}$ & \\
\hline $50 \mu \mathrm{M}$ fwd $\mathrm{KI}$ primer & $0.2 \mu \mathrm{l}$ & Denaturation & $95^{\circ} \mathrm{C}$ & $15 \mathrm{~s}$ & \\
\hline $50 \mu \mathrm{M}$ rev $\mathrm{KI}$ primer & $0.2 \mu \mathrm{l}$ & Annealing & $59^{\circ} \mathrm{C}$ & $30 \mathrm{~s}$ & $\times 35$ \\
\hline \multirow[t]{2}{*}{$\mathrm{dd}_{2} \mathrm{O}$} & $11.7 \mu \mathrm{l} /$ & Extension & $72^{\circ} \mathrm{C}$ & $45 \mathrm{~s}$ & \\
\hline & $12.1 \mu \mathrm{l}$ & Final extension & $72^{\circ} \mathrm{C}$ & $2 \min$ & \\
\hline DNA template & $2.0 \mu \mathrm{l}$ & & & & \\
\hline Total volume & $27.0 \mu \mathrm{l}$ & & & & \\
\hline
\end{tabular}

Notes: For genotyping of PV-Cre and iYFP, all four primers for the WT and KI alleles were combined in the same reaction. Genotyping of the CB1-tdTomato WT and KI alleles was performed in separate reactions (with KI primers highlighted in gray), because the amplified DNA sequences shared the same band size. The amount of $\mathrm{ddH}_{2} \mathrm{O}$ for the $\mathrm{CB} 1$-tdTomato WT and $\mathrm{KI}$ reactions was therefore increased to $12.1 \mu \mathrm{l}$ (highlighted in gray) to reach a $1 \mathrm{x}$ concentration of the FastGene® Optima HotStart ReadyMix. Abbreviations: $\mathrm{fwd}=$ forward; $r \mathrm{ev}=$ reverse; $\mathrm{WT}=$ wildtype; $\mathrm{KI}=$ knock-in . 
Table 2: $\quad$ Primer sequences and band sizes for PV-Cre, iYFP, and CB1-tdTomato

\begin{tabular}{|c|c|c|c|}
\hline Gene & Allele & Primer sequence $\left(5^{\prime}-3^{\prime}\right)$ & Band size (bp) \\
\hline \multirow[t]{4}{*}{ PV-Cre } & 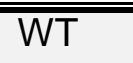 & fwd: CAGAGCAGGCATGGTGACTA & 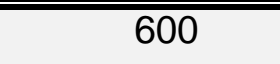 \\
\hline & & rev: AGTACCAAGCAGGCAGGAGA & \\
\hline & $\mathrm{KI}$ & fwd: GCGGTCTGGCAGTAAAAACTATC & 170 \\
\hline & & rev: GTGAAACAGCATTGCTGTCACTT & \\
\hline \multirow[t]{4}{*}{ iYFP } & WT & fwd: AAGGGAGCTGCAGTGGAGTA & 297 \\
\hline & & rev: CCGAAAATCTGTGGGAAGTC & \\
\hline & $\mathrm{KI}$ & fwd: ACATGGTCCTGCTGGAGTTC & 212 \\
\hline & & rev: GGCATTAAAGCAGCGTATCC & \\
\hline \multirow[t]{4}{*}{ CB1-tdTomato } & WT & fwd: GACCTGAGACATGCTTTCC & 300 \\
\hline & & rev: ACCGATGAGACAACAGACTTC & \\
\hline & $\mathrm{KI}$ & fwd: GACCTGAGACATGCTTTCC & 300 \\
\hline & & rev: CGCAATACCGGAGTACTAGC & \\
\hline
\end{tabular}

Abbreviations: $\mathrm{WT}$ = wildtype; $\mathrm{KI}$ = knock-in; fwd = forward; rev = reverse.

\subsection{Electrophysiology}

\subsubsection{Acute slice preparation}

Mice were deeply anesthetized with isoflurane (100\% Forene, Abbvie) and decapitated. The brains were quickly removed and immediately submerged in ice-cold N-methyl-D-glucamine (NMDG) cutting buffer (135 mM NMDG, $1 \mathrm{mM} \mathrm{KCl,} 1.2 \mathrm{~m} \mathrm{M} \mathrm{KH}_{2} \mathrm{PO}_{4}, 1.5 \mathrm{mM} \mathrm{MgSO}_{4}$ * $7 \mathrm{H}_{2} \mathrm{O}$, $0.5 \mathrm{mM} \mathrm{CaCl}_{2}$ * $2 \mathrm{H}_{2} \mathrm{O}, 10 \mathrm{mM}$ D-glucose, and $20 \mathrm{mM}$ choline bicarbonate) saturated with carbogen $\left(95 \% \mathrm{O}_{2}\right.$ and $\left.5 \% \mathrm{CO}_{2}\right)$. Coronal $300 \mu \mathrm{m}$ brain sections were cut in NMDG buffer using a vibrating microtome (Leica VT-1200S) and then transferred to artificial cerebrospinal fluid (ACSF; $119 \mathrm{mM} \mathrm{NaCl}, 2.5 \mathrm{mM} \mathrm{KCl}, 1 \mathrm{mM} \mathrm{NaH}_{2} \mathrm{PO}_{4}, 26 \mathrm{mM} \mathrm{NaHCO}_{3}, 11 \mathrm{mM}$ D-glucose, $1.3 \mathrm{mM} \mathrm{MgSO}_{4}{ }^{*} 7 \mathrm{H}_{2} \mathrm{O}, 2.5 \mathrm{mM} \mathrm{CaCl}_{2}{ }^{*} 2 \mathrm{H}_{2} \mathrm{O}$; carbogenated) for recovery at $35^{\circ} \mathrm{C}$ for $15 \mathrm{~min}$. Thereafter, slices were stored in carbogenated ACSF at room temperature for at least another 45 min before start of electrophysiological recordings.

\subsubsection{Whole-cell patch-clamp recordings}

Standard whole-cell patch-clamp recordings were performed between postnatal days $(P) 50$ 80 unless otherwise indicated. Recordings were carried out at $31 \pm 1^{\circ} \mathrm{C}$ in a recording chamber continuously perfused with carbogenated ACSF at a $2 \mathrm{ml} / \mathrm{min}$ flow rate. Patch pipettes (3-5 $\mathrm{M} \Omega$ ) were pulled from borosilicate glass (KG-33, outer/inner diameter: 1.50/1.00 mm, King Precision Glass Inc) using a micropipette puller (P-1000, Sutter Instruments) and filled with different internal solutions depending on the recording.

Current-clamp (CC) recordings to trigger APs and assess the intrinsic cell excitability were made with K-gluconate-based internal solution (130 mM K-gluconate, $10 \mathrm{mM} \mathrm{KCl}, 10 \mathrm{mM}$ 
phosphocreatine- $\mathrm{Na}_{2}, 10 \mathrm{mM}$ HEPES, $0.2 \mathrm{mM}$ EGTA, $4 \mathrm{mM}$ Mg-ATP, $0.5 \mathrm{mM}$ Na-GTP; pH adjusted to 7.2-7.3 with $\mathrm{KOH}$; $285-290 \mathrm{mOsm}$ ). For voltage-clamp (VC) recordings of GABAergic inhibitory postsynaptic currents (IPSCs), pipettes were filled with $\mathrm{Cl}^{-}$-based internal solution (120 mM CsCl, 15 mM CsMeS, 4 mM TEA-Cl, 5 mM QX314-Br, 20 mM HEPES, 0.4 mM EGTA, 3 mM Mg-ATP, 0.25 mM Na-GTP; pH adjusted to 7.2-7.3 with $\mathrm{CsOH}$, 280$290 \mathrm{mOsm})$. VC recordings of glutamatergic excitatory postsynaptic currents (EPSCs) were made with Cs-gluconate-based internal solution (130 mM Cs-gluconate, $3 \mathrm{mM}$ TEA-Cl, $4 \mathrm{mM}$ QX314-Cl, 20 mM HEPES, 0.4 mM EGTA, 4 mM Mg-ATP, 0.3 mM Na-GTP, pH adjusted to 7.2-7.3 with $\mathrm{CsOH}, 285-295$ mOsm).

Patch-clamp recordings were made from $\mathrm{PV}+\mathrm{INs}, \mathrm{CB} 1+\mathrm{INs}$, and pyramidal neurons (PNs) in $\mathrm{S} 1$ and $\mathrm{mPFC}$, with the latter including both PrL and IL subdivisions. PV+ and CB1+ INs were identified in PIYCBR reporter mice based on their fluorescence using a fixed-stage, infrareddifferential interference contrast microscope (Axio Examiner D1, Zeiss). PNs were identified based on their morphology, lack of fluorescence, and electrophysiological signatures. Input and series resistance were continuously monitored by applying short, hyperpolarizing voltage or current steps. For VC recordings, cells with a series resistance of $>30 \mathrm{M} \Omega$ or a change in series resistance of $>20 \%$ were discarded from quantitative analysis. The liquid junction potential was not corrected. Voltage-drops in CC recordings were compensated via bridge balance. The inter-sweep stimulation frequency was always set at $0.2 \mathrm{~Hz}$. Signals were recorded with a differential ELC-03XS amplifier (npi electronic), filtered at $3 \mathrm{kHz}$, and digitized at $20 \mathrm{kHz}$ using an ITC-18 A/D converter (HEKA). Data were collected with custom-written routines in Igor Pro 7.2 (WaveMetrics) and analyzed with AxoGraph 1.7.4 (John Clemens) and Minianalysis (Synaptosoft).

\subsubsection{Drugs}

Glutamatergic transmission was blocked with the AMPA receptor antagonist NBQX $(2,3-$ dihydroxy-6-nitro-7-sulfamoyl-benzo[f]quinoxaline; $10 \mu \mathrm{M}$ ) and the NMDA receptor antagonist AP5 ((2R)-amino-5-phosphonovaleric acid; $50 \mu \mathrm{M})$. GABAergic transmission was blocked with the $\mathrm{GABA}_{\mathrm{A}}$ receptor antagonist picrotoxin (PTX; $50 \mu \mathrm{M}$ ). eCB signaling was blocked with the neutral CB1 receptor-selective antagonist NESS0327 (8-chloro-1-(2,4-dichlorophenyl)-1,4,5,6tetrahydro-N-1-piperidinyl-benzo[6,7]cyclohepta[1,2-c]pyrazole-3 carboxamide; $1 \mu \mathrm{M}$ ). For miniature EPSC recordings, AP-dependent synaptic neurotransmitter release was blocked using the voltage-gated sodium channel blocker tetrodotoxin (TTX; $1 \mu \mathrm{M}$ ). NBQX, AP5, PTX, and TTX were dissolved in $\mathrm{ddH}_{2} \mathrm{O}$, while NESS0327 was dissolved in dimethyl sulfoxide (DMSO). The different drugs were purchased from Hello Bio, Abcam/Ascent Scientific, Tocris, and Cayman Chemical. 


\subsubsection{Electrophysiological assays}

\subsubsection{Intrinsic Excitability}

Intrinsic excitability recordings were performed in CC mode, with resting membrane potential (RMP) normalized to $-70 \mathrm{mV}$. Intrinsic excitability was assessed by injecting $500 \mathrm{~ms}$ long current pulses into the cell from -200 pA to 600 pA in 50 pA steps. AMPA and NMDA receptormediated glutamatergic transmission was blocked with NBQX and AP5. GABA $A_{A}$ receptormediated inhibitory synaptic transmission was blocked with PTX. The number of APs at each current step was assessed and the first current step eliciting at least one AP was operationally defined as the rheobase current. Current-voltage $(I-V)$ curves were obtained by measuring the change in membrane potential at steady-state induced by each current step until reaching the rheobase current.

\subsubsection{Pairwise recordings}

To study the local inhibitory connectivity, pairwise patch-clamp recordings were made between a presynaptic IN and an adjacent postsynaptic PN, according to a protocol previously used in the NAc (Winters et al., 2012). The presynaptic IN was stimulated in CC mode to elicit APs, while the postsynaptic PN was held at $-70 \mathrm{mV}$ in $\mathrm{VC}$ mode to assess whether IN activation triggered unitary IPSCs (UIPSCs) in the PN. I used a paired-pulse ratio (PPR) assay, in which two consecutive APs were elicited in the presynaptic IN with a 50 ms delay in order to evoke two paired uIPSCs in postsynaptic PNs. The PPR is thought to be inversely correlated to the presynaptic release probability (Pr) (Dobrunz and Stevens, 1997; Zucker and Regehr, 2002) and was measured by dividing the amplitude of the second uIPSC by that of the first. At high Pr synapses, the second synaptic response is typically smaller than the first, leading to pairedpulse depression. At low Pr synapses, the second response is usually larger than the first, leading to paired-pulse facilitation. Presynaptic APs were induced through 2-3 ms long, 9001000 pA current pulses. GABAergic responses were isolated in the presence of NBQX. PNs were randomly sampled within a $100 \mu \mathrm{m}$ radius around the IN soma. Several synaptic parameters were assessed. The connection probability of the two IN types onto PNs was calculated by dividing the number of connected pairs by the number of total pairwise recordings. At connected pairs, the following uIPSC parameters were additionally determined, using averaged responses of 15-30 sweeps: (1) the uIPSC amplitude (including failures), (2) the uIPSC potency (excluding failures), (3) the failure rate (percentage of sweeps, in which the first presynaptic AP failed to trigger uIPSCs in postsynaptic PNs), (4) the PPR, (5), the synaptic delay (i.e., latency between the AP peak and uIPSC onset), (5) the rise time, (6) the half-width, and (7) the decay time constant tau. The rise time was measured at the $10-90 \%$ fragment of the uIPSC rising phase. The decay time constant tau was derived by fitting a single exponential decay function to the decaying element of the UIPSC. Synaptic properties were determined using the average uIPSC induced by the first AP. 


\subsubsection{Depolarization-induced suppression of synaptic inhibition}

$\mathrm{DSI}$ is a form of eCB-dependent short-term synaptic plasticity that was used in IPSC recordings to study the sensitivity of GABAergic synapses to retrograde $\mathrm{eCB}$ signaling. DSI was triggered at connected pairs as described previously (Winters et al., 2012). After a $1 \mathrm{~min}$ baseline period, postsynaptic PNs were depolarized from $-70 \mathrm{mV}$ to $0 \mathrm{mV}$ for $5 \mathrm{~s}$ to trigger eCB release, followed by another 2 min recording. Such depolarization-induced retrograde eCB signaling will transiently suppress GABA release by activating presynaptic CB1 receptors, resulting in a lower $\operatorname{Pr}$ and reduced uIPSC amplitudes. Therefore, the DSI assay was combined with PPR recordings to assess concomitant changes in Pr. Only reliable pairs with failure rates $<0.5$ during baseline were used for analysis. Whenever possible, two such DSI runs were averaged to yield a robust estimate for the strength of DSI. The relative uIPSC amplitude and relative PPR were calculated by normalizing averaged responses from two sweeps immediately after the depolarizing pulse to the $1 \mathrm{~min}$ baseline period. However, sometimes, only failures occurred after postsynaptic PN depolarization and therefore, no PPR could be computed. Always two successive sweeps were averaged.

\subsubsection{Miniature EPSCs}

Miniature EPSCs (mEPSCs) were recorded at $-70 \mathrm{mV}$ in the presence of the voltage-gated sodium channel blocker TTX to assess spontaneous AMPA receptor-mediated synaptic transmission. Under these conditions, glutamatergic events detected in the postsynaptic neuron are caused by spontaneous vesicle fusions with the presynaptic membrane (Wall and Usowicz, 1998; Hsia et al., 1998). The mEPSC amplitude is therefore determined by the quantal size and reflects the postsynaptic response driven by single vesicle fusion events. It can be used therefore as a readout for the strength of AMPA receptor-mediated transmission at single excitatory synapses. The mEPSC frequency represents the number of activated synapses in a given time interval and constitutes a composite measure determined by the vesicle fusion propensity and the number of AMPA receptor-containing synapses. 400 events were analyzed for each neuron. Cumulative probability distributions of mEPSC inter-event intervals and amplitudes were calculated for each individual neuron. To this end, mEPSC interevent intervals and amplitudes were ranked in ascending order and binned into a total of 20 fractions (corresponding to $5 \%$ cumulative fractions of all events per neuron), with each fraction thus containing 20 data points. These raw values were averaged in order to obtain the mean mEPSC inter-event interval and amplitude per fraction. Additionally, the mean mEPSC frequency (the inverse of the inter-event interval) and the mean mEPSC amplitude were calculated from all 400 events per neuron. 


\subsection{In vivo manipulations}

\subsubsection{Chronic corticosterone treatment}

The effects of glucocorticoid signaling on inhibitory synaptic function were tested using exposure to the stress hormone CORT. Mice were chronically treated with CORT through the drinking water using a modified protocol established by Karatsoreos et al. (2010). CORT (HelloBio) was administered at a dose of $0.1 \mathrm{mg} / \mathrm{ml}$ drinking water. To this end, the drug was dissolved in $100 \%$ ethanol $(\mathrm{EtOH})$ at $10 \mathrm{mg} / \mathrm{ml}$ and then further diluted with regular tap water to a final $1 \%$ concentration (Karatsoreos et al., 2010). After delivery, mice were first habituated to the new housing conditions for $\sim 5$ days and then treated with CORT from P30 for a period of at least 20 days before start of behavioral and electrophysiological experiments. Drug treatment was continued throughout the experiments. Vehicle controls received drinking water containing $1 \% \mathrm{EtOH}$ during the same time frame. Water bottles were regularly exchanged every 5-7 days in order to account for possible changes in drug stability. The body weight of the mice was assessed every 5 days.

\subsubsection{Open field test}

Stress-related behavioral changes in CORT-treated mice were examined after a treatment phase of 20 days at P50 using the open field test, a standard assay for anxiety-related behavior in rodents (Prut and Belzung, 2003). The open field test was conducted in a squared $40 \times 40$ $x 40 \mathrm{~cm}$ chamber with a white floor and gray side walls under dim-light conditions. Mice were carefully positioned in the center of the arena and allowed to freely explore the open field arena during a 10 min session. Behavior was recorded by a USB video camera mounted centrally above the arena and captured with IC capture software 2.4 (The Imaging Source). Videos were analyzed off-line using ANY-maze software (Stoelting). The time spent in the center of the open field was used to assess CORT-induced changes in anxiety-related behavior, with lower scores indicating higher anxiety-like behavior (Simon et al., 1994; Choleris et al., 2001). The center was defined as the inner $30 \times 30 \mathrm{~cm}$ zone of the arena. Total distance traveled served as a measure for general locomotor activity.

\subsection{Histology}

\subsubsection{Transcardial perfusion}

PIYCBR reporter mice were deeply anesthetized with isoflurane and perfused through the left ventricle. The blood was washed with $\sim 40 \mathrm{ml}$ phosphate-buffered saline (PBS; $137 \mathrm{mM} \mathrm{NaCl}$, $2.7 \mathrm{mM} \mathrm{KCl}, 10 \mathrm{mM} \mathrm{Na}_{2} \mathrm{HPO}_{4} ; 1.8 \mathrm{mM} \mathrm{KH}_{2} \mathrm{PO}_{4} ; \mathrm{pH} \sim 7.4$ ), followed by $\sim 40 \mathrm{ml} 4 \%$ paraformaldehyde (PFA) in PBS ( $\mathrm{pH} 6.9$ ). The brains were carefully removed and stored in 4\% PFA overnight for $24 \mathrm{~h}$ post-fixation. Thereafter, brains were transferred to PBS and cut into $100 \mu \mathrm{m}$ slices using a vibrating microtome (Leica VT-1200S). Slices were mounted onto 
glass slides (Labsolute/Th.Geyer) using MOWIOL-DABCO mounting medium (0.1 M Tris, $2.5 \%$ Glycerol, $10 \% \mathrm{MOWIOL}$, and $2.5 \% \mathrm{DABCO}$ ) and stored at $-20^{\circ} \mathrm{C}$ until further use.

\subsubsection{Fluorescence microscopy}

For cell density analysis of $C B 1+$ and $P V+I N s$, epifluorescent images were acquired at $10 x$ using an ECLIPSE Ts2R microscope equipped with NIS-Elements AR imaging software (Nikon). PV+ and CB1+ IN cell bodies were detected automatically with the NIS-Elements AR spot detection function, using the following parameters: method: "Bright, Different Sizes"; output: circular area; diameter: $12 \mu \mathrm{m}$, contrast: 130. Detection parameters were kept constant throughout experimental conditions. Cell densities were calculated for individual mice by averaging values from six different images per cell type and cortical region. High-magnification images were acquired at 40x with a confocal LSM710 microscope (Carl Zeiss) using ZEN black imaging software.

\subsection{Statistical analysis}

Data were analyzed using a combination of different parametric and non-parametric analyses. Whenever possible, data were analyzed with parametric $t$-tests or one-, two-, or three-way between-subject and repeated-measures ( $\mathrm{rm}-$ ) analyses of variance (ANOVAs), followed by post-hoc tests when indicated. Degrees of freedom for the $F$-tests in rm-ANOVAs were adjusted using Huyn-Feldt correction in case sphericity was violated. However, the uIPSC parameters at synaptically connected pairs usually displayed non-normal error distributions and heterogeneity of variance and were therefore always analyzed with non-parametric twoway aligned-rank transform- (art-) ANOVAs (Wobbrock et al., 2011), which were followed by post-hoc Mann-Whitney $U$ tests whenever indicated. The connection probability was analyzed with binary logistic regression and/or Fisher's exact tests. Hierarchical cluster analysis was performed on z-standardized variables using Ward fusion algorithm and the squared Euclidian distance as linkage distance. Normality and homogeneity of variance were tested with ShapiroWilk and Levene tests. Post-hoc tests were always adjusted for alpha-error accumulation using Bonferroni-Holm correction. Data are presented as box plots (with whiskers representing the $10^{\text {th }}$ and $90^{\text {th }}$ percentile) or as mean \pm the standard error of the mean (SEM). Hierarchical clustering results are reported using dendrograms and corresponding heat maps depicting properties of individual cluster members as color-coded z-values. For electrophysiological recordings, $n$ indicates the number of cells or synaptically connected pairs and $m$ the number of mice per experimental group. For the connection probability, the number of synaptically connected pairs relative to all recorded pairs per condition is given in parenthesis. All statistical tests were performed two-tailed, except for one-sample $t$-tests in DSI experiments. Nominal alpha error was set to $\alpha=0.05$. 
3. Results 


\subsection{CB1+ and PV+ interneurons represent distinct cell types in neocortex}

In neocortex, CB1 receptors preferentially localize onto presynaptic terminals of CCK+ INs, while no expression is seen in PV+ INs (e.g. Marsicano and Lutz, 1999), suggesting that CB1+ and PV+ INs represent distinct IN types. However, we previously found that the two IN types substantially overlap in the NAc (Winters et al., 2012), raising the question as to whether they indeed represent separate neuron populations.

I therefore first confirmed that $\mathrm{CB} 1+$ and $\mathrm{PV}+\mathrm{INs}$ represent distinct cell types in neocortex. To this end, I prepared forebrain slices from double reporter mice, in which PV+ INs are tagged with YFP and CB1+ INs with tdTomato. I then performed confocal imaging in the MPFC, an important higher-order neocortical structure for cognitive functioning (Miller, 2000; Figure 1A). The tdTomato-positive CB1+ INs and YFP-positive PV+ INs populated similar regions within the mPFC (Figure 1B). However, there were no cells simultaneously expressing both tdTomato and YFP. Thus, the two reporter signals did not co-localize, indicating that the two IN types represent non-overlapping neuron classes (Figure 1C).

Next, I performed whole-cell patch-clamp recordings in mPFC L2/3 and assessed the intrinsic membrane and firing properties of the two IN types in response to sustained current injections (Figure 1D). As expected, PV+ INs showed fast-spiking behavior with high AP firing rates and virtually no frequency adaptation (Figure 1E). By contrast, CB1+ INs were either regularspiking or irregular-spiking/fast-accommodating and thus characterized by a strongly adapting firing pattern (Figure 1F-G). This was also reflected in the cell type-specific changes in the AP firing rates in response to current injections of increasing intensity. PV+ INs showed a nearlinear increase in their AP frequency with increasing current step intensity, indicating little firing rate adaptation (Figure $1 \mathrm{H}$ ). In contrast, the AP frequency was substantially lower in $\mathrm{CB} 1+\mathrm{INs}$ and rapidly reached a plateau, indicating strong firing rate adaptation (current intensity: $F_{(2.68 \text {, }}$ $58.90)=60.73, p<0.001$; current intensity $x$ cell type: $F_{(2.68,58.90)}=33.71, p<0.001$; two-way rmANOVA; $n / m=12 / 5$ per cell type; Figure $1 \mathrm{H}$ ). In order to assess the intrinsic excitability, I further obtained $I-V$ curves for the two IN types by measuring the change in membrane potential in response to hyper- and depolarizing current steps. In both IN types, increasing the current step intensity led to near-linear changes in the magnitude of the resulting membrane potential deflection (Figure 1I). However, at hyperpolarizing current steps, CB1+ INs showed stronger changes in membrane potential and thus steeper I-V curves compared to $\mathrm{PV}+\mathrm{INs}$, indicating higher excitability (-200 to $0 \mathrm{pA}$; current intensity: $F_{(1.09,23.87)}=376.46, p<0.001$; current intensity $x$ cell type: $F_{(1.09,23.87)}=11.17, p=0.002$; two-way rm-ANOVA; Figure 1I). Additionally, they required less depolarizing current to fire APs, as evidenced by a lower rheobase current $\left(t_{(18.58)}=3.47, p=0.003\right.$; Figure $\left.1 \mathrm{~J}\right)$, and were generally more depolarized at resting potential compared to $\mathrm{PV}+\mathrm{INs}\left(t_{(22)}=-10.79, p<0.001\right.$; Figure $\left.1 \mathrm{~K}\right)$. 
Together, these data demonstrate that $\mathrm{PV}+$ and $\mathrm{CB} 1+$ INs represent non-overlapping and physiologically distinct IN types in neocortex. Of note, CB1+ INs were either regular- or irregular-spiking, possibly indicating that at least two different subtypes can be distinguished based on their firing properties.
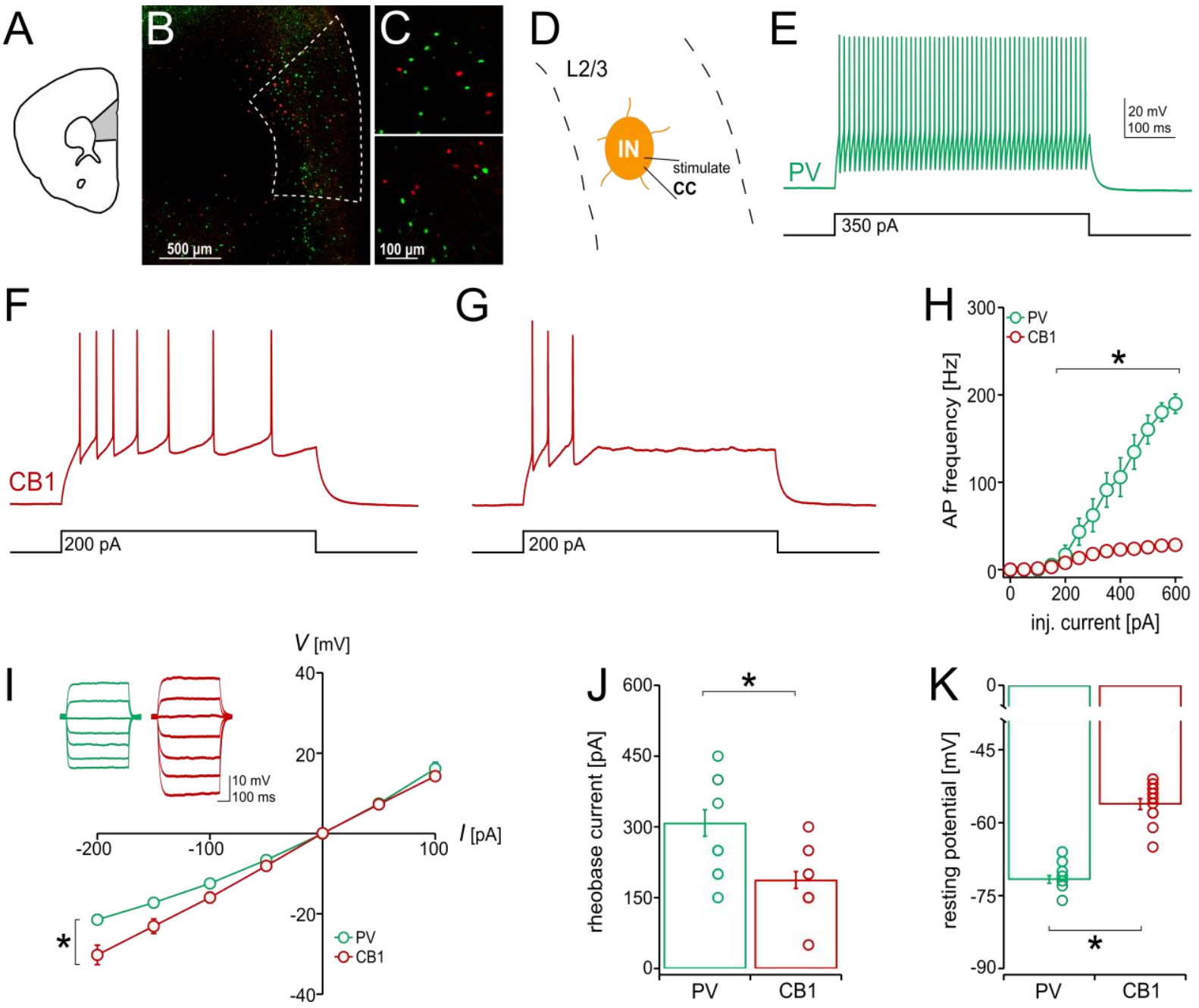

Figure 1: $\mathrm{PV}+$ and $\mathrm{CB} 1+$ interneurons represent functionally distinct cell types in prefrontal cortex. A) Schematic illustration of the mPFC (highlighted in gray). B) Confocal forebrain image from a double reporter mouse, in which $\mathrm{CB} 1+$ and $\mathrm{PV}+\mathrm{INs}$ are tagged with tdTomato (red) and YFP (green), respectively. The position of the mPFC is indicated by the dotted line. C) High-magnification confocal images from different positions within the mPFC. D) Illustration of intrinsic excitability recordings. CB1+ and PV+ INs were recorded in current-clamp (CC) mode and stimulated with hyper- and depolarizing $500 \mathrm{~ms}$ current steps. Resting potential was always normalized to $-70 \mathrm{mV}$. Recordings were performed in mPFC L2/3 in the presence of NBQX $(10 \mu \mathrm{M})$, AP $-5(50 \mu \mathrm{M})$, and PTX $(50 \mu \mathrm{M})$ to block glutamatergic and GABAergic transmission. E-G) Example traces showing the firing patterns of (E) a fast-spiking $P V+I N$, (F) a regular-firing $C B 1+I N$, and (G) an irregular-firing $C B 1+I N$ in response to $500 \mathrm{~ms}$ suprathreshold current steps. H) Summary graph depicting the change in action potential (AP) frequency with increasing current step intensity, using a $50 \mathrm{pA}$ step size. I) $\mathrm{l}-\mathrm{V}$ curves depicting the change in membrane potential $V$ in response to hyper- and depolarizing current steps $I$, using a $50 \mathrm{pA}$ step size. Inset: Example traces depicting the change in membrane potential in a PV+ and CB1+ IN induced by 50 pA current steps from -200 pA to 100 pA. J) Rheobase current, i.e., the minimal current intensity eliciting at least one AP. K) Resting membrane potential. Data are presented as mean \pm SEM. Dots in $\mathbf{J}$ and $\mathbf{K}$ represent individual data points. $n / m=12 / 5$ for both cell types. Data were analyzed with two-way rm-ANOVA or unpaired $t$-tests. * $p<0.05$.

\subsection{The distribution of $\mathrm{CB} 1+$ and $\mathrm{PV}+$ interneurons varies across cortical regions}

$\mathrm{PV}+\mathrm{INs}$ are widely considered the most abundant IN type in neocortex. Indeed, they can make up to $40 \%$ of the whole IN population (Lee et al., 2010a; Whissell et al., 2015). However, such estimates are usually derived from analyses in sensory cortices, e.g. the primary 
somatosensory cortex $\mathrm{S} 1$, while a different scenario might exist in other cortical regions. In fact, Whissell et al. (2015) observed a higher proportion of CCK+ INs in the mPFC and other higher-order structures. Importantly, CCK+ INs strongly express the CB1 receptor (Marsicano and Lutz, 1999), suggesting that CB1+ and PV+ INs are differentially distributed in sensory and higher-order neocortical regions. Using double reporter mice, I therefore assessed the cell densities of CB1+ and PV+ INs in layers L2/3 and L5/6 of S1 and mPFC.
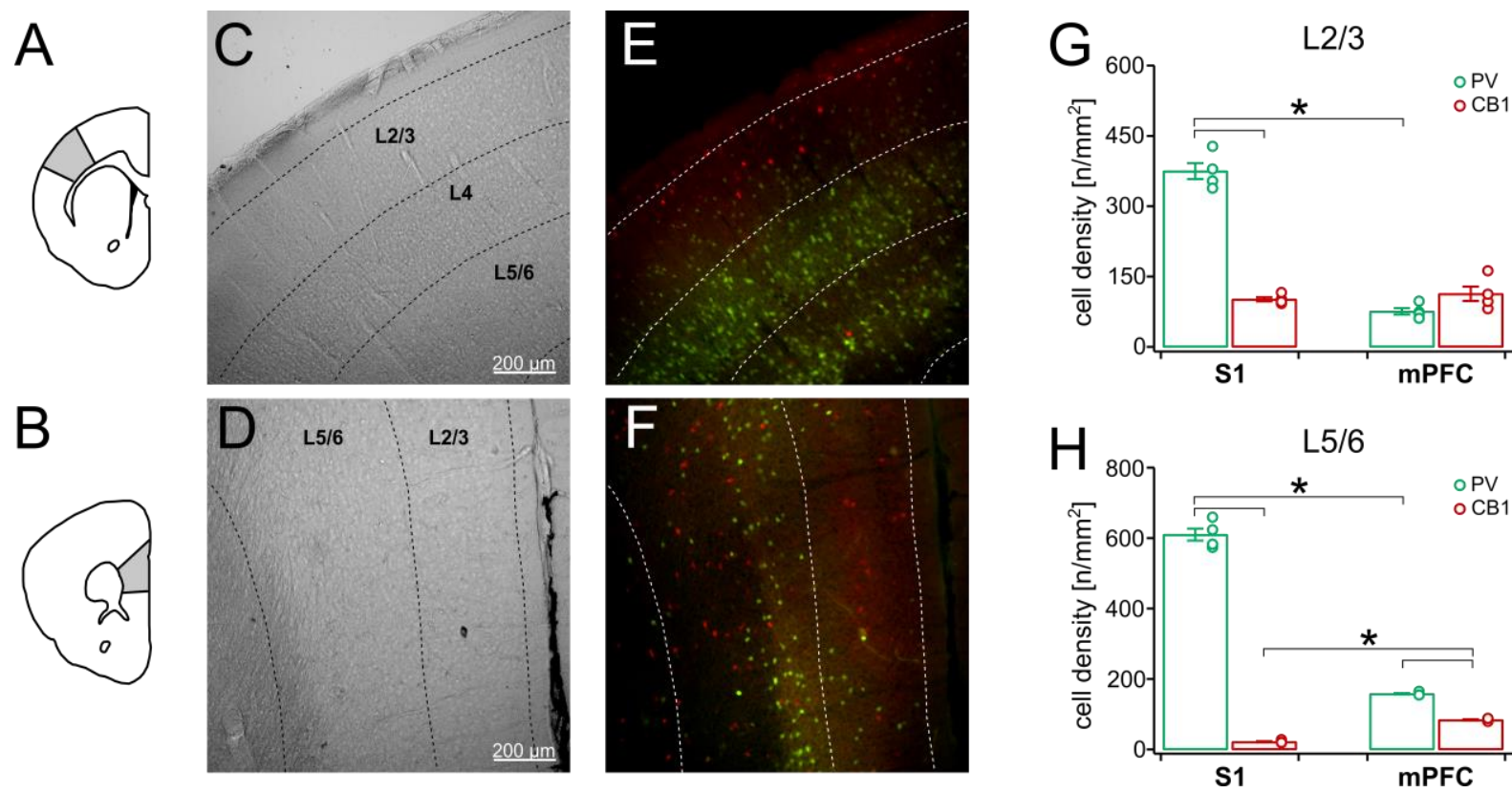

Figure 2: The abundance of $\mathrm{PV}+$ and $\mathrm{CB} 1+$ interneurons converges from somatosensory towards prefrontal cortex. A-B) Schematic illustration of $\mathbf{A}$ ) the $\mathrm{S} 1$ and $\mathbf{B}) \mathrm{mPFC}$ (highlighted in gray). C-D) Example bright-light images of (C) S1 and (D) mPFC obtained from double reporter mice. The different layers are indicated by dotted lines. E-F) Epifluorescence images of C-D) showing the distribution of PV+ (green) and CB1+ INs (red) in the different layers of S1 and mPFC. G-F) Summary graphs comparing the cell density of PV+ and CB1+ INs between $\mathrm{S} 1$ and $\mathrm{mPFC}$ for supragranular layers L2/3 and infragranular layers L5/6. Data are presented as mean \pm SEM. Dots represent individual data points. $n=4$ mice for both cell types. Data were analyzed with two-way rm-ANOVA followed by Bonferroni-Holm adjusted paired and unpaired $t$-tests. ${ }^{*} p<0.05$.

In line with findings by Whissell et al. (2015), I found that PV+ INs heavily populated most layers of $\mathrm{S} 1$. In contrast, $\mathrm{CB} 1+\mathrm{INs}$ were generally sparse. The pattern completely changed in the mPFC, where the number of the two IN types was highly comparable (Figure $2 A-F$ ). This equilibration was most pronounced in layer $L 2 / 3$ (cell type: $F_{(1,6)}=64.24, p<0.001$; region: $F_{(1,6)}=120.09, p<0.001$; cell type $\mathrm{x}$ region: $F_{(1,6)}=140.33, p<0.001$; two-way rm-ANOVA with region as within-subject factor; $m=4$ mice; Figure $2 G$ ). Specifically, while $P V+I N s$ were at least threefold more abundant than CB1+ INs in S1 L2/3 ( $p<0.001)$, the number was more balanced between the two IN types in mPFC L2/3 ( $p=0.297$; post-hoc Bonferroni-Holm adjusted unpaired $t$-tests), with $\mathrm{CB} 1+\mathrm{INs}$ descriptively showing even higher numbers (Figure 2G). The equilibrating effect was mainly driven by a regional decrease in PV+ IN density between $\mathrm{S} 1$ and mPFC ( $p=0.002)$, while CB1+ INs stayed nearly identical across regions ( $p>0.999$; posthoc Bonferroni-Holm adjusted paired $t$-tests; Figure 2G). An almost identical picture emerged when comparing the density of the two IN types in L5/6 between the two regions (cell type: $F_{(1,6)}=1235.43, p<0.001$; region: $F_{(1,6)}=338.45, p<0.001$; cell type $x$ region: $F_{(1,6)}=589.51$, 
p<0.001; two-way rm-ANOVA; Figure 2H). In fact, CB1+ INs were almost absent in S1 layer L5/6 and hence $\sim 30$-fold less abundant than PV+ INs $(p<0.001)$. In the MPFC, the density of CB1+ INs was still twofold lower, but strongly converged with that of PV+ INs ( $p<0.001$; posthoc Bonferroni-Holm adjusted unpaired $t$-tests). This was driven by both a strong decrease in the density of PV+ INs $(p<0.001)$ and a prominent increase in the density of CB1+ INs across regions ( $p<0.001$; post-hoc Bonferroni-Holm-adjusted paired $t$-tests; Figure $2 \mathrm{H}$ ). Together, these data suggest that while CB1+ INs only represent a secondary cell type compared to PV+ INs in sensory cortices, their relative abundance largely converges with that of PV+ INs at the level of higher-order structures, such as the mPFC.

\subsection{The number and properties of GABAergic synapses formed by CB1+ and PV+ interneurons vary across cortical regions}

Besides their high abundance, $\mathrm{PV}+\mathrm{INs}$ are also known to densely innervate neocortical excitatory networks via perisomatic GABAergic synapses and are therefore often considered a dominant source of inhibitory control in neocortex (e.g. Packer and Yuste, 2011; Pfeffer et al., 2013). However, such findings are usually restricted to sensory areas. My results so far indicate that a different circuit motif might exist in the prefrontal cortex, which seems to be populated by another significant IN type besides PV+ INs, namely CB1+ INs. Specifically, while $\mathrm{PV}+\mathrm{INs}$ represented a more abundant cell type compared to $\mathrm{CB} 1+\mathrm{INs}$ in $\mathrm{S} 1$, their occurrence was largely balanced in the mPFC. Using patch-clamp electrophysiology, I sought to determine whether the regional differences in the abundance of the two IN types are also mirrored by corresponding differences in their inhibitory connectivity. I focused on L2/3 since CB1+ INs in S1 only occurred in sufficiently high number in this layer.

\subsection{1. $\quad C B 1+$ and $P V+$ interneurons form GABAergic synapses}

I first confirmed that the two IN types formed GABAergic synapses onto glutamatergic PNs. To this end, I performed pairwise patch-clamp recordings between a presynaptic IN type and an adjacent PN in S1 and mPFC L2/3. The IN was held in CC mode to trigger APs through short depolarizing current injections, while the PN was held in VC mode to record IPSCs in order to test whether the recorded pair was synaptically connected (Figure $3 \mathrm{~A}$ ).

In unconnected pairs, postsynaptic IPSCs occurred randomly and were not associated with presynaptic IN firing. In synaptically connected pairs, IN firing instead systematically triggered so-called uIPSCs in PNs, which were time-locked to the occurrence of presynaptic APs and usually characterized by fast kinetics, irrespective of whether PV+ or CB1+ INs served as presynaptic partners (Figure 3B, C). Importantly, these uIPSCs were completely blocked at both types of synapses and in both cortical regions following bath application of the $\mathrm{GABA}_{\mathrm{A}}$ receptor antagonist PTX $(0.1 \mathrm{mM})$ (time: $F_{(4.68,46.77)}=64.60$, $p<0.001$; time $x$ cell type: $F_{(4.68 \text {, }}$ 46.77) $=0.70, p=0.616$; time $\times$ region: $F_{(4.68,46.77)}=0.91, p=0.475$; time $\times$ cell type $\times$ region: $F_{(4.68 \text {, }}$ 
46.77) $=0.64, p=0.659$; three-way rm-ANOVA; $n=3-4$ cells from $m=2-4$ mice per cell type / region; Figure 3B-E). Together, these results demonstrate that $\mathrm{CB} 1+$ and $\mathrm{PV}+\mathrm{INs}$ form GABAergic synapses onto PNs in neocortex.

A

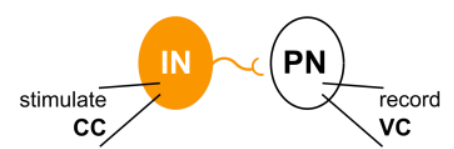

B

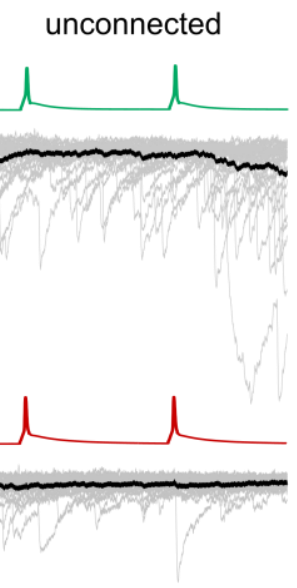

connected

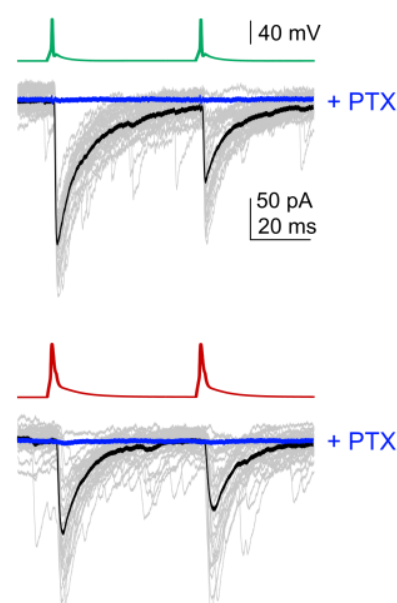

C

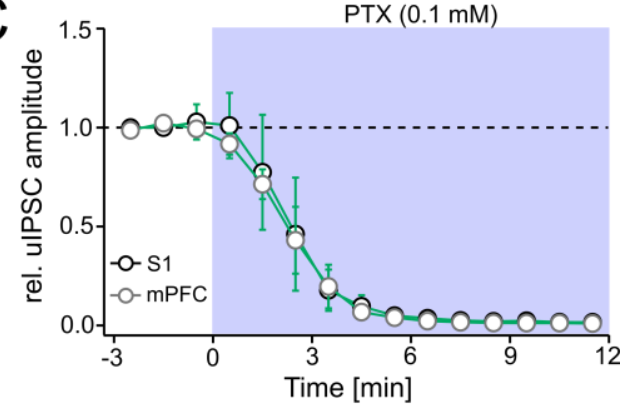

$\mathrm{D}$

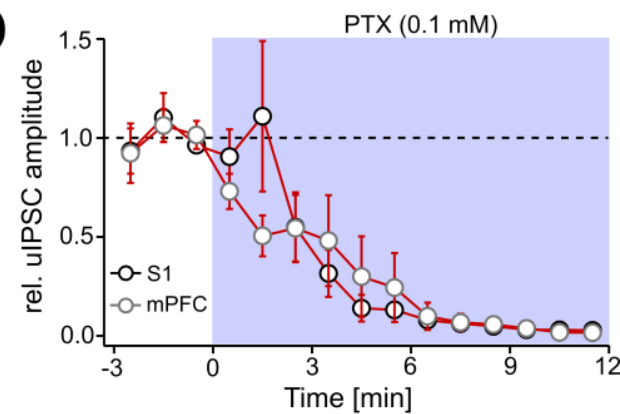

Figure 3: $\mathrm{PV}+$ and $\mathrm{CB} 1+$ interneurons form GABAergic synapses onto neocortical pyramidal neurons. A) Illustration of recording configuration. A presynaptic IN was stimulated in current-clamp (CC) mode to elicit action potentials (APs), while an adjacent PN was held at $-70 \mathrm{mV}$ in voltage-clamp (VC) mode to record IPSCs. Recordings were performed in S1 and mPFC L2/3 in the presence of NBQX $(10 \mu \mathrm{M})$ to block AMPA receptor-mediated glutamatergic transmission. B) Example traces of unconnected and synaptically connected PV-to-PN pairs (upper panel) and CB1-to-PN pairs (lower panel). PV+ (green) and CB1+ INs (red) were stimulated to fire two consecutive APs, while IPSCs were recorded in PNs (black: average of 20-30 sweeps; gray: individual sweeps). At synaptically connected but not unconnected pairs, IN firing triggered unitary IPSCs (uIPSCs) in PNs, which were time-locked to the occurrence of presynaptic APs and fully blocked by PTX bath application $(0.1 \mathrm{mM}$; highlighted in blue). C-D) Summary graphs depicting the blockade of uIPSCs following PTX bath application at (C) synaptically connected PV-to-PN and (D) CB1-to-PN pairs in S1 (black) and mPFC (gray). Shown is the change in the baseline-normalized, relative uIPSC amplitude. Data are presented as mean \pm SEM. PV-to-PN pairs: $n / m=3 / 3$ for $S 1, n / m=4 / 3$ for mPFC; CB1-to-PN pairs: $n / m=4 / 4$ for $S 1, n / m=3 / 2$ for $m P F C$.

\subsubsection{GABAergic synapses made by $\mathrm{CB} 1+$ but not $\mathrm{PV}+$ interneurons are sensitive to retrograde endocannabinoid signaling}

A distinguishing feature of $\mathrm{CB} 1+\mathrm{INs}$ is their sensitivity to retrograde eCB signaling. Therefore, I tested a subset of connected pairs for DSI, an eCB-dependent form of short-term plasticity, in which short postsynaptic depolarization triggers retrograde eCB signaling in $\mathrm{PNs}$ to transiently suppress GABA release from presynaptic terminals that express CB1 receptors (e.g. Wilson et al., 2001; Wilson and Nicoll, 2001; Figure 4A).

At CB1-to-PN pairs, PN depolarization transiently decreased the relative uIPSC amplitude by $25.0 \%\left(t_{(13)}=-2.24, p=0.022\right.$ vs. baseline; one-tailed one-sample $t$-test collapsed across regions; $n / m=14 / 11$; Figure 4B-D), indicating DSI. Consistently, the decrease was short-lived (Figure 4B) and associated with a concomitant increase in the relative PPR $\left(t_{(12)}=1.91, p=0.041\right.$ vs. baseline; $n / m=13 / 10)$, indicating a reduced $\operatorname{Pr}$ and thus a presynaptic mode of action (Figure 4E). Moreover, the decrease in relative uIPSC amplitudes was completely 
A

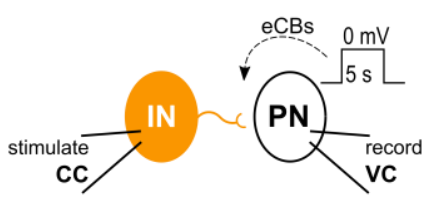

B

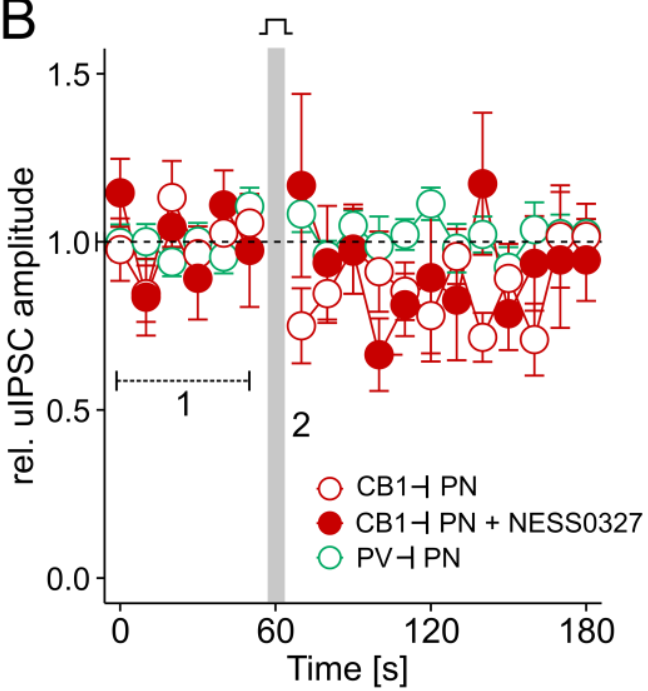

C
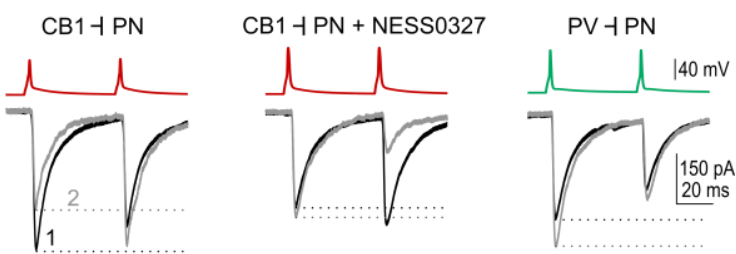

D

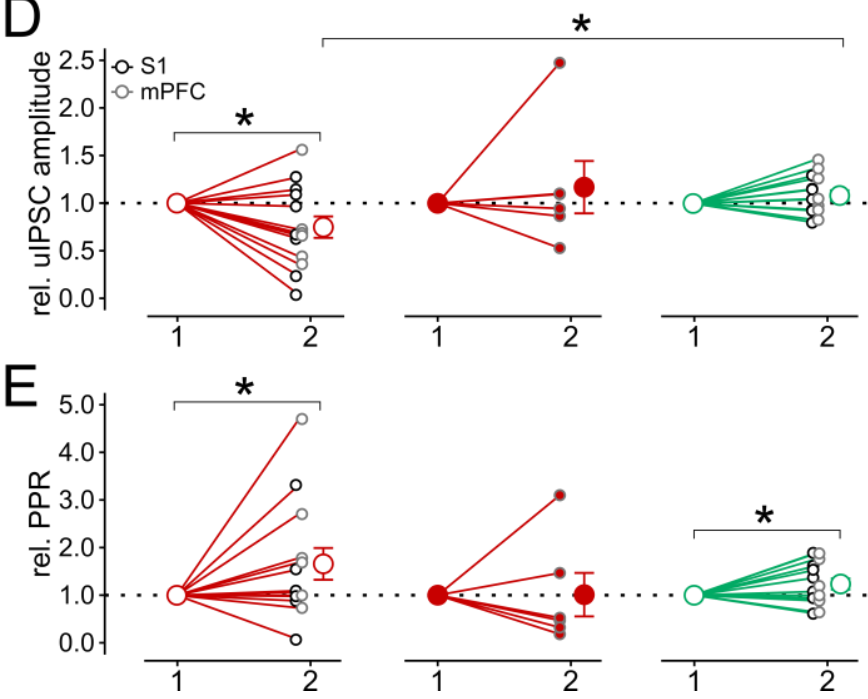

Figure 4: $\mathrm{CB1}+$ but not PV+ interneurons exhibit endocannabinoid-dependent depolarization-induced suppression of synaptic inhibition. A) Illustration of DSI induction protocol. A pairwise recording was made from a synaptically connected IN-to-PN pair. The postsynaptic PN was transiently depolarized from $-70 \mathrm{mV}$ to $0 \mathrm{mV}$ for a duration of $5 \mathrm{~s}$ in order to trigger retrograde eCB signaling. B) Summary graph depicting the time course of DSI in synaptically connected CB1-to-PN pairs (recorded in standard ACSF), CB1-to-PN pairs + NESS0327 (1 $\mu \mathrm{M})$, and PV-to-PN pairs. Postsynaptic PN depolarization was performed after a $60 \mathrm{~s}$ baseline period (indicated by the gray bar). Shown is the baseline-normalized, relative uIPSC amplitude averaged over two consecutive sweeps, collapsed across S1 and mPFC. C) Example traces of average uIPSCs recorded in PNs during the $60 \mathrm{~s}$ baseline period (1, black) and the 10 s period immediately after PN depolarization (2, gray) for a CB1-to-PN pair, CB1-to-PN pair + NESS0327, and a PV-to-PN pair. D-E) Change in (D) the relative uIPSC amplitude and (E) the relative PPR from the $60 \mathrm{~s}$ baseline period (1) to the $10 \mathrm{~s}$ period following PN depolarization (2), shown separately for pairs recorded in S1 (black) and mPFC (gray). Values were obtained by normalizing to the $60 \mathrm{~s}$ baseline period before PN depolarization. Data are presented as mean \pm SEM. Dots in $\mathbf{D}$ and $\mathbf{E}$ represent individual data points. CB1-toPN pairs: $n / m=8 / 6$ for $S 1, n / m=6 / 5$ for mPFC; CB1-to-PN pairs + NESS0327: $n / m=6 / 3$, recordings in mPFC only; PV-to-PN pairs: $\mathrm{n} / \mathrm{m}=7 / 5$ for $\mathrm{S} 1, \mathrm{n} / \mathrm{m}=7 / 4$ for $\mathrm{mPFC}$. Of note, one CB1-to-PN pair in mPFC only showed failures following PN depolarization and therefore had to be excluded from the PPR analysis. Data were analyzed with one-tailed one-sample $t$-tests, unpaired $t$-tests, or two-way ANOVA. * $p<0.05$.

blocked in the presence of the CB1 receptor-selective antagonist NESS0327 $(1 \mu \mathrm{M} ; n / m=6 / 3$, recordings in mPFC only), suggesting that the effect was mediated by retrograde eCB signaling $\left(t_{(5)}=0.62, \quad p>0.999\right.$ vs. baseline; Figure 4C-D). However, the difference did not reach significance compared to standard ACS recordings $\left(t_{(18)}=-1.71, p=0.104\right.$; unpaired, two-tailed $t$-test), possibly due to high variability. NESS0327 also blocked the relative increase in PPR $\left(t_{(5)}=0.02, p=0.491\right.$ vs. baseline), but again no significant difference compared to standard ACSF recordings was seen $\left(t_{(17)}=1.09, p=0.293\right.$; Figure 4E). Interestingly, the basal synaptic strength and release properties at CB1-to-PN pairs remained largely unaffected in the presence of NESS0327 (Supplementary Figure 1). No DSI was induced at PV-to-PN pairs, only showing a minimal increase in PPR but no change in the uIPSC amplitude (relative uIPSC amplitude: $t_{(13)}=1.54, p>0.999$ vs. baseline; relative PPR: $t_{(13)}=2.01, p=0.033$ vs. baseline; $n / m=14 / 9$ collapsed across regions; Figure 4B-E). Importantly, this synapse-specific presence 
of DSI at GABAergic synapses made by $\mathrm{CB} 1+$ but not PV+ INs was consistently seen in both cortical regions (cell type: $F_{(1,24)}=6.68, p=0.016$; region: $F_{(1,24)}=0.06, p=0.804$; cell type x region: $F_{(1,24)}=0.15, p=0.705$; two-way ANOVA for relative uIPSC amplitude; $\mathrm{n} / \mathrm{m}=6-7$ pairs from $4-6$ mice per cell type / region; Figure 4D). Descriptively, similar differences between the two IN types were evident for the relative PPR, but the between-group effects were not significant (as opposed to the within-group comparisons), again probably due to the high variability (cell type: $F_{(1,23)}=1.37, p=0.207$; region: $F_{(1,23)}=0.83, p=0.321$; cell type $x$ region: $F_{(1,23)}=1.44, p=0.196$; twoway ANOVA; $n / m=6-7$ pairs from 4-6 mice per cell type / region; Figure 4E). Together, these results demonstrate a circuit-wide selective sensitivity to retrograde $\mathrm{ECB}$ signaling at neocortical synapses made by CB1+ but not PV+ INs.

\subsubsection{The inhibitory connectivity of $\mathrm{CB1}+$ and $\mathrm{PV}+$ interneurons varies between cortical regions}

Besides the differences in eCB sensitivity, inhibitory synapses made by $\mathrm{CB} 1+$ INs differed from those made by PV+ INs in several other basal properties. In both cortical regions, CB1+ INs formed weaker GABAergic synapses onto PNs compared to PV+ INs, as evidenced by lower uIPSC amplitudes (cell type: $F_{(1,128)}=17.13, p<0.001$; region: $F_{(1,128)}=0.08, p=0.773$; cell type $\mathrm{x}$ region: $F_{(1,128)}=0.03, p=0.853$; two-way art-ANOVA; $\mathrm{n} / \mathrm{m}=30-34$ connected pairs from 14-18 mice per cell type / region; Figure 5A-C). This reduced synaptic strength was also reflected in a lower uIPSC potency (uIPSC amplitude without failures; cell type: $F_{(1,128)}=9.98, p=0.002$; region: $F_{(1,128)}=0.03, p=0.866$; cell type x region: $F_{(1,128)}=0.02, p=0.894$; two-way art-ANOVA; Figure 5D). Moreover, CB1+ INs frequently formed highly unreliable synapses, characterized by a high incidence of transmission failures, while PV+ INs almost exclusively formed reliable synapses without transmission failures (cell type: $F_{(1,128)}=26.45, p<0.001$; region: $F_{(1,128)}=0.94$, $p=0.334$; cell type $x$ region: $F_{(1,128)}=0.55, p=0.459$; two-way art-ANOVA; Figure $\left.5 \mathrm{E}\right)$, indicating a lower Pr at synapses made by CB1+ INs. In large agreement, uIPSCs at CB1-to-PN pairs exhibited a rather high PPR, often reflecting paired-pulse facilitation (PPR $>1.0$ ), while PV-toPN pairs almost only displayed paired-pulse depression (PPR $<1.0$; Figure 5F). However, the difference in PPR was region-specific (cell type: $F_{(1,127)}=2.17, p=0.143$; region: $F_{(1,127)}=2.40$, $p=0.124$; cell type $x$ region: $F_{(1,127)}=11.23, p=0.001$; two-way art-ANOVA) and seen only in mPFC ( $p=0.009)$, but not $S 1(p=0.299)$. This was driven by a trend for a higher PPR in mPFC compared to $\mathrm{S} 1$ for CB1-to-PN pairs $(p=0.086)$ and a trend for a lower PPR for PV-to-PN pairs ( $p=0.071$; post-hoc Bonferroni-Holm adjusted Mann-Whitney $U$ tests; Figure 5F). Moreover, uIPSCs occurred with a longer synaptic delay at CB1-to-PN than PV-to-PN pairs (cell type: $F_{(1,128)}=79.64, p<0.001$; region: $F_{(1,128)}=0.24, p=0.622$; cell type $x$ region: $F_{(1,128)}=0.18, p=0.673$; two-way art-ANOVA; Figure $5 \mathrm{G}$ ), indicating a lower fidelity of GABA transmission. Finally, the two synapse types also differed in their kinetic properties. Specifically, GABAergic responses had longer rise times, broader half-widths, and longer decay time constants at CB1-to-PN 
compared to PV-to-PN pairs (Supplementary Figure S2). These results demonstrate that CB1+ INs generally form weaker, less reliable, and less precise GABAergic synapses onto PNs compared to $\mathrm{PV}+\mathrm{INs}$ in neocortex.

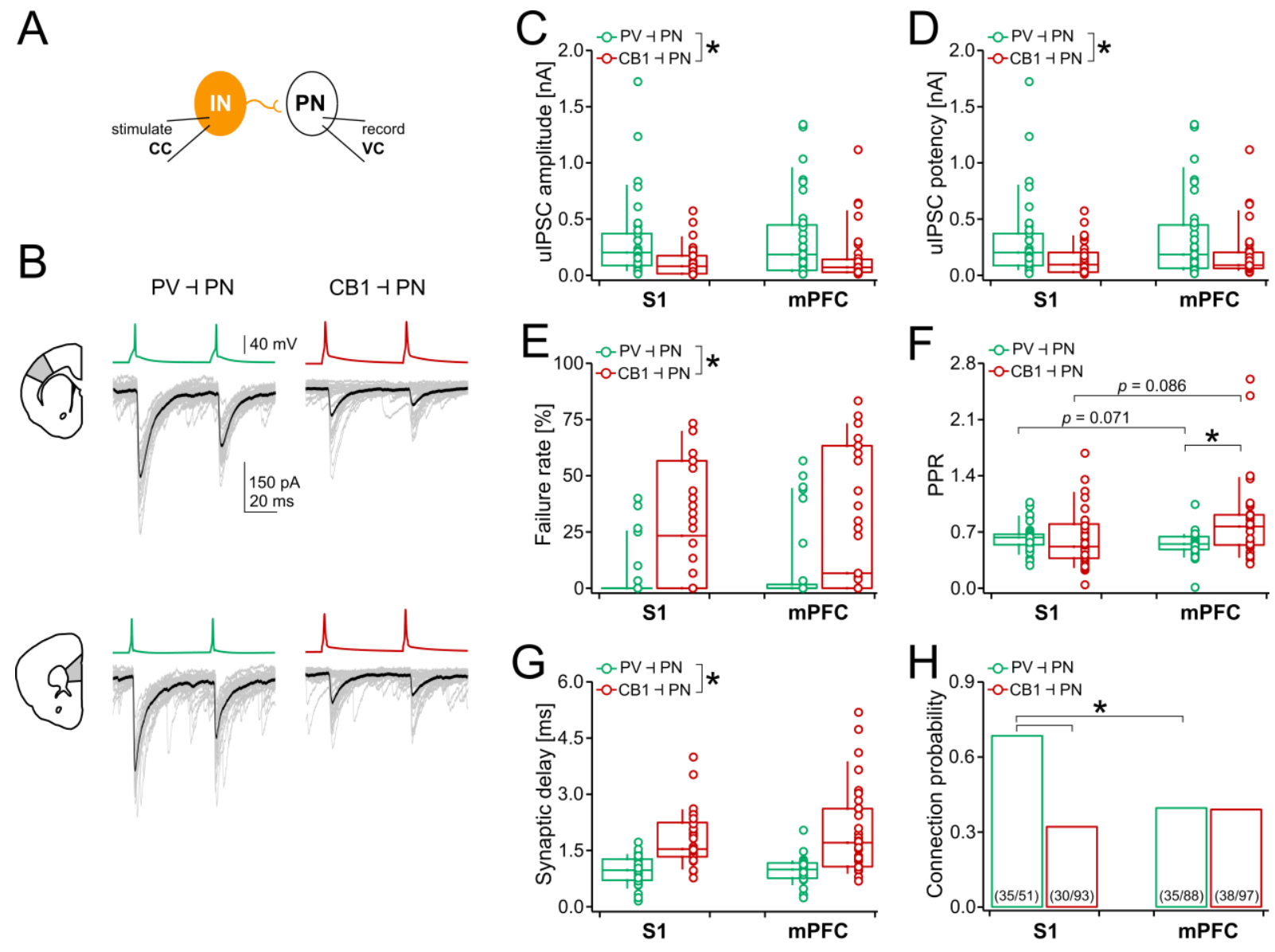

Figure 5: The inhibitory connectivity of $\mathrm{PV}+$ and $\mathrm{CB} 1+$ interneurons converges from somatosensory to prefrontal cortex. A) Illustration of pairwise recording configuration between a presynaptic IN and a postsynaptic PN. Pairwise IN-to-PN recordings performed in S1 and mPFC L2/3 in the presence of the glutamatergic AMPA receptor blocker NBQX $(10 \mu \mathrm{M})$. B) Example traces of synaptically connected PV-to-PN pairs (green) and CB1-toPN pairs (red) in S1 (upper panel) and mPFC (lower panel). C-G) Summary graphs depicting the (C) uIPSC amplitude, (D) uIPSC potency, (E) the failure rate, (F) the PPR, and (G) the synaptic delay of uIPSCs for PV-to-PN and CB1-to-PN pairs in S1 and mPFC. Data are presented as box plots. Whiskers represent the $10^{\text {th }}$ and $90^{\text {th }}$ percentile. Dots represent individual data points. H) Bar graph showing the connection probability of $\mathrm{PV}+$ and $\mathrm{CB} 1+$ INs onto PNs in S1 and mPFC. The number of connected pairs / all pairwise recordings is given in the parentheses. Panels C-G); PV-to-PN pairs: $\mathrm{n} / \mathrm{m}=35 / 15$ for $\mathrm{S} 1, \mathrm{n} / \mathrm{m}=33 / 18$ for $\mathrm{mPFC}$; CB1-to-PN pairs: $\mathrm{n} / \mathrm{m}=30 / 15$ for $\mathrm{S} 1, \mathrm{n} / \mathrm{m}$ $=34 / 18$ for mPFC. Data were analyzed with two-way art-ANOVAs or binary logistic regression followed by post-hoc Bonferroni Holm-adjusted Mann-Whitney U tests or Fisher's exact tests. * $p<0.05$.

An important estimate of inhibitory control is the probability with which different IN types form GABAergic synapses onto PNs. This connection probability serves as a measure of the inhibitory innervation density and can be derived by dividing the number of connected pairs by the number of all pairwise recordings. Importantly, I found that the connection probability strongly converged between the two IN types across regions (cell type: $W=16.57, p<0.001$; region: $W=10.36, p=0.001$; cell type $x$ region: $W=9.74, p=0.002$; binary logistic regression followed by post-hoc Bonferroni-Holm adjusted Fisher's exact tests; Figure 5H). In S1, PV+ INs formed GABAergic synapses onto PNs with a high connection probability of $68.6 \%(35 / 51$ recorded pairs). By contrast, the connection probability of CB1+ INs only reached $32.3 \%$ (30/93 
recorded pairs) and thus was more than twofold lower compared to that of $P V+I N s(p<0.001)$. However, the connection probability was balanced between the two IN types in the mPFC ( $p>0.999)$. This equilibration was largely driven by an almost twofold decrease in the connection probability to $39.8 \%$ for $\mathrm{PV}+\mathrm{INs}$ in the mPFC (35/88 recorded pairs; $p=0.004$ vs. S1). The connection probability of $\mathrm{CB} 1+$ INs was instead largely stable between regions, although a modest, non-significant increase to $39.2 \%$ was seen in the mPFC (38/97 recorded pairs; $p=0.730$; Figure $5 \mathrm{H}$ ). Together, these results demonstrate that while $\mathrm{CB} 1+\mathrm{INs}$ form considerably fewer and generally less powerful GABAergic synapses in S1, their inhibitory connectivity fully converges with that PV+ INs in the mPFC. These findings thus resonate well with the regional differences in the abundance of the two IN types and suggest that a PV+ INdominated inhibitory circuit motif in sensory cortices is replaced by a more symmetrical circuit motif in higher-order associative cortices, in which inhibitory control is largely balanced between $\mathrm{PV}+$ and $\mathrm{CB} 1+\mathrm{INs}$.

\subsubsection{CB1+ interneurons form region-specific subtypes of GABAergic synapses}

Interestingly, I noticed that a fraction of CB1+ INs formed GABAergic synapses with unusually slow kinetics. These slow synapses could be easily distinguished from the majority of fast GABAergic synapses and seemed to be exclusively formed by CB1+ INs in S1 but not mPFC. Consistently, I found that the kinetic parameters of uIPSCs, i.e. the rise time, half width, and decay time constant tau, showed broader distributions and a higher incidence of unusually large values at CB1-to-PN pairs in S1 compared to mPFC (Supplementary Figure S1). These differences usually did not reach significance, possibly due to a high variability. Nonetheless, these observations raised the possibility that CB1+ INs might indeed form different subtypes of GABAergic synapses in different cortical regions.

I therefore tested whether CB1-to-PN pairs can be grouped into different synapse clusters based on their kinetic and other synaptic properties using hierarchical cluster analysis. Of note, the rise times, half-widths, and decay time constants tau were highly intercorrelated (Supplementary Figure S3), which could possibly bias the hierarchical clustering due to overrepresentation of closely related variables. Therefore, I first reduced these kinetic parameters to a single dimension using principal component analysis (Figure 6A; Supplementary Figure S3). The extracted principal component was then fed into a hierarchical cluster analysis together with the uIPSC potency, failure rate, and synaptic delay (Figure 6A). In S1, this approach resulted in a clear two-cluster solution with two highly distinctive clusters of synapses that considerably differed in their signaling kinetics (Figure 6B-D; Supplementary Figure S6). Cluster 1 represented the majority of CB1-to-PN pairs and contained synapses with typical fast synaptic properties, which I therefore classified as fast type-I synapses. In sharp contrast, cluster 2 contained a fraction of synapses that were characterized by remarkably slow and untypical signaling kinetics, as evidenced by their significantly longer rise 
times $(U=-3.66, p<0.001)$, half widths $(U=-4.13, p<0.001)$, and decay time constants tau compared to fast type-I synapses $(U=-4.08, p<0.001$; Mann-Whitney $U$ tests; Figure 6B-D; Supplementary Figure S6). I therefore termed these slow type-II synapses. Besides their signaling kinetics, these slow type-Il synapses also substantially differed from fast type-I synapses in all of their other synaptic properties. Type-Il synapses were generally stronger, as evidenced by a higher uIPSC potency ( $U=-2.35, p=0.019)$. In contrast to type-I synapses, they were further characterized by a complete absence of transmission failures ( $U=-3.66, p<0.001)$. Moreover, slow type-II synapses exclusively exhibited paired-pulse depression and hence showed a reduced PPR compared to fast type-I synapses $(U=-1.97, p=0.049)$. Finally, they also displayed a shorter synaptic delay $(U=-2.02, p=0.044$; Mann-Whitney $U$ tests; Figure $6 \mathrm{~B}$; Supplementary Figure S6). Together, these results suggest that GABAergic synapses made by $\mathrm{CB} 1+$ INs in $\mathrm{S} 1$ can be grouped into fast type-I and slow type-II synapses with highly distinctive synaptic signaling properties.

The same procedure also yielded a two-cluster solution for synapses made by CB1+ INs in the mPFC. However, this solution was less clear, with the resulting synapse clusters being considerably less distinctive compared to those obtained in S1 (Figure 6B). Indeed, the two clusters in mPFC only differed in their failure rates $(U=-4.83, p<0.001)$, reflecting a larger cluster 1 composed of mostly reliable synapses with low failure rates and a smaller cluster 2 containing only unreliable synapses with high failure rates (Figure 6B, C). Besides this, their synaptic properties were largely comparable (uIPSC potency: $U=-1.68, p=0.094$; PPR: $U=-$ $1.80, p=0.073$; synaptic delay: $U=-1.93, p=0.053)$. Most importantly, the two clusters generally displayed fast and highly similar signaling kinetics (rise time: $U=-0.28, p=0.782$; half width: $U=-$ 0.57, $p=0.568$; decay tau: $U=-0.28, p=0.782$; Mann-Whitney $U$ tests; Figure 6B-D; Supplementary Figure S6). Thus, synapses made by CB1+ INs in mPFC seemed to represent fast type-I synapses only and were therefore clustered mainly according to their failure rates as their most prominent distinguishing feature. Only a small fraction of synapses in cluster 1 showed rather high values for the kinetic parameters and thus possibly slow signaling kinetics (Figure 6B). However, these synapses also displayed very small uIPSC amplitudes, which generally complicates the isolation of kinetic parameters from background noise even after averaging, particularly in recordings with high spontaneous activity. Thus, while synapses made by $\mathrm{CB} 1+$ INs could be grouped into highly distinct fast type-I and slow type-II synapses in $\mathrm{S} 1$, no distinctive clusters emerged in the mPFC, presumably due to the presence of fast type-I synapses only. Interestingly, these two synapse types were formed by both regular- and irregular-firing CB1+ INs (Supplementary Figure S7) and were not only region- but also cell type-specific, with no distinctive synapse clusters resulting for PV-to-PN pairs in both cortical regions (Supplementary Figures S5-S6). Indeed, synapses made by PV+ INs almost exclusively showed very fast signaling kinetics (Supplementary Figure S2, S6) and always 
clustered into a large population of strong, highly reliable synapses and a minor fraction of extremely weak and unreliable synapses (Supplementary Figures S5, S6). These clusters therefore strongly resembled those formed by CB1+ INs in the mPFC. Together, these results suggest that CB1+ but not PV+ INs form different subtypes of GABAergic synapses in sensory but not higher-order associative cortices.

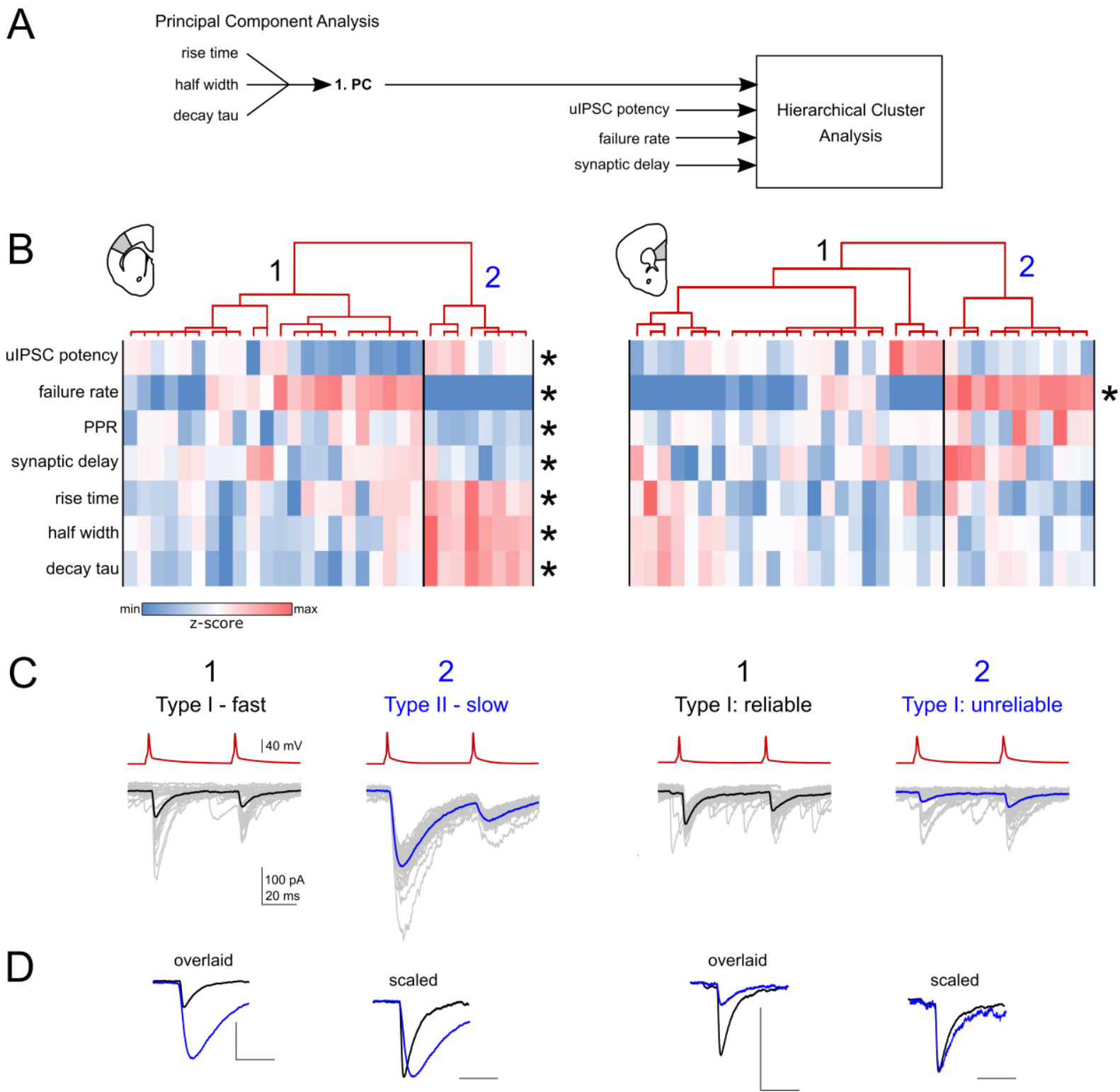

Figure 6: $\mathrm{CB} 1+$ interneurons form distinct subtypes of GABAergic synapses in somatosensory but not prefrontal cortex. A) Illustration of analysis strategy. The kinetic parameters of uIPSCs at connected CB1-to-PN pairs, i.e. the rise time, half width, and decay time constant tau, were first condensed into a single principle component (1. PC) using principal component analysis. This 1. PC was next fed into a hierarchical cluster analysis together with the uIPSC potency, the failure rate, and the synaptic delay. B) Two-cluster solutions for synapses made by CB1+ INs in S1 (left) and mPFC (right). Upper part: Dendrograms. The two clusters are denoted by 1 and 2. Lower part: Heat maps depicting the synaptic properties of individual CB1-to-PN pairs from clusters 1 and 2. Synaptic parameters were $z$-transformed and then color-coded, with blue representing minimum and red colors representing maximum values. C) Example traces of connected CB1-to-PN pairs from cluster 1 and 2 in $\mathrm{S} 1$ and mPFC. D) Average uIPSCs recorded in PNs in response to the first presynaptic action potential at CB1-to-PN pairs shown in C), overlaid and scaled between cluster 1 (black) and cluster 2 (blue). Synapses made by CB1+ INs in S1 were categorized into fast type-I (cluster 1) and slow type-II (cluster 2) synapses. Synapses made by CB1+ INs in mPFC were generally fast type-l, with a sub-clustering into reliable (cluster 1) and unreliable (cluster 2) synapses. S1: $n / m=22 / 14$ for cluster $1, n / m=8 / 5$ for cluster 2 ; $m$ PFC: $n / m=23 / 16$ for cluster $1, n / m=11 / 7$ for cluster 2 . Hierarchical cluster analysis was performed using Ward fusion algorithm and squared Euclidian distance as linkage distance. Clusters were compared with Mann-Whitney U tests. * $p<0.05$. 


\subsection{Prefrontal inhibitory networks formed by $\mathrm{CB} 1+$ and $\mathrm{PV}+$ interneurons reach functional maturity early in development}

Neocortical circuits undergo a prolonged developmental process of experience-dependent network remodeling in order to reach their functional maturity, commonly referred to as critical period plasticity (Hensch, 2005). In sensory systems, the onset of such critical period plasticity is believed to be largely driven by the maturation of PV+ INs (Hensch, 2005). Consistent with this, I found that they represent an abundant and dominant IN type in these regions. However, my results also showed that a different network motif operates at the level of the prefrontal cortex, where inhibitory control is largely balanced between $\mathrm{PV}+$ and $\mathrm{CB} 1+\mathrm{INs}$, raising the possibility that developmental circuit remodeling in higher-order associative cortices is driven not only by PV+ INs but rather the concerted activity of both IN types. Prefrontal critical period plasticity is assumed to occur during adolescence, a transitional phase between the juvenile stage and early adulthood (Spear, 2000; Caballero and Tseng, 2016). I therefore sought to characterize the developmental trajectories of inhibitory networks made by CB1+ and PV+ INs in prefrontal cortex during the adolescent phase.

\subsubsection{GABAergic synapses of prefrontal CB1 + and PV + interneurons are formed early in development}

I first assessed whether GABAergic synapses made by PV+ and CB1+ INs in prefrontal cortex undergo functional developmental changes during adolescence. In rats and mice, the adolescent phase roughly spans from P30-45 (Spear, 2000). Therefore, I performed pairwise patch-clamp recordings in mPFC L2/3 before and after on P20-30 and P50-70, i.e. during the juvenile stage and early adulthood (Figure 7A).

Remarkably, I found that inhibitory connectivity patterns of both prefrontal CB1+ and PV+ INs were highly stable between the two age points. For instance, there were no developmental changes in the connection probability of the two IN types (PV: 36.4\% [16/44] for P20-30, 37.1\% [23/62] for P50-70; CB1: 34.7\% [17/49] for P20-30; 43.4\% [36/83] for P50-70; age: $W=0.01$, $p=0.939$; cell type: $W=0.03, p=0.867$; age $x$ cell type: $W=0.37, p=0.546$; binary logistic regression; Figure 7D). This suggests that $\mathrm{CB} 1+$ and $\mathrm{PV}+\mathrm{INs}$ formed most, if not all, their GABAergic synapses onto PNs already by P20-30, i.e. during the juvenile stage. Moreover, these synapses already displayed typical adult-like properties, with only some modest nonsignificant developmental differences (Figure 7B). Descriptively, GABAergic signaling at both CB1-to-PN and PV-to-PN pairs appeared slightly weaker at P20-30 compared to P50-70, as evidenced by their uIPSC amplitudes and potencies (Figure 7B, E, F). However, these differences were only modest and non-significant (age: $F_{(1,87)}=0.04, p=0.840$; age $x$ cell type: $F_{(1,87)}=0.38, p=0.541$; for ulPSC amplitude; age: $F_{(1,87)}=0.01, p=0.905$; age $x$ cell type: $F_{(1,87)}=0.63, p=0.430$; for uIPSC potency; two-way art-ANOVA; $\mathrm{n} / \mathrm{m}=16-35$ connected pairs from 6-17 mice per cell type / age point). Interestingly, the uIPSC amplitudes and potencies 
also did not substantially differ between CB1-to-PN and PV-to-PN pairs in general (cell type: $F_{(1,87)}=3.08, p=0.083$; for uIPSC amplitude; $F_{(1,87)}=0.80, p=0.373$; for uIPSC potency; Figure $7 \mathrm{E})$, indicating that their synaptic strength is largely comparable. In line with my previous findings, synapses made by CB1+ but not PV+ INs were characterized by high failure rates and this pattern was highly stable throughout development (age: $F_{(1,87)}=0.73, p=0.397$; cell type: $F_{(1,87)}=21.01, p<0.001$; age $x$ cell type: $F_{(1,87)}=0.30, p=0.588$; two-way art-ANOVA; Figure 7G). Consistently, uIPSCs at CB1-to-PN pairs showed a higher PPR compared to those at PVto-PN pairs across development, indicating a lower Pr at synapses made by CB1+ INs (age: $F_{(1,87)}=0.27, p=0.602$; cell type: $F_{(1,87)}=22.78, p<0.001$; age $x$ cell type: $F_{(1,87)}=0.84, p=0.363$; two-way art-ANOVA; data not shown). Together, these results show that GABAergic synapses made by $\mathrm{CB} 1+$ and $\mathrm{PV}+\mathrm{INs}$ in the MPFC reach functional maturity already during the juvenile stage and thus before adolescence.

A
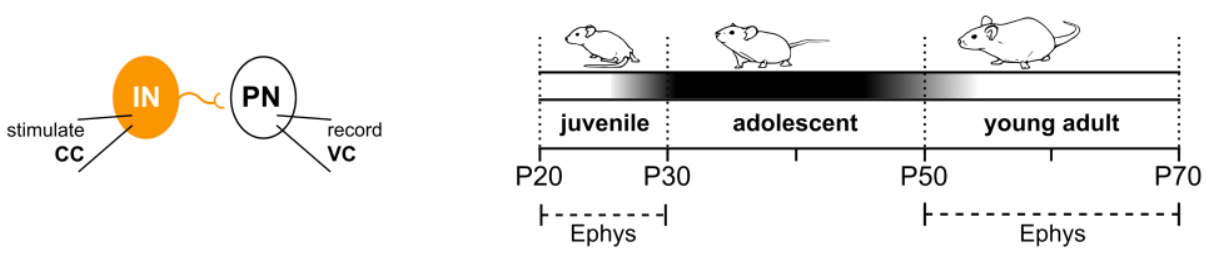

B
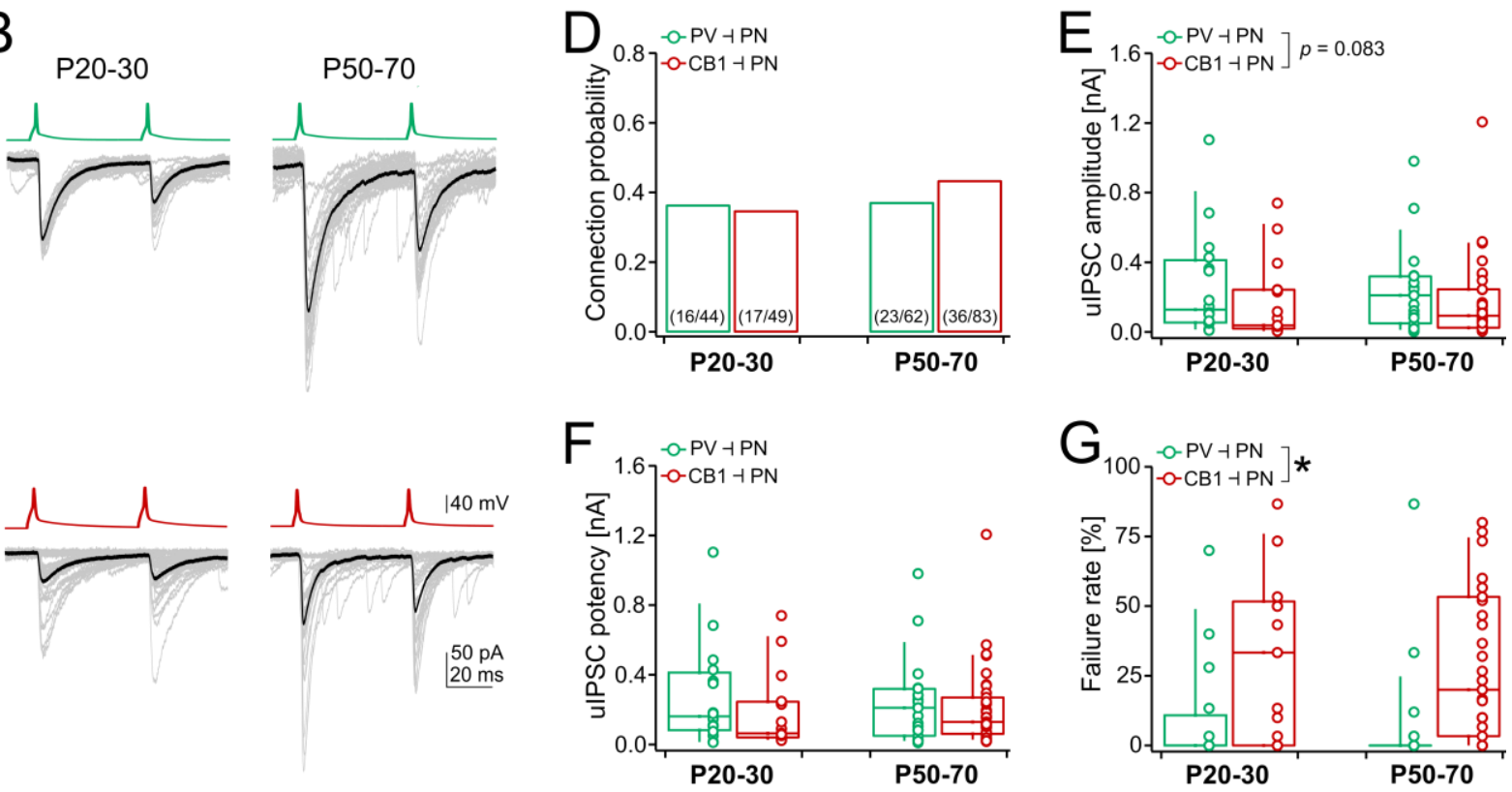

Figure 7: $\mathrm{CB1}+$ and PV+ interneurons form functional GABAergic synapses onto pyramidal neurons in prefrontal cortex before the adolescent phase. A) Illustration of experimental protocol. Pairwise IN-to-PN recordings were conducted between P20-30 (juvenile stage) or between P50-70 (young adulthood), i.e. before and after the adolescent phase. Recordings were made in mPFC L2/3 in the presence of the glutamatergic AMPA receptor blocker NBQX $(10 \mu \mathrm{M})$. B) Example traces of synaptically connected PV-to-PN pairs (green, upper panel) and CB1-to-PN pairs (red, lower panel) at P20-30 (left) and P50-70 (right). D) Bar graph showing the connection probability of PV+ and CB1+ INs onto PNs in S1 and mPFC. The number of connected pairs / all painwise recordings is given in the parentheses. E-F) Summary graphs depicting (E) the uIPSC amplitude, (F) the uIPSC potency, and (G) the failure rate for PV-to-PN and CB1-to-PN pairs at P20-30 and P50-70. Data are presented as box plots. Whiskers represent the $10^{\text {th }}$ and $90^{\text {th }}$ percentile. Dots represent individual data points. PV-to-PN pairs: $\mathrm{n} / \mathrm{m}=16 / 6$ for P20-30, $n / m=23 / 12$ for P50-70; CB1-to-PN pairs: $n / m=17 / 7$ for P20-30, $n / m=35 / 17$ for P50-70. Data were analyzed with two-way art-ANOVAs or binary logistic regression followed by post-hoc Bonferroni Holm-adjusted Mann-Whitney U tests or Fisher's exact tests. * $p<0.05$. 


\subsubsection{Glutamatergic inputs onto prefrontal $P V+$ but not $C B 1+$ interneurons undergo synaptic remodeling during development}

The functionality of inhibitory networks also depends on their glutamatergic excitatory inputs. Therefore, I next assessed whether the excitatory input synapses onto prefrontal PV+ and CB1+ INs undergo some form of synaptic remodeling during adolescence. To this end, I recorded mEPSCs in the two IN types in mPFC L2/3 in the presence of the voltage-gated sodium-channel blocker TTX $(1 \mu \mathrm{M})$ at selected time points before, during, and after the adolescent phase, i.e. P21, P35, and P56 (Figure 8A).

I found prominent differences in mEPSCs between the two IN types, but only modest developmental changes. PV+ INs generally displayed a two- to threefold higher mEPSC frequency compared to $\mathrm{CB} 1+\mathrm{INs}$, indicating that they receive more glutamatergic inputs (Figure 8B-C). Specifically, mEPSCs occurred with considerably shorter inter-event intervals in PV + compared to CB1 + INs at all developmental time points, as reflected by their cumulative distributions (fraction: $F_{(1.07,110.42)}=129.42, p<0.001$; fraction $x$ cell type: $F_{(1.07,110.42)}=44.12$, $p<0.001$; fraction $x$ age: $F_{(2.14,110.42)}=0.33, p=0.737$; fraction $\times$ cell type $\times$ age: $F_{(2.14,110.42)}=0.46$, $p=0.646$; three-way $\mathrm{rm}$-ANOVA with fraction as within-subject factor; $\mathrm{n} / \mathrm{m}=16$-20 cells from 45 mice per cell type / age; Figure $8 \mathrm{C}$ ). This pattern was largely stable across development, with only modest cell type-specific changes in the mEPSC frequency being evident (age: $F_{(2,103)}=3.55, p=0.032$; cell type: $F_{(1,103)}=126.85, p<0.001$; age $\times$ cell type: $F_{(2,103)}=6.85, p=0.002$; two-way ANOVA; Figure 8D). While there were no developmental changes in CB1+ INs (age: $F_{(2,56)}=0.54, p=0.59$; one-way ANOVA), I observed a modest decrease in mEPSC frequency in PV+ INs from P21 to P35 (age: $F_{(2,47)}=6.07, p=0.005 ; \mathrm{P} 21$ vs. P35: $p=0.003$; P21 vs. P56: $p=0.012$; P35 vs. P56: $p>0.999$; one-way ANOVA followed by post-hoc Bonferroni-Holm adjusted unpaired $t$-tests). These data suggest that the number of and/or spontaneous vesicle fusion propensity at functional AMPA receptor-positive glutamatergic input synapses onto PV+ but not CB1+ INs decreases towards adolescence.

$\mathrm{PV}+\mathrm{INs}$ further exhibited a higher mEPSC amplitude compared to CB1+ INs at all age points. This was reflected in their right-shifted cumulative amplitude distributions, suggesting that they also receive stronger glutamatergic inputs compared to $C B 1+I N s$ (fraction: $F_{(1.27,130.44)}=740.30$, $p<0.001$; fraction $x$ cell type: $F_{(1.27,130.44)}=13.04, p<0.001$; fraction $x$ age: $F_{(2.53,130.44)}=0.49$, $p=0.661$; fraction $x$ cell type $x$ age: $F_{(2.53,130.44)}=1.07, p=0.358$; three-way rm-ANOVA; Figure $8 \mathrm{E})$. Again, this pattern was highly stable across development, with only small cell typeindependent changes being evident (age: $F_{(2,103)}=7.61, p=0.001$; cell type: $F_{(1,103)}=38.03$, $p<0.001$; age $x$ cell type: $F_{(2,103)}=0.41, p=0.665$; two-way ANOVA; Figure $\left.8 F\right)$. Specifically, the mEPSC amplitude slightly decreased between P21 and P56 in both IN types (P21 vs. P35: $p=0.156$; P21 vs. P56: $p<0.001$; P35 vs. P56: $p=0.102$; post-hoc Bonferroni-Holm adjusted unpaired $t$-tests). The decrease in the mEPSC amplitude may indicate a decline in postsynaptic 
AMPA receptor content and thus a weakening of glutamatergic synapses during development. The developmental changes in the mEPSC frequency in PV+ INs may be partly driven by changes in the spontaneous presynaptic vesicle fusion propensity. However, a more likely mechanism is developmental pruning of excitatory synapses, as shown for PV+ INs in the adolescent monkey prefrontal cortex (Chung et al., 2017). Together, these results indicate that excitatory synapses onto prefrontal INs undergo a form of remodeling during adolescence that is mainly driven by synapse elimination and weakening. However, these changes are only modest and mostly restricted to PV+ INs.

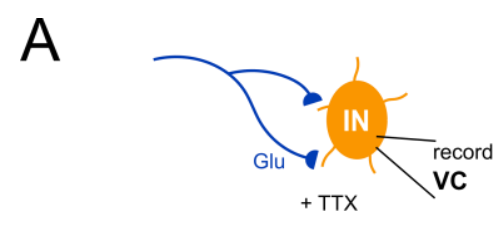

$\mathrm{B}$

PV

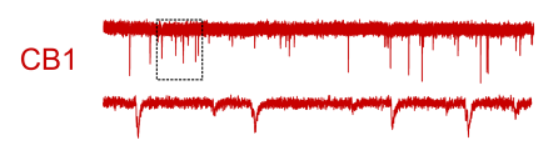

C
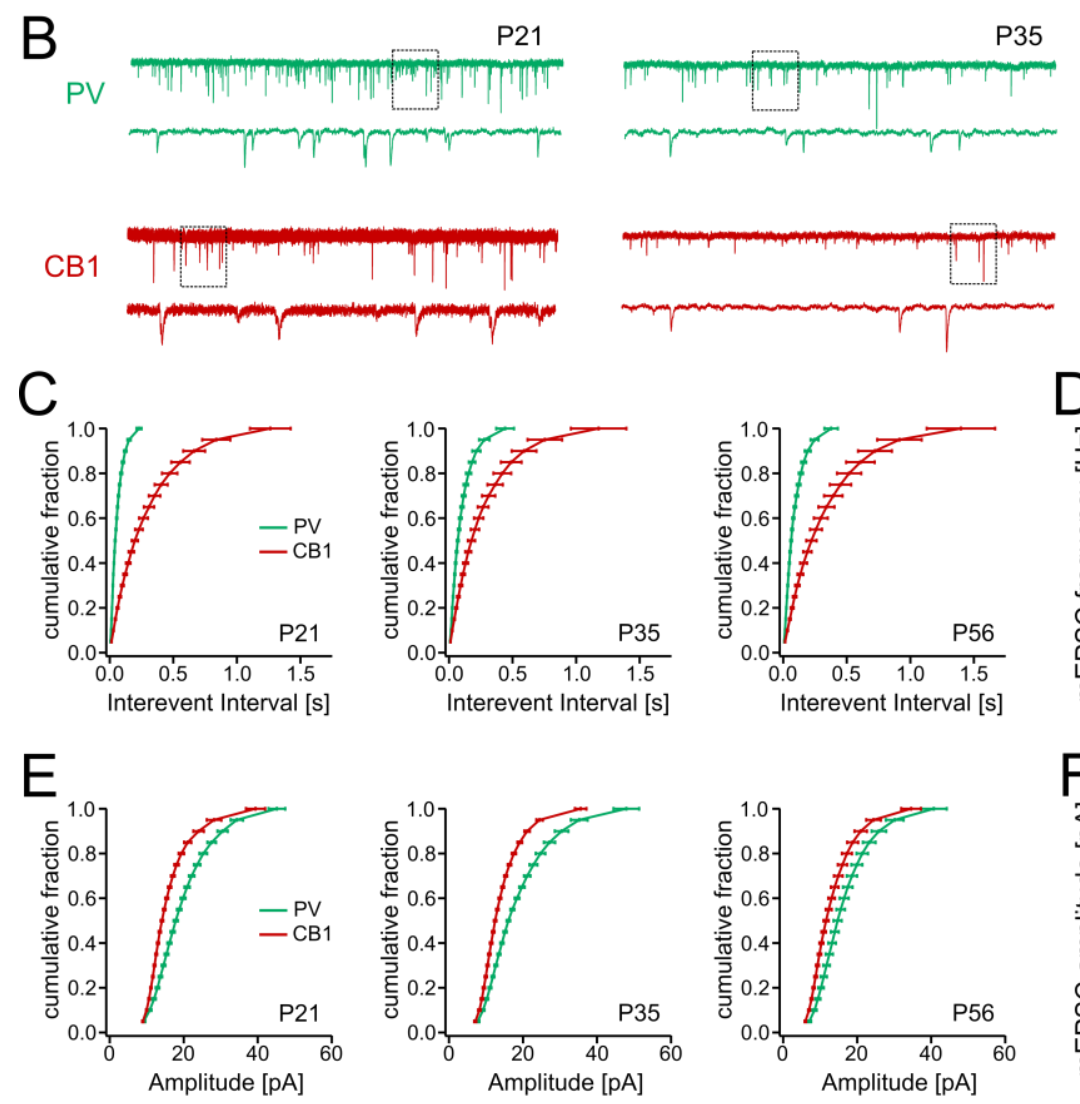
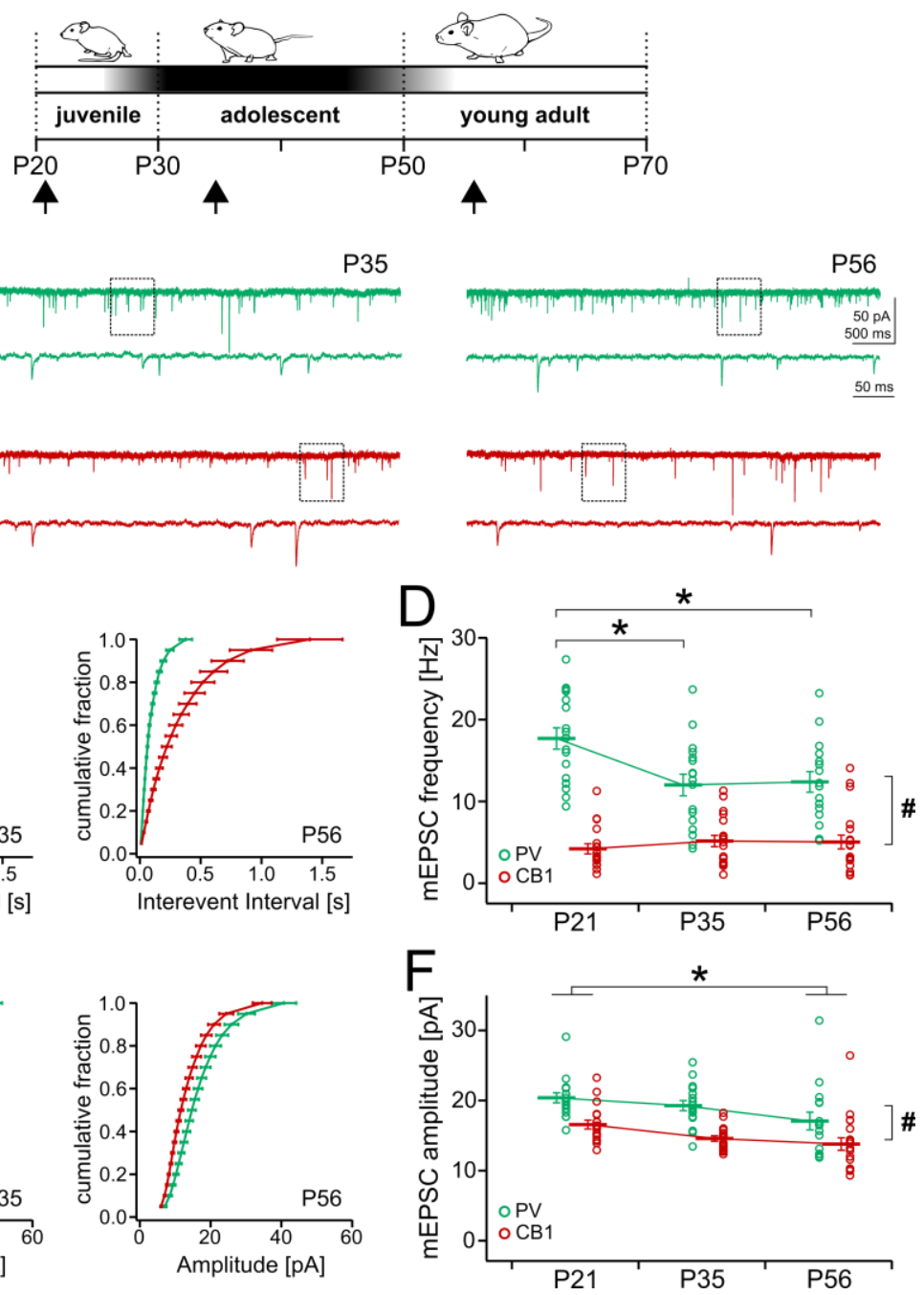

Figure 8: Developmental changes in mEPSCs during adolescence are more prominent in PV+ than in CB1+ interneurons in prefrontal cortex. A) Illustration of experimental protocol. An IN was held at $-70 \mathrm{mV}$ in voltageclamp (VC) mode and glutamatergic mEPSCs were recorded in the presence of the voltage-gated sodium channel blocker TTX $(1 \mu \mathrm{M})$. GABAergic transmission was blocked with PTX $(50 \mu \mathrm{M})$. Recordings were made in mPFC L2/3 at three developmental time points (indicated by arrows): P21 \pm 1 (juvenile stage), P $35 \pm 2$ (adolescent phase), and P56 \pm 2 (young adulthood). B) Example mEPSC traces recorded in PV+ INs (green; upper panel) and CB1+ INs (red; lower panel) at P21, P35, and P56. Shown are $4 \mathrm{~s}$ traces, together with an enlarged $500 \mathrm{~ms}$ segment below the original trace (indicated by the dotted boxes). C) Cumulative probability distributions for mEPSC inter-event intervals for PV+ and CB1+ INs at P21, P35, and P56. D) Summary graph depicting the mEPSC frequency at the three different age points for PV+ and CB1+ INs. E) Cumulative probability distributions for mEPSC amplitudes for PV+ and CB1+ INs at P21, P35, and P56. F) Summary graph depicting the mEPSC amplitude at the three different age points for $\mathrm{PV}+$ and $\mathrm{CB} 1+\mathrm{INs}$. Data are presented as mean $\pm \mathrm{SEM}$. Dots in $\mathbf{D}$ and $\mathbf{F}$ represent individual data points. PV+ INs: $n / m=17 / 4$ for P21, $n / m=17 / 5$ for for P35, $n / m=16 / 5$ for P56; CB1+ INs: $n / m=20 / 4$ for P21, $n / m$ $=19 / 5$ for P35, $\mathrm{n} / \mathrm{m}=20 / 5$ for P56. Data were analyzed with two- or one-way rm-ANOVAs followed by post-hoc Bonferroni Holm-adjusted unpaired $t$-tests. ${ }^{*} p<0.05$. 


\subsection{Prefrontal inhibitory networks formed by $\mathrm{CB} 1+$ but not PV+ interneurons show specific changes in response to developmental stress}

The prefrontal cortex is highly susceptible to the adverse effects of chronic stress. This stress vulnerability is particularly pronounced during adolescence and is assumed to contribute to the development of stress-related neurodevelopmental disorders, such as schizophrenia and major depression (Caballero and Tseng, 2016). Exposure to stress or stress hormones directly, such as the CORT, alters eCB signaling in the prefrontal cortex (e.g. Hill et al., 2011b; Gray et al., 2016), indicating that chronic glucocorticoid-mediated stress might exert its effects at least partly by dysregulating GABAergic transmission at synapses made by CB1+ INs. I therefore sought to determine whether chronic stress during adolescence affects the functionality of prefrontal inhibitory networks formed by CB1+ or PV+ INs. I induced stress via chronic administration of CORT through the drinking water, which has previously been shown to trigger robust anxiety- and depression-related phenotypes in mice (Karatsoreos et al., 2010; Moda-Sava et al., 2019).

\subsubsection{Chronic corticosterone treatment induces a stress-related phenotype}

I first validated whether chronic CORT treatment during adolescence induces stress-related physiological and behavioral alterations. Male and female mice were treated with CORT $(0.1$ $\mathrm{mg} / \mathrm{ml}$ ) or vehicle $(\mathrm{VEH} ; 1 \% \mathrm{EtOH})$ through the drinking water for a duration of 20 days from P30 to P50 (Figure 9A).

Consistent with a stress-related phenotype, mice chronically treated with CORT gained less weight during the treatment phase. This effect was especially pronounced in male mice (age: $F_{(2.97,47.57)}=302.19, p<0.001$; age $x$ drug: $F_{(2.97,47.57)}=19.73, p<0.001$; two-way rm-ANOVA; $m=9$ for CORT, $m=9$ for VEH; Figure 9B). Specifically, CORT-treated males showed a strongly delayed weight gain and thus exhibited a lower body weight compared to $\mathrm{VEH}$-treated controls at all age points after the beginning of the treatment protocol (P30: $p>0.999$, P35: $p=0.003$, P40: $p=0.001$, P45: $p=0.002$, P50: $p=0.012$; post-hoc Bonferroni-Holm adjusted unpaired $t$ tests). Such a delayed weight gain was also seen in CORT-treated females, but the effect was only transient (age: $F_{(2.17,43.29)}=153.75, p<0.001$; age $x$ drug: $F_{(2.17,43.29)}=18.81, p<0.001$; twoway rm-ANOVA; $m=9$ for CORT, $m=13$ for VEH; Figure $9 \mathrm{C})$. Specifically, CORT-treated females showed a lower body weight compared to VEH controls at P35 and P40, but reached a similar body weight by P45 (P30: $p=0.600$, P35: $p=0.024, \mathrm{P} 40: p=0.001, \mathrm{P} 45: p=0.225, \mathrm{P} 50$ : $p>0.999$; post-hoc Bonferroni-Holm adjusted unpaired $t$-tests).

At young adulthood (P50), I further tested possible CORT-induced changes in anxiety-related behavior using the open field test (Figure 9A). I found that CORT-treated mice spent significantly less time in the center of the open field compared to VEH controls, indicating increased anxiety-like behavior (drug: $F_{(1,36)}=7.75, p=0.008$; Figure 9D-E). Descriptively, these changes were more pronounced in females than in males, but the effect was not significant 
(drug $x$ sex: $\left.F_{(1,36)}=2.46, p=0.125\right)$. Moreover, females generally spent less time in the center compared to males, irrespective of drug treatment (sex: $F_{(1,36)}=6.69, p=0.014$; two-way ANOVA; $m=9-13$ per drug treatment / sex). Importantly, there were no differences in the distance traveled (drug: $F_{(1,36)}=1.28, p=0.265$; sex: $F_{(1,36)}=2.20, p=0.147$; drug $x$ sex: $F_{(1,36)}=0.72, p=0.400$; two-way ANOVA; Figure 9F), ruling out that CORT effects on center time were secondary to changes in locomotor activity. Together, these results confirm that chronic CORT treatment during adolescence leads to sex-dependent, stress-related physiological and behavioral abnormalities.

A

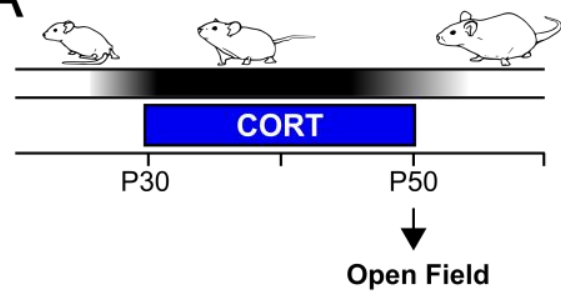

$\mathrm{D}$
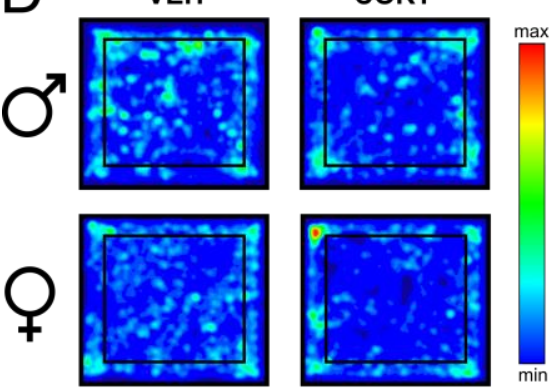

$B$
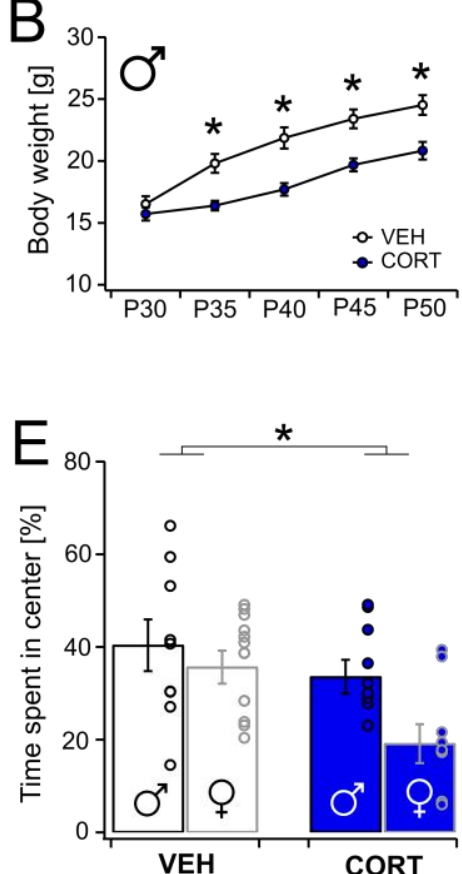
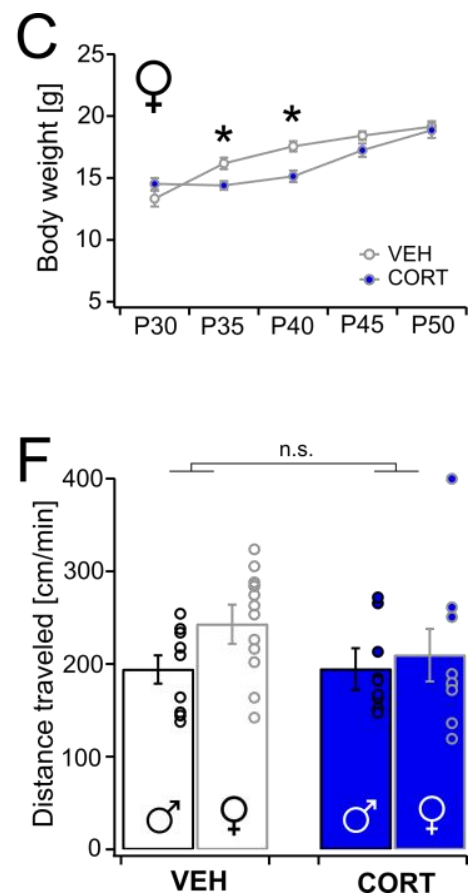

Figure 9: Chronic corticosterone treatment induces stress-related physiological and behavioral changes. A) Illustration of experimental protocol. Mice received CORT $(0.1 \mathrm{mg} / \mathrm{ml}$ through the drinking water) for 20 days from P30 throughout adolescence and were subjected to an open field test at P50. VEH-treated controls received $1 \%$ EtOH. B-C) Growth curves depicting the change in body weight over development in VEH- (white fill) and CORTtreated mice (blue fill) for (B) males (black line color) and (C) females (gray line color). D) Representative heat maps depicting the relative time spent in different locations of the open field for a VEH- and CORT-treated male and female. The open field center is indicated by the black line. Blue represent minimum and red colors maximum values. E-F) Summary graphs showing (E) the time spent in the center of the open field and (F) the distance traveled for VEH- and CORT-treated males and females. Data are presented as mean \pm SEM. VEH males: $m=9$; VEH females: $m=13$; CORT males: $m=9$; CORT females: $m=9$; Dots in $E$ and $\mathbf{F}$ represent individual data points. Data were analyzed with two-way rm-ANOVAs or two-way ANOVAs followed by post-hoc Bonferroni Holm-adjusted unpaired $t$-tests. * $p<0.05$.

\subsubsection{Chronic corticosterone treatment does not affect GABAergic synapses made by $\mathrm{PV}+$ interneurons in prefrontal cortex}

I next assessed whether chronic CORT treatment during adolescence induces alterations at GABAergic synapses made by PV+ INs in prefrontal cortex. To this end, mice were chronically treated with CORT or vehicle from P30 for at least 20 days. I then performed pairwise recordings between PV+ INs and PNs in mPFC L2/3 between P50-70, with drug treatment being continued throughout this period (Figure 10A). 
A

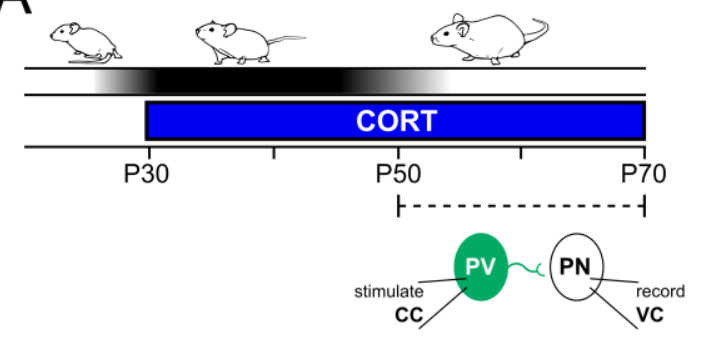

B
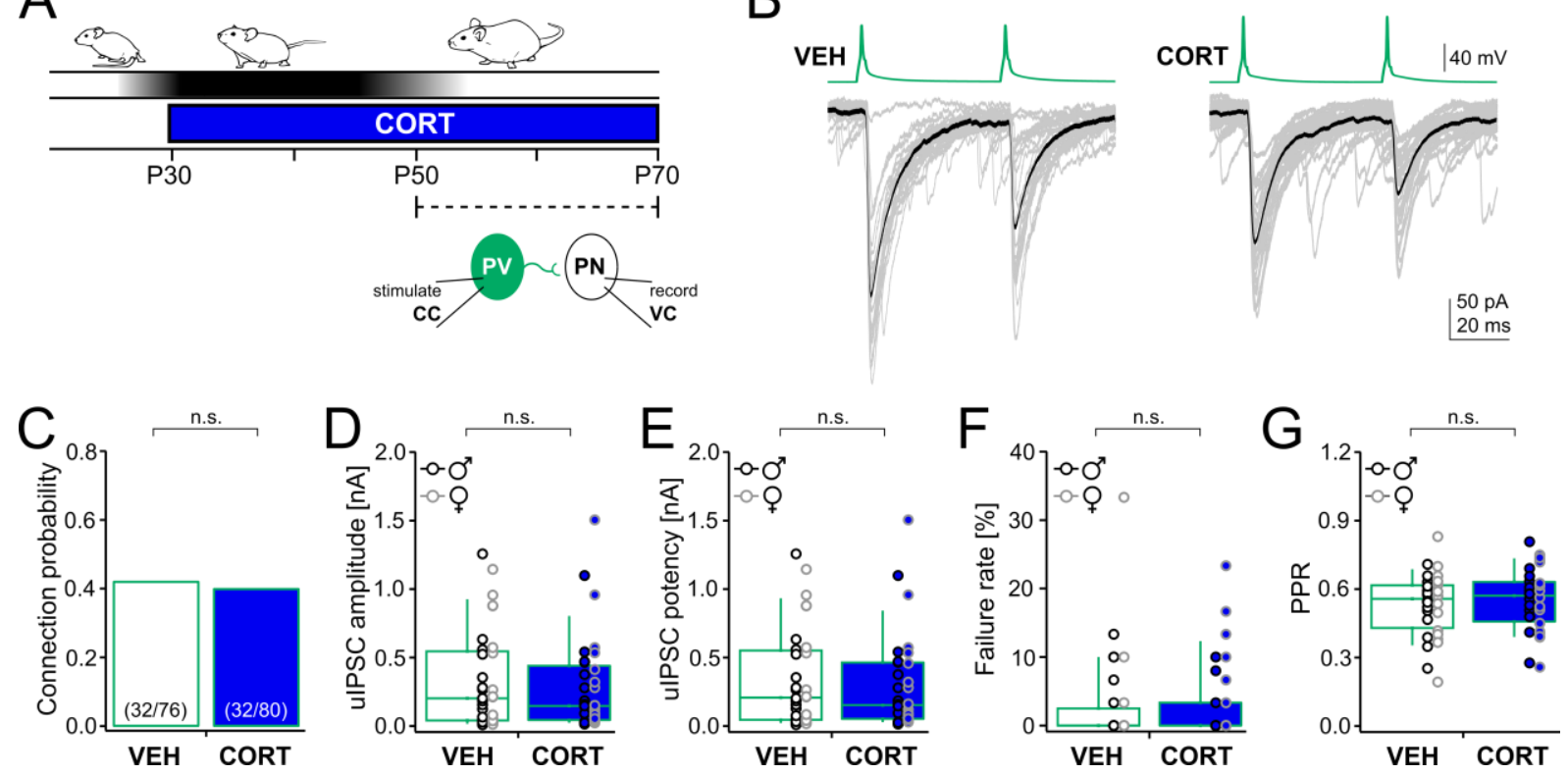

Figure 10: Chronic corticosterone treatment does not affect synaptic transmission at GABAergic synapses made by $\mathbf{P V}+$ interneurons in prefrontal cortex. A) Illustration of experimental protocol. Mice received CORT $(0.1 \mathrm{mg} / \mathrm{ml}$ through the drinking water) for at least 20 days from P30 throughout adolescence, followed by pairwise PV-to-PN recordings in MPFC L2/3 between P50-70 under continued drug treatment. VEH-treated controls received $1 \% \mathrm{EtOH}$. Pairwise recordings were made in the presence of the glutamatergic AMPA receptor blocker NBQX (10 $\mu \mathrm{M})$. B) Example traces of synaptically connected PV-to-PN pairs from a VEH- and CORT-treated mouse. C) Bar graph showing the connection probability of PV+ INs onto PNs for VEH- and CORT-treated mice. The number of connected pairs / all pairwise recordings is given in the parentheses. D-G) Summary graphs depicting (D) the uIPSC amplitude, (E) the uIPSC potency, (F) the failure rate, and (G) the PPR for connected PV-to-PN pairs obtained from VEH (white) and CORT-treated mice (blue). Data are presented as box plots. Whiskers represent the $10^{\text {th }}$ and $90^{\text {th }}$ percentile. Dots represent individual data points, with males highlighted in black and females highlighted in gray. VEH: $n / m=16 / 8$ for males, $n / m=16 / 6$ for females; CORT: $n / m=15 / 7$ for males, $n / m=17 / 8$ for females. Data were analyzed with Fisher's exact test and two-way art-ANOVAs. ${ }^{*} p<0.05$.

I found that GABAergic synapses made by PV+ INs remained largely unaffected by CORT treatment. There were no observable differences in the connection probability of PV+ INs between CORT-treated mice (40.0\% [32/80 recorded pairs]) and VEH controls (42.1\% [32/76 recorded pairs]; $X^{2}=0.07, p=0.871$; Fisher's exact test; Figure $10 \mathrm{C}$ ), indicating that chronic CORT treatment does not affect the number of GABAergic synapses made by PV+ INs. Moreover, synapses made by PV+ INs in CORT-treated mice were highly comparable in their properties to those seen in VEH-treated mice (Figure 10B, D-G). In both sexes, chronic CORT treatment neither affected the uIPSC amplitude (drug: $F_{(1,60)}=0.10, p=0.753$; sex: $F_{(1,60)}=0.01$, $p=0.946$; drug $x$ sex: $F_{(1,60)}=0.01, p=0.939$; two-way art-ANOVA; $\mathrm{n} / \mathrm{m}=15-17$ connected pairs from 6-8 mice per drug treatment / sex; Figure 10D) nor the uIPSC potency (drug: $F_{(1,60)}=0.08$, $p=0.783$; sex: $F_{(1,60)}=0.01, p=0.926$; drug $x$ sex: $F_{(1,60)}=0.00, p=0.972$; two-way art-ANOVA; Figure 10E). There were also no effects on the failure rate (drug: $F_{(1,60)}=0.80, p=0.376$; sex: $F_{(1,60)}=0.90, p=0.346$; drug $\times$ sex: $F_{(1,60)}=2.47, p=0.121$; two-way art-ANOVA; Figure 10F) or the PPR (drug: $F_{(1,60)}=0.35, p=0.577$; sex: $F_{(1,60)}=0.00, p=0.998$; drug x sex: $F_{(1,60)}=0.03, p=0.871$; two-way art-ANOVA; Figure 10G). Together, these results demonstrate that GABAergic synapses made by PV+ INs in the mPFC are highly resilient against the effects of CORT treatment during adolescence. 


\subsubsection{Chronic corticosterone treatment disrupts endocannabinoid signaling at} GABAergic synapses made by CB1+ interneurons in prefrontal cortex

Using the same experimental protocol, I further examined whether chronic CORT treatment during adolescence leads to functional alterations at GABAergic synapses made by CB1+ INs in mPFC L2/3 (Figure 11A). Of note, CB1-to-PN recordings were carried out in the same mice as used for the PV-to-PN recordings.

A

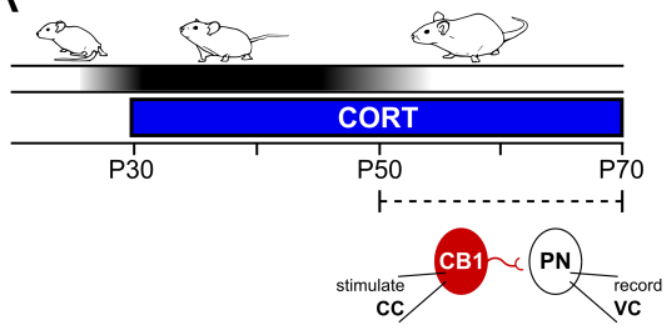

B

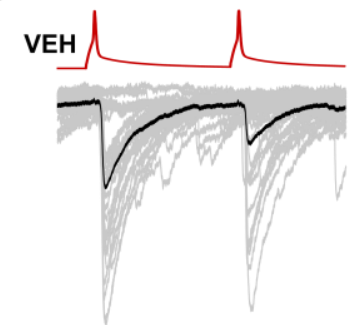

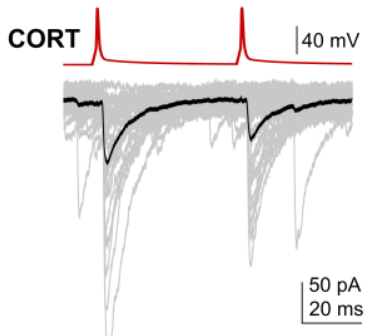
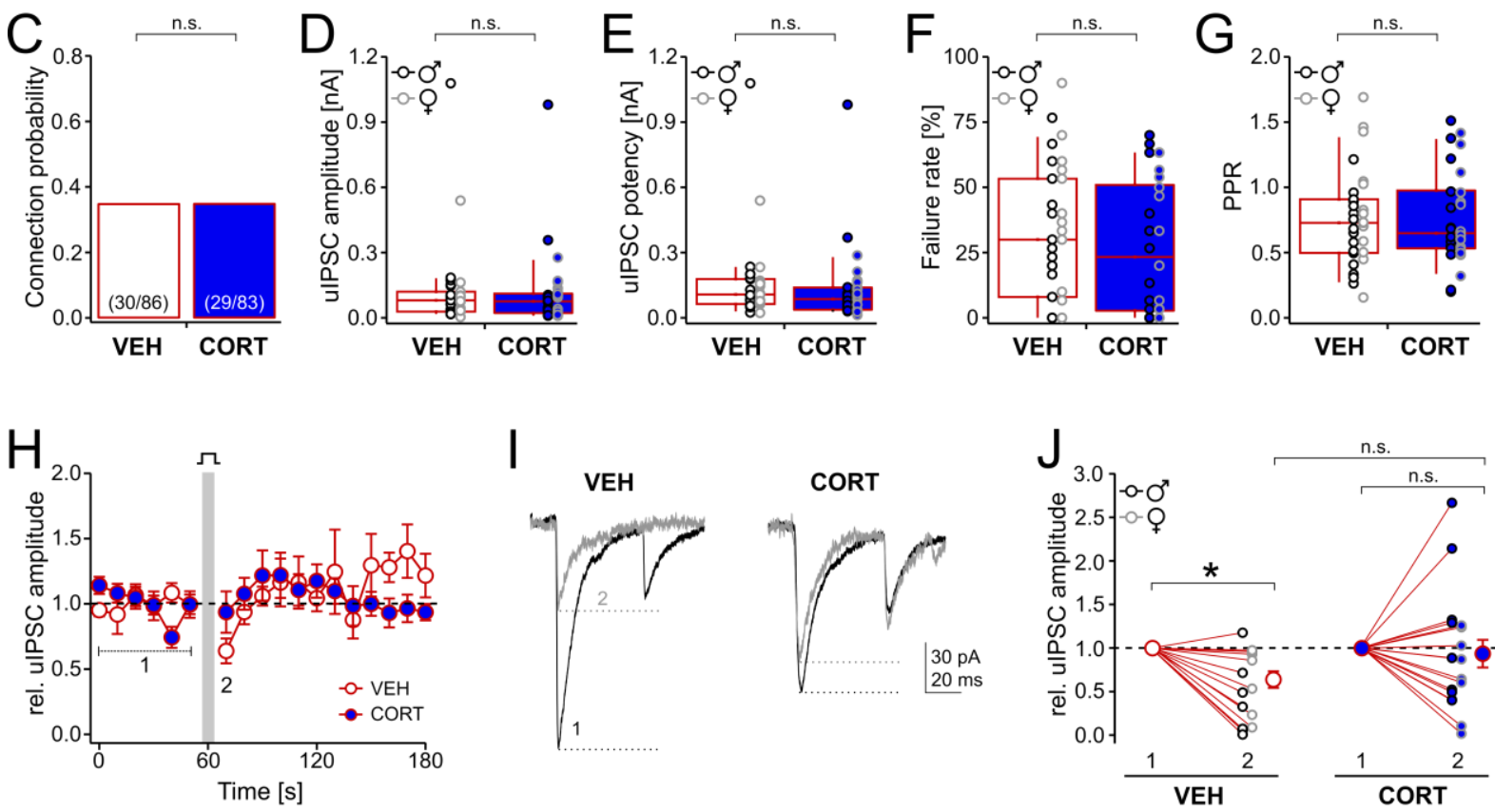

Figure 11: Chronic corticosterone treatment affects depolarization-induced suppression of synaptic inhibition but not basal synaptic transmission at GABAergic synapses made by CB1+ interneurons in prefrontal cortex. A) Illustration of experimental protocol. Mice received CORT $(0.1 \mathrm{mg} / \mathrm{ml}$ through the drinking water) for at least 20 days from P30 throughout adolescence, followed by pairwise CB1-to-PN recordings in mPFC L2/3 between P50-70 under continued drug treatment. VEH-treated controls received 1\% EtOH. Pairwise recordings were made in the presence of the glutamatergic AMPA receptor blocker NBQX (10 $\mu \mathrm{M})$. B) Example traces of synaptically connected CB1-to-PN pairs from a VEH- and CORT-treated mouse. C) Bar graph showing the connection probability of CB1+ INs onto PNs for VEH- and CORT-treated mice. The number of connected pairs / all painwise recordings is given in the parentheses. D-G) Summary graphs depicting (D) the uIPSC amplitude, (E) the uIPSC potency, (F) the failure rate, and (G) the PPR for connected CB1-to-PN pairs obtained from VEH (white) and CORT-treated mice (blue). Data are presented as box plots. Whiskers represent the $10^{\text {th }}$ and $90^{\text {th }}$ percentile. H) Summary graph depicting the time course of DSI in connected CB1-to-PN pairs obtained from VEH- and CORTtreated mice. Postsynaptic PN depolarization was performed after a $60 \mathrm{~s}$ baseline period (indicated by the gray bar). Shown is the baseline-normalized, relative uIPSC amplitude averaged over two consecutive sweeps, collapsed across sexes. I) Example traces of average uIPSCs recorded in PNs during the $60 \mathrm{~s}$ baseline period (1, black) and the $10 \mathrm{~s}$ period immediately after PN depolarization (2, gray) for a CB1-to-PN pair obtained from a VEHand CORT-treated mouse. J) Change in the relative uIPSC amplitude from the $60 \mathrm{~s}$ baseline period (1) to the $10 \mathrm{~s}$ period following PN depolarization (2). Dots in panels D-G) and J) represent individual data points, with males highlighted in black and females highlighted in gray. Panels $D-G): V E H: n / m=15 / 7$ for males, $n / m=15 / 6$ for females; CORT: $n / m=13 / 7$ for males, $n / m=16 / 7$ for females. Panels $H, J): V E H: ~ n / m=7 / 4$ for males, $n / m=7 / 5$ for females; CORT: $n / m=9 / 7$ for males, $n / m=8 / 5$ for females. Data were analyzed with Fisher's exact test, twoway art-ANOVAs, two-way ANOVAs, and one-tailed one-sample $t$-tests. ${ }^{*} p<0.05$. 
Similar to synapses made by PV + INs, GABAergic synapses made by CB1+ INs in mPFC were largely intact following chronic CORT treatment. The connection probability of CB1+ INs was highly comparable between CORT-treated mice (34.9\% [29/83 recorded pairs]) and VEH controls (34.9\% [30/86 recorded pairs]; $X^{2}=0.00, p>0.999$; Fisher's exact test; Figure 11C), indicating that the number of GABAergic synapses made by $\mathrm{CB} 1+$ INs remained unaltered by CORT exposure. Chronic CORT treatment during adolescence also did not affect the basal synaptic properties of these synapses, irrespective of sex (Figure 11B, D-G). There were no changes in the uIPSC amplitude (drug: $F_{(1,55)}=0.58, p=0.448$; sex: $F_{(1,55)}=0.06, p=0.808$; drug $\mathrm{x}$ sex: $F_{(1,55)}=0.04, p=0.837$; two-way art-ANOVA; $\mathrm{n} / \mathrm{m}=13-16$ connected pairs from 6-7 mice per drug treatment / sex; Figure 11D) and uIPSC potency (drug: $F_{(1,55)}=2.70, p=0.106$; sex: $F_{(1,55)}=0.40, p=0.530$; drug $x$ sex: $F_{(1,55)}=0.21, p=0.645$; two-way art-ANOVA; Figure 11E). Moreover, no changes were seen in the failure rate (drug: $F_{(1,55)}=0.68, p=0.413$; sex: $F_{(1,55)}=0.31, p=0.580$; drug $x$ sex: $F_{(1,55)}=0.00, p=0.948$; two-way art-ANOVA; Figure $11 \mathrm{~F}$ ) and PPR (drug: $F_{(1,55)}=0.05, p=0.823$; sex: $F_{(1,55)}=1.12, p=0.294$; drug $x$ sex: $F_{(1,55)}=0.03, p=0.863$; two-way art-ANOVA; Figure $11 \mathrm{G})$. Together, these results demonstrate that basal synaptic transmission at GABAergic synapses made by $\mathrm{CB} 1+$ INs in the mPFC is not affected by chronic CORT treatment during adolescence.

I further tested DSI in a subset of connected CB1-to-PN pairs to assess whether chronic CORT treatment affected retrograde eCB signaling at GABAergic synapses made by $\mathrm{CB} 1+\mathrm{INs}$. At CB1-to-PN pairs obtained from VEH controls, postsynaptic depolarization of PNs transiently decreased the relative uIPSC amplitude by $36.1 \%$ compared to baseline $\left(t_{(13)}=-3.42, p=0.002\right.$; one-tailed one-sample $t$-test collapsed across sexes; $\mathrm{n} / \mathrm{m}=14 / 9$; Figure $11 \mathrm{H}-\mathrm{J}$ ), indicating DSI. This effect was robust and consistently seen in the majority of tested pairs (Figure 11J). By contrast, such DSI was overall absent at synapses made by CB1+ INs in CORT-treated mice, as evidenced by the unchanged relative uIPSC amplitude following PN depolarization $\left(t_{(16)}=-\right.$ $0.38, p=0.355$ vs. baseline; one-tailed one-sample $t$-test collapsed across sexes; $n / m=17 / 12$; Figure $11 \mathrm{H}-\mathrm{J}$ ). Notably, the disrupting effect of CORT on DSI was highly variable, with some tested pairs showing a prominent decrease, others showing no change, and again others even showing an increase in the relative uIPSC amplitude following PN depolarization (Figure 11J). As such, DSI sensitivity overall did not differ between drug treatment groups and also no sexdependent effects were evident (drug: $F_{(1,27)}=1.89, p=0.181$; sex: $F_{(1,27)}=0.81, p=0.376$; drug $x$ sex: $F_{(1,27)}=1.10, p=0.303$; two-way ANOVA; $n / m=7-9$ pairs from 4-7 mice per drug treatment / sex; Figure 11G). The increased DSI variability at synapses made by CB1+ INs in CORTcompared to VEH-treated mice yet did not reach significance $\left(F_{(1,29)}=2.01, p=0.196\right.$, Levene test). Moreover, the strength of DSI at individual synapses should be interpreted with caution. However, in most cases, two DSI runs were averaged, including the pairs that showed an increase in the relative uIPSC amplitude rather than a decrease following PN depolarization. 
It is therefore unlikely that the high variance seen in CORT-treated mice is exclusively driven by a stochastic process. Collectively, these results suggest that chronic CORT treatment during adolescence disrupts phasic retrograde eCB signaling in a subset of GABAergic synapses made by CB1+ INs in the mPFC. 
4. Discussion 


\subsection{Summary of main findings}

Stress-induced dysfunctions in eCB signaling have been proposed to contribute to the etiology of psychiatric disorders, including schizophrenia, major depression, and anxiety disorders (McLaughlin et al., 2014; Lutz et al., 2015). On a cellular level, eCB signaling occurs in a retrograde manner and serves to modulate synaptic function through activation of presynaptic CB1 receptors (Castillo et al., 2012). These receptors are heavily expressed in certain neocortical IN types, so-called CB1+ INs, implicating them as potential cellular substrates of stress-induced disease states. However, their function and properties especially in the neocortex are poorly understood. I therefore studied the distribution, connectivity, and stress susceptibility of CB1+ INs in mouse neocortex. To this end, I compared them with fast-spiking $\mathrm{PV}+\mathrm{INs}$, a well-studied IN type with an established role in cortical network refinement (Freund and Katona, 2007; Tremblay et al., 2016).

This study has several main findings. First, I showed that CB1+ and PV+ INs represent anatomically and physiologically distinct IN types in neocortex, which considerably differ with respect to their intrinsic and synaptic properties. Secondly, I demonstrated that cortical hierarchy strongly determines how $\mathrm{CB} 1+$ and $\mathrm{PV}+\mathrm{INs}$ are recruited by different cortical regions to exert inhibitory control over excitatory networks. Specifically, I found that PV+ INs were considerably more abundant and formed more GABAergic synapses onto pyramidal neurons compared to $\mathrm{CB} 1+\mathrm{INs}$ in primary somatosensory cortex. By contrast, the abundance and inhibitory connectivity was largely balanced between PV+ and CB1+ INs in the prefrontal cortex, a higher-order associative cortical structure that has been implicated in the etiology of various psychiatric diseases (Arnsten, 2009; Popoli et al., 2011; Page and Coutellier, 2019). Interestingly, further experiments revealed that GABAergic synapses made by the two IN types in prefrontal cortex matured rather early in development. Moreover, these synapses were highly resistant to the effects of stress-related glucocorticoid signaling, using chronic CORT treatment during adolescence, the presumed critical period of prefrontal development (Spear, 2000; Caballero and Tseng, 2016). However, chronic CORT exposure during adolescence selectively impaired retrograde eCB-dependent synaptic plasticity at GABAergic synapses made by $\mathrm{CB} 1+$ INs. Together, these findings point to an important role of CB1+ INs in higherorder cortical structures such as the prefrontal cortex and suggest them as potential targets of stress-induced alterations in eCB signaling.

\section{2. $\quad \mathrm{CB} 1+$ and $\mathrm{PV}+$ interneurons represent anatomically and physiologically distinct cell types in neocortex}

Previous studies suggested that CB1 receptors mainly localize onto presynaptic terminals of CCK+ INs, while PV+ INs are almost completely devoid of these receptors (Katona et al., 1999; Marsicano and Lutz, 1999; Bodor et al., 2005; Hill et al., 2007). Consistently, using double reporter mice, I showed that $\mathrm{CB} 1+$ and $\mathrm{PV}+\mathrm{INs}$ represent anatomically non-overlapping, 
mutually exclusive cell types in neocortex (Figure 1,2). Patch-clamp recordings in the mPFC further revealed that the two IN types exhibit distinct intrinsic physiological properties. PV+ INs showed the typical fast-spiking behavior, while CB1+ INs were either regular-spiking or fastaccommodating (with occasional irregular firing). Interestingly, prefrontal CB1+ INs required less current to fire APs and were more depolarized at resting potential compared to PV+ INs (Figure 1). Although not systematically assessed, I observed similar properties for $\mathrm{CB} 1+$ and $\mathrm{PV}+\mathrm{INs}$ in $\mathrm{S} 1$. Of note, the properties of $\mathrm{CB} 1+\mathrm{INS}$ found in the present study are largely comparable with those that have been described for CCK+ INs in the hippocampus (e.g. Neu et al., 2007; Ali, 2007; Lee et al., 2010b) and more recently the mPFC (Nguyen et al., 2020). They thus resonate well with studies showing that CB1 receptors are preferentially expressed in CCK+ INs (Katona et al., 1999; Marsicano and Lutz, 1999; Bodor et al., 2005; Hill et al., 2007). Together, these data demonstrate that $C B 1+$ and $P V+I N s$ represent anatomically and physiologically distinct cell types in neocortex.

Remarkably, my data are in sharp contrast with our previous characterization of CB1+ INs in the NAc. In fact, we demonstrated that NAc CB1+ INs exhibit fast-spiking-like firing properties and also co-express PV in $\sim 40 \%$ of cases (Winters et al., 2012), indicating a substantial overlap between $\mathrm{CB} 1+$ and PV+ INs in subcortical areas. These results suggest that CB1+ INs can fully switch their physiological profile depending on target structure and thus point to a differential cell type-specific targeting of CB1 receptors in neocortical and subcortical regions. The mechanisms for this are not known, but potential candidates could be cell type-specific differences in the post-transcriptional regulation of $\mathrm{CB} 1$ receptor expression. Indeed, $\mathrm{CB} 1$ receptor mRNA is abundantly expressed in various other neuron types besides CCK+ INs, including SST+ and VIP+ INs (Hill et al., 2007). However, most of these transcripts are apparently not translated into functional protein (Bodor et al., 2005). Together, these findings point to the existence of different region- and cell type-specific post-transcriptional programs that govern the recruitment of $\mathrm{CB} 1$ receptors to specific neuron populations. Future studies should therefore assess the protein expression patterns of $\mathrm{CB} 1+\mathrm{INs}$ in different brain regions using immunostainings for various IN marker proteins in order to determine their exact molecular identity.

\subsubsection{CB1+ interneurons display a high intrinsic excitability but receive only weak excitatory glutamatergic inputs compared to PV+ interneurons}

CB1+ INs displayed a higher intrinsic excitability compared to PV+ INs in the present study. Specifically, they showed steeper I-V curves, required less current to fire APs, and displayed substantially more depolarized resting potentials compared to PV+ INs (Figure 1), suggesting that they can be readily recruited during cortical network activity. Inconsistent with this notion, they yet received considerably fewer and weaker glutamatergic synaptic inputs compared to $\mathrm{PV}+\mathrm{INs}$, as evidenced by the lower frequency and amplitude of AMPA receptor-mediated 
mEPSCs (Figure 7). These data are in line with findings demonstrating a rather weak excitatory glutamatergic drive onto CB1+ INs in the hippocampus (Glickfeld and Scanziani, 2006). What inputs then drive these neurons to fire?

Several lines of evidence suggest that CCK + INs in neocortex and hippocampus are under strong regulatory control by different neuromodulators, such as acetylcholine and serotonin (Freund, 2003; Freund and Katona, 2007). For instance, CCK+ INs express different nicotinic and muscarinic acetylcholine receptors (Porter et al., 1999; Cea-del Rio et al., 2010). Activation of these receptors can strongly excite these cells and thereby increase their functional output (Porter et al., 1999; Karson et al., 2009; Cea-del Rio et al., 2010). Indeed, the cholinergic agonist carbachol potently increases the frequency of spontaneous IPSCs (sIPSCs) in pyramidal neurons, presumably through activation of CCK+ INs (Kawaguchi, 1997; Martin and Alger, 1999). These carbachol-induced SIPSCs are highly prone to undergo CB1 receptordependent DSI (Martin and Alger, 1999; Wilson and Nicoll, 2001; Trettel et al., 2004; Bodor et al., 2005; Yoshino et al., 2011), indicating that a similar cholinergic control of excitation is present at $\mathrm{CB} 1+\mathrm{INs}$. Moreover, the activity of $\mathrm{CB} 1+\mathrm{INs}$ is possibly under serotonergic regulation. For instance, many CCK + INs are strongly excited by serotonin, presumably through activation of serotonergic 5-HT3a receptors (Férézou et al., 2002), which are frequently co-expressed together with CB1 receptors (Morales and Bäckman, 2002; Morales et al., 2004). Future studies should therefore examine the cholinergic and serotonergic excitability of $\mathrm{CB} 1+$ INs. Furthermore, it would be interesting to assess whether such modulatory activity acts in concert with glutamatergic activity, e.g. by gating the recruitment of CB1+ INs through specific feedforward excitatory glutamatergic inputs. This could be tested e.g. by determining the change in EPSP-spike coupling in CB1+ INs following bath application of different neuromodulators.

It should be noted that both cholinergic and serotonergic activity have been associated with different motivational and emotional states (Freund, 2003; Freund and Katona, 2007). Hence, such experiments would possibly also shed light on the role of $\mathrm{CB} 1+$ INs in different affective states, with possible implications for the study of mood disorders, such as depression and anxiety disorders.

\subsubsection{CB1+ interneurons form less reliable and precise GABAergic synapses compared to PV+ interneurons}

A central observation of the present study was that CB1+ INs often formed highly unreliable GABAergic synapses, which were characterized by a high incidence of transmission failures (Figure 5, 7, 11). In sharp contrast, PV+ INs almost exclusively formed strong and highly reliable synapses, which were usually characterized by a complete absence of transmission failures, as described previously (Hefft and Jonas, 2005; Galarreta et al., 2008). This pattern was evident throughout the neocortex and frequently associated with corresponding 
differences in the PPR, indicating a lower Prat GABAergic synapses made by CB1+ compared to $\mathrm{PV}+\mathrm{INs}$. Similar properties have been described for inhibitory synapses made by electrophysiologically identified irregular-firing CB1+ INs in somatosensory cortex (Galarreta et al., 2008) and CCK/CB1 + INs in hippocampus (Losonczy et al., 2004; Hefft and Jonas, 2005; Neu et al., 2007; Karson et al., 2009; Lee et al., 2010b; 2015).

The lower fidelity of synaptic transmission at synapses made by CB1+ INs could be caused by less precise presynaptic $\mathrm{Ca}^{2+}$ signaling. Indeed, CCK/CB1+ and PV+ INs in hippocampus express different VGCCs on their presynaptic terminals, which are sensitive to different neurotoxins and endow them with different exocytotic release properties. PV+ INs express $\mathrm{P} / \mathrm{Q}$-type $\mathrm{Ca}^{2+}$ channels, which are sensitive to $\omega$-agatoxin. They are typically located close to the presynaptic active zone and thus associated with a tight coupling between $\mathrm{Ca}^{2+}$ influx and the $\mathrm{Ca}^{2+}$ sensor, thereby allowing PV+ INs to exert fast, synchronous synaptic inhibition with a high $\operatorname{Pr}$ (Hefft and Jonas, 2005). By contrast, CCK/CB1+ INs express $\mathrm{N}$-type $\mathrm{Ca}^{2+}$ channels, which are sensitive to $\omega$-conotoxin. They are usually located more distal to the presynaptic active zone and thus associated with a loose coupling between $\mathrm{Ca}^{2+}$ influx and the $\mathrm{Ca}^{2+}$ sensor, resulting in a low Prand largely asynchronous GABA release at these synapses (Wilson et al., 2001; Hefft and Jonas, 2005; Földy et al., 2006; Karson et al., 2009). Consequently, the high failure rates at synapses made by neocortical $\mathrm{CB} 1+\mathrm{INs}$ seen in the present study could be due to presynaptic N-type VGCCs conferring a looser coupling between presynaptic $\mathrm{Ca}^{2+}$ signaling and the $\mathrm{Ca}^{2+}$ sensor. Consistently, GABAergic responses occurred at longer and more variable synaptic delays at these synapses (Figure 5), which is in line with other studies (Hefft and Jonas, 2005; Neu et al., 2007; Galarreta et al., 2008).

Additionally, the high incidence of transmission failures at synapses made by CB1+ INs could be driven by persistent activity of CB1 receptors. Such persistent receptor activity has been demonstrated at GABAergic synapses made by CCK/CB1+ INs in hippocampus (Neu et al., 2007; Lee et al., 2010b; 2015). Indeed, some of these synapses are under such strong control through persistently active CB1 receptors that they are almost completely muted (Losonczy et al., 2004). Pharmacological blockade of CB1 receptors with AM251 can release this brake and convert these synapses into highly reliable ones (Losonczy et al., 2004; Neu et al., 2007; Lee et al., 2010b; 2015). AM251 acts as a CB1 receptor antagonist / inverse agonist and is thus able to block constitutive receptor activity in addition to eCB-mediated activation. Indeed, several lines of evidence indicate that CB1 receptors can be constitutively active and thus could potentially suppress GABA release even in the absence of eCB signaling. For instance, Lee et al. (2015) demonstrated that the failure rates of GABAergic responses at synaptically connected pairs of $\mathrm{CB} 1+\mathrm{INs}$ and $\mathrm{CA} 1$ pyramidal neurons were reduced only in the presence of AM251 but not the neutral CB1 receptor antagonist NESS0327, which selectively blocks the receptor-binding site without modulating constitutive receptor activity. However, NESS0327 
effectively blocked DSI and thus phasic eCB signaling (Lee et al., 2015). Although an additional role of tonic eCB signaling cannot be ruled out (Neu et al., 2007; Lee et al., 2010b; 2015), these findings suggest that the high rates of transmission failures are strongly driven by constitutive CB1 receptor activity. Consistently, I found that the basal synaptic properties, including the failure rates, at synapses made by prefrontal $\mathrm{CB} 1+$ INs were completely unaffected in the presence of NESS0327 (Supplementary Figure 1), although it successfully blocked DSI (Figure 4). It would be therefore interesting to record from synaptically connected pairs in the presence of AM251 to assess whether a similar regulation through constitutively active $\mathrm{CB} 1$ receptors operates in neocortex.

Notably, the failure rates were not uniform across individual GABAergic synapses. On the contrary, I observed substantial variability, ranging from high-fidelity synapses with low failure rates to extremely unreliable, almost silent synapses with only few successful responses (Figure 5). It is possible that the variability in failure rates is the expression of a certain form of experience-dependent synaptic plasticity induced at individual GABAergic synapses. Indeed, synapses made by $\mathrm{CB} 1 / \mathrm{CCK}+$ INs have a high propensity to undergo different forms of plasticity. The most prominent example is DSI, a short-term form of eCB-dependent synaptic plasticity, which was also observed in the present study (e.g. Wilson et al., 2001; Wilson and Nicoll, 2001; Földy et al., 2006; Lee et al., 2010b; Figure 4). Additionally, these synapses have the ability to undergo so-called LTDi, a long-term form of CB1 receptor-dependent depression of presynaptic GABA release that is triggered by group I mGlu receptor-mediated retrograde eCB signaling following repetitive activation of excitatory glutamatergic synapses (Chevaleyre and Castillo, 2003; 2004; Chevaleyre et al., 2006). Once induced, LTDi becomes independent of $\mathrm{CB} 1$ receptor activation and is expressed via a reduction in presynaptic cAMP/PKA signaling (Chevaleyre and Castillo, 2003; Chevaleyre et al., 2007). Interestingly, previous studies suggested that such eCB-dependent long-term plasticity can be induced by experience in vivo (Jiang et al., 2010a; Huang et al., 2010) and is expressed at individual synapses in the form of reduced uIPSC amplitudes and an increased incidence of transmission failures (Heifets et al., 2008). These findings raise the possibility that the high failure rates at some GABAergic synapses made by CB1 + INs might reflect the expression of an experience-dependent LTDi in vivo. This could be tested by assessing whether the induction of eCB-dependent LTDi is occluded at unreliable synapses and whether unreliable synapses can be converted into reliable ones by reverting eCB-dependent LTDi, e.g. by enhancing presynaptic cAMP/PKA signaling. Importantly, all these mechanisms are not mutually exclusive, but could operate in parallel to determine the fidelity of synaptic transmission at individual GABAergic synapses made by CB1+ INs. 


\subsection{Cortical hierarchy shapes inhibitory network motifs formed by CB1+ and PV+ interneurons}

It is widely believed that PV+ INs represent the major perisomatic IN type in neocortex (Rudy et al., 2011; Fino et al., 2013; Tremblay et al., 2016). Indeed, PV+ INs show a dense inhibitory connectivity onto neocortical pyramidal neurons and have been estimated to make up $\sim 40 \%$ of the total IN population (Lee et al., 2010a; Xu et al., 2010; Packer and Yuste, 2011; Pfeffer et al., 2013; Whissell et al., 2015). However, these findings are largely restricted to primary sensory systems. By contrast, information about higher-order associative structures is often lacking and other IN types are frequently neglected. In the present study, I sought to bridge this gap of knowledge by assessing the distribution and inhibitory connectivity of PV+ and $\mathrm{CB} 1+\mathrm{INs}$ in S1 and mPFC as representative cortical regions for basal sensory and higherorder cognitive functions.

I found that PV+ INs were considerably more abundant and made substantially more GABAergic synapses onto pyramidal neurons compared to CB1+ INs in S1. By contrast, both the abundance and inhibitory connectivity were largely balanced between PV+ and CB1+ INs in mPFC. These data challenge the traditional view of a dominant function of PV+ INs in cortical network refinement and reveal that cell type-specific inhibitory circuit motifs are strongly shaped by cortical hierarchy.

\subsubsection{Inhibitory control is dominated by $\mathrm{PV}+$ interneurons in primary somatosensory cortex}

Consistent with previous studies (Xu et al., 2010; Lee et al., 2010a; Whissell et al., 2015), I found that PV+ INs heavily populated most layers of S1, suggesting that they represent an abundant IN type in this region. By contrast, CB1+ INs were generally sparse throughout the different S1 layers and almost absent in S1 output layer L5/6 (Figure 2). My findings are in line with the lower density of CCK+ compared to PV+ INs in primary sensory and motor systems (Whissell et al., 2015). These results suggest that CB1+ INs only represent a secondary IN type compared to PV+ INs in these cortical regions.

Remarkably, the differences in the abundance of the two IN types were closely mirrored by corresponding differences in their functional inhibitory connectivity as obtained through pairwise patch-clamp recordings (Figure 5). Specifically, I found that PV+ INs formed GABAergic synapses onto pyramidal neurons in S1 L2/3 with a high connection probability of $\sim 70 \%$, which supports previous findings in $\mathrm{S} 1$ and other primary sensory areas (Holmgren et al., 2003; Oswald et al., 2009; Packer and Yuste, 2011; Pfeffer et al., 2013; Xue et al., 2014). By contrast, CB1 + INs showed a substantially lower connection probability of only $\sim 30 \%$. My results are highly consistent with a study by Galarreta et al. (2008), who found a significantly lower connection probability onto pyramidal neurons in electrophysiologically identified irregular-firing, presumably $\mathrm{CB} 1+\mathrm{INs}$ compared to fast-spiking, presumably PV+ INs in 
somatosensory cortex. These irregular-firing INs displayed eCB-dependent DSI at their GABAergic synapses onto pyramidal neurons and showed CB1 receptor immunoreactivity in $\sim 78 \%$ of cases, as opposed to fast-spiking INs (Galarreta et al., 2004; 2008). My findings also resonate well with immunocytochemical evidence that pyramidal cell somata in somatosensory cortex are more densely innervated by PV- compared to CB1-immunoreactive (-ir) axon terminals (Bodor et al., 2005). Indeed, Bodor et al. (2005) estimated that the proportion of CB1relative to all CB1- and PV-ir terminals onto individual pyramidal cell somata usually ranges between $30-40 \%$. These authors further observed pronounced laminar differences by showing that many pyramidal neurons in output layer 5B were nearly devoid of CB1-ir perisomatargeting terminals, which is in line with the layer-specific distribution of CB1+ INs observed in the present study (Figure 2). Consistently, using patch-clamp recordings, Bodor et al. (2005) further showed that inhibitory inputs on a large fraction of L5B pyramidal neurons display little to no CB1 receptor-dependent synaptic modulation. Fortin and Levine (2007) acquired similar findings. Collectively, these results suggest that perisomatic inhibitory control and cortical network refinement in primary sensory areas are largely governed by the activity of PV+ INs. Conversely, CB1+ INs only seem to serve a secondary function in perisomatic inhibition in primary sensory regions.

\subsubsection{Inhibitory control is balanced between $\mathrm{PV}+$ and $\mathrm{CB} 1+$ interneurons in prefrontal cortex}

A completely different picture emerged at the level of the mPFC, an important higher-order associative cortical structure implicated in executive control and mood regulation (Miller, 2000; Arnsten, 2009). Specifically, I found that the abundance of PV+ and CB1+ INs was largely balanced in the mPFC (Figure 2). These results are in agreement with the findings by Whissell et al. (2015), who found that CCK+ INs were only outnumbered by PV+ INs in sensory and motor cortices, but not the mPFC. Indeed, these authors showed that CCK+ INs even represented a larger fraction of the total IN population compared to PV+ INs in mPFC (Whissell et al., 2015; Nguyen et al., 2020). Interestingly, this pattern was maintained throughout the different prefrontal cortical layers (Nguyen et al., 2020). In contrast, I found that the abundance was largely comparable between $\mathrm{CB} 1+$ and $\mathrm{PV}+\mathrm{INs}$ in the mPFC, especially in L2/3. Moreover, I observed prominent laminar differences, as evidenced by a lower density of CB1+ compared to PV+ INs in mPFC layers L5/6 (Figure 2). These differences might be due to several reasons. Firstly, there is only partial overlap between CB1+ and CCK+ INs (Marsicano and Lutz, 1999; Bodor et al., 2005; Hill et al., 2007). Secondly, CCK-Cre driver lines, as employed by Whissell et al. (2015) and Nguyen et al. (2020), strongly lack specificity since CCK is also abundantly expressed in glutamatergic pyramidal neurons (Hill et al., 2007). The authors partially overcame this problem by using an intersectional, dual Flipase/Cre recombinase approach to selectively label GABAergic neurons. However, Nguyen et al. (2020) 
observed that their strategy still non-specifically co-labeled a substantial subset of PV+ INs. Indeed, they found that a $\sim 20 \%$ fraction of putative prefrontal CCK+ GABAergic neurons coexpressed PV and displayed fast-spiking properties, thus rather representing a subpopulation of PV+ INs, which likely biased their results. By contrast, the labeling approach employed here was highly specific, with zero overlap between CB1+ and PV+ INs. Despite these differences, these data provide strong anatomical support for the idea that cell type-specific inhibitory circuit motifs are governed by cortical hierarchy.

In support of this notion, using pairwise patch-clamp recordings, I further found that the relative inhibitory connectivity of the two IN types completely changed between S1 and mPFC. While $\mathrm{PV}+\mathrm{INs}$ formed considerably more GABAergic synapses onto pyramidal neurons compared to $\mathrm{CB} 1+$ INs in S1, the inhibitory connection probability was largely balanced between the two IN types in mPFC, generally ranging between 30-40\%. Importantly, this pattern was highly robust and could be replicated in three independent data sets, at two different developmental time points, and in mice chronically treated with CORT (Figure 5, 8, 10, 11). Consistently, a $30-40 \%$ connection probability has been observed before for PV+ INs in mPFC output layers L5/6 (Yang et al., 2014). Of note, this balanced mode of cell type-specific prefrontal inhibition was primarily driven by a regional decrease in the abundance and inhibitory connectivity of $\mathrm{PV}+\mathrm{INs}$ from S1 to mPFC. By contrast, the density and inhibitory connectivity of CB1+ INs were rather stable between the two cortical regions (Figure 2, 5). However, the decrease in $\mathrm{PV}+\mathrm{IN}$-mediated inhibitory control likely also recalibrates the functional significance of CB1+ INs, by conferring a higher relative synaptic weight to these neurons. Collectively, my data suggest that a PV+ IN-dominated circuit motif in sensory systems is replaced by a more symmetrical motif in the mPFC, where inhibitory control is largely balanced between PV+ and CB1+ INs. These findings are in line with studies showing strong cannabinoid modulation of prefrontal GABA transmission and circuit function (Chiu et al., 2010; Kucewicz et al., 2011; Cass et al., 2014; Szkudlarek et al., 2019) and point to an increased importance of CB1+ INs in regulating cortical circuit activity with relevance to higher-order processes, such as cognitive control and mood regulation.

\subsubsection{CB1+ interneurons form distinct subtypes of GABAergic synapses in primary somatosensory but not prefrontal cortex}

I found that a fraction of CB1+ INs formed a region-specific subtype of atypical GABAergic synapses onto pyramidal neurons, which were characterized by unusually slow signaling kinetics. These so-called slow type-II synapses could be easily distinguished from the majority of typical, fast type-I synapses, were only formed by $\mathrm{CB} 1+$ but not PV+ INs, and were exclusively seen in S1, but not mPFC (Figure 6; Supplementary Figure S5, S6). Transmission at slow type-II synapses was likely mediated by ionotropic $\mathrm{GABA}_{\mathrm{A}}$ receptors, as postsynaptic GABA responses were recorded in the presence of $\mathrm{CsCl}$ and $\mathrm{QX}-314$ in the internal solution 
to prevent $\mathrm{GABA}_{\mathrm{B}}$ receptor-mediated potassium signaling and completely depressed by PTX (1 connected pair; data not shown). Similar distinctions of fast and slow GABAergic synapses have been made in hippocampus (Pearce, 1993; Banks et al., 2000). These results demonstrate that $\mathrm{CB} 1+\mathrm{INs}$ form region-specific subtypes of fast type-I and slow type-II GABAergic synapses in neocortex.

Only speculations can be made about their identity. However, the different signaling kinetics point to differences in the subcellular targeting of synapses and/or subunit composition of postsynaptic $\mathrm{GABA}_{\mathrm{A}}$ receptors. Evidence suggests that $\mathrm{CCK} / \mathrm{CB} 1+\mathrm{INs}$ usually form $\alpha 2$ subunit-containing perisomatic GABAergic synapses onto postsynaptic pyramidal neurons (Thomson et al., 2000; Nyíri et al., 2001). These $\alpha 2$-containing perisomatic synapses made by CCK/CB1+ INs present rather fast signaling kinetics (Hefft and Jonas, 2005; Földy et al., 2006; Neu et al., 2007; Galarreta et al., 2008; Ali and Thomson, 2008; Lee et al., 2010b), corresponding to the signaling properties of fast type-I synapses as the predominant synapse type made by $\mathrm{CB} 1+$ INs in the present study. By contrast, slow type-II synapses might preferentially express a5 subunit-containing GABA $_{A}$ receptors, which have previously been associated with slow signaling properties at both hippocampal and neocortical GABAergic synapses (Ali and Thomson, 2008; Schulz et al., 2018). Interestingly, these receptors seem to be preferentially localized at synapses made by dendrite-targeting, nitric oxide synthaseexpressing (NOS+) and SST+ INs (Ali and Thomson, 2008; Schulz et al., 2018), pointing to the existence of a subpopulation of NOS+ and/or SST+ CB1+ INs in S1 that form dendritic, a5-containing GABAergic synapses. Notably, a5-containing GABAergic synapses exhibit strongly outwardly-rectifying channel properties (Schulz et al., 2018). Thus, the presence of a5-containing $\mathrm{GABA}_{\mathrm{A}}$ receptors at slow type-II synapses could be tested by examining rectification indices at synaptically connected pairs of $\mathrm{CB} 1+\mathrm{INs}$ and pyramidal neurons. These recordings could be further combined with single-cell RNA sequencing to determine the molecular identity of CB1+ INs that form slow type-II synapses.

Interestingly, slow type-II synapses also considerably differed from fast type-I synapses with respect to their presynaptic release properties. As outlined in previous sections, CB1+ INs often form highly unreliable GABAergic synapses characterized by high failure rates and more variable synaptic delays, which has been linked to the expression of presynaptic N-type VGCCs and persistent CB1 receptor activity (Losonczy et al., 2004; Hefft and Jonas, 2005; Neu et al., 2007; Lee et al., 2010b; 2015). Remarkably, the present results suggest that these properties might be exclusive to fast type-I synapses. Indeed, I found that unreliable synapses were only present among fast type-I synapses. By contrast, slow type-II synapses displayed a complete absence of transmission failures and exhibited considerably shorter synaptic delays (Figure 6, Supplementary Figure S5). In addition to possible differences in postsynaptic GABA receptor subunit composition, slow type-II synapses may thus potentially also express different 
types of presynaptic VGCCs and/or only phasically active CB1 receptors. Indeed, Wilson et al. (2001) identified a subtype of slow GABAergic synapses in hippocampus with similar kinetic properties as the slow type-II synapses observed here, which seemed to express a combination of N-type and P/Q-type VGCCs. Interestingly, these slow synapses were insensitive to DSI (Wilson et al., 2001). It would therefore be also important to characterize the presynaptic properties of slow type-II synapses, such as their eCB sensitivity. Taken together, these data suggest that slow type-II synapses represent a unique subpopulation of GABAergic synapses exclusively formed by $\mathrm{CB} 1+\mathrm{INs}$ in $\mathrm{S} 1$ but not mPFC. These findings thus add further support to the notion that cortical hierarchy shapes the properties of inhibitory networks made by different IN types.

\subsection{Stress-related glucocorticoid signaling during adolescence induces selective alterations in endocannabinoid signaling at synapses made by CB1+ interneurons in prefrontal cortex}

The prefrontal cortex is highly susceptible to the disrupting effects of chronic stress. This stress vulnerability is particularly pronounced during adolescence, a transitional phase between the juvenile stage and adulthood that is considered the critical developmental period for prefrontal network refinement (Spear, 2000; Gee and Casey, 2015; Caballero and Tseng, 2016). Interestingly, stress alters eCB signaling in prefrontal cortex and aberrant prefrontal eCB signaling has been implicated in various stress-related disorders, including schizophrenia, major depression, and anxiety disorders. These findings suggest prefrontal CB1+ INs as an important substrate of stress-induced dysfunction (McLaughlin et al., 2014). I therefore assessed the developmental trajectories and stress susceptibility of inhibitory networks made by $\mathrm{CB} 1+$ compared to PV+ INs in the mPFC during adolescence.

I found that the inhibitory output connectivity and excitatory input connectivity of prefrontal $\mathrm{PV}+$ and CB1+ INs was established early in development before the onset of the adolescent phase. Remarkably, I found that stress-related glucocorticoid signaling using chronic CORT treatment during adolescence did not affect basal synaptic transmission at GABAergic synapses made by both IN types, but induced a selective impairment in eCB-dependent retrograde control of synaptic inhibition through prefrontal $\mathrm{CB} 1+\mathrm{INs}$. Collectively, these results indicate that prefrontal $\mathrm{CB} 1+\mathrm{INs}$ may act as an important cellular substrate linking changes in eCB signaling with stress-induced disease states.

\subsubsection{Inhibitory networks made by prefrontal PV+ and CB1+ interneurons mature early during development}

In sensory systems, inhibitory circuits undergo pronounced developmental changes during socalled critical periods in order to reach their functional maturity (Hensch, 2005). I therefore sought to determine possible developmental changes in GABAergic synaptic transmission 
through $\mathrm{CB} 1+$ and $\mathrm{PV}+\mathrm{INs}$ in the mPFC during adolescence, the presumed critical period of prefrontal cortical development (Spear, 2000; Gee and Casey, 2015; Caballero and Tseng, 2016). Surprisingly, I found that the inhibitory connectivity of $P V+$ and $C B 1+I N s$ in the mPFC was largely matured before the onset of the adolescent phase. Indeed, GABAergic synapses made by the two IN types onto prefrontal pyramidal neurons seemed to be fully established and exhibited adult-like properties already during the juvenile stage (Figure 7). Similarly, I found only moderate developmental changes in the frequency and amplitude of AMPA receptor-mediated mEPSCs in the two IN types, suggesting that also their excitatory glutamatergic input synapses were mostly matured before adolescence (Figure 8). However, I observed some decrease in the mEPSC frequency in prefrontal PV+ INs during adolescence, consistent with synapse pruning. Together, these findings suggest that both the inhibitory output and excitatory input connectivity of the two IN types are largely established in mPFC before the onset of the adolescent phase.

The observed lack of developmental changes in prefrontal PV+ INs seemingly contradicts with studies on the maturation of these neurons in primary sensory systems, where they are thought to govern the onset and duration of critical period plasticity, such as in the primary visual cortex V1 (Hensch et al., 1998; Huang et al., 1999; Jiang et al., 2010b; Kuhlman et al., 2013; for review see: Hensch, 2005; Le Magueresse and Monyer, 2013). However, it has been suggested that the formation of glutamatergic inputs onto PV+ INs drives PV expression, which ultimately promotes the maturation of their GABAergic output synapses (Caballero and Tseng, 2016). Since I identified neurons based on their PV expression using a PV-Cre driver line, it is therefore possible that I generally recorded only from mature PV+ INs. Indeed, I noticed a lower number of PV+ INs during the P20-30 recordings, indicating that not all PV+ INs in the mPFC expressed PV yet during the juvenile stage, which is consistent with previous findings (Caballero et al., 2014). It would be therefore interesting to determine the developmental trajectories of $\mathrm{PV}$ expression in prefrontal $\mathrm{PV}+\mathrm{INs}$ in more detail by assessing their cell density across postnatal development. Moreover, possible maturational changes in the excitatory input and inhibitory output connectivity of prefrontal PV+ INs could be investigated in G42 reporter mice, which label fast-spiking PV+ INs independently of PV expression and hence independently of their maturational state (Chattopadhyaya et al., 2004). Of note, previous studies in G42 reporter mice suggested that GABAergic synapses between PV+ INs and pyramidal neurons in both V1 and mPFC are mostly established by $\sim \mathrm{P} 15$ and thus before the onset of the critical period (Pangratz-Fuehrer and Hestrin, 2011; Yang et al., 2014). However, these findings were obtained in output layers $L 5 / 6$, as opposed to the input layers $L 2 / 3$ examined in the present investigation.

Moreover, the observed lack of developmental changes at GABAergic synapses made by prefrontal CB1+ INs seems contradictory to studies on eCB-dependent LTDi in visual cortex 
during development. Jiang et al. (2010a) showed that the maturation of the V1 cortical GABAergic circuitry is regulated by a progressive, experience-dependent induction of eCBdependent LTDi at GABAergic synapses during the critical period. This putative in vivo LTDi occurs between the $3^{\text {rd }}$ and $4^{\text {th }}$ postnatal week, seems to be mediated by an experience-driven release of eCBs, and is associated with a developmental reduction in the presynaptic Prat $\mathrm{V} 1$ GABAergic inputs (Huang et al., 2010; Jiang et al., 2010a). It has been further shown that eCBdependent LTDi is expressed at individual GABAergic synapses in the form of reduced uIPSC amplitudes and increased failure rates (Heifets et al., 2008). By contrast, in the present study, I did not observe a developmental decrease in the strength or reliability of synapses made by prefrontal CB1+ INs during the critical period of adolescence (Figure 7). However, a lack of changes in these basal synaptic properties does not necessarily imply an absence of a developmental form of eCB-dependent LTDi in the mPFC as compared to V1. For instance, several independent development changes could occur in parallel, which could mask or cancel out possible effects of LTDi at GABAergic synapses, such as a parallel increase in presynaptic release sites and/or postsynaptic $\mathrm{GABA}_{\mathrm{A}}$ receptor density (Jiang et al., 2010b). Indeed, the induction of eCB-dependent LTDi during V1 maturation is accompanied by a paradoxical enhancement of cortical inhibition rather than depression, whereas the ability for LTDi in immature V1 neurons is associated with reduced cortical inhibition (Jiang et al., 2010b; Huang et al., 2010). Moreover, it has been shown that eCB-dependent LTDi in V1 is not necessarily permanent, but can be reversed through experience, indicating that GABAergic synapses can rejuvenate and regain their ability to undergo LTDi (Huang et al., 2010). Such a process might be particularly important in the MPFC, which possibly has to maintain a high degree of juvenilelike plasticity in order to perform higher-order cognitive functions.

The developmental induction of LTDi in V1 was unexpectedly reported to occur at GABAergic synapses of PV+ INs (Jiang et al., 2010a). It would be therefore highly interesting to assess possible developmental changes in the sensitivity of GABAergic synapses of both prefrontal $\mathrm{PV}+$ and $\mathrm{CB} 1+\mathrm{INs}$ to undergo eCB-dependent LTDi.

\subsubsection{Chronic corticosterone treatment during adolescence does not affect basal synaptic transmission at GABAergic synapses made by prefrontal PV+ and CB1+ interneurons}

Stress-induced prefrontal circuit dysfunction has been assumed to be partly mediated by a malfunctioning in the local GABAergic circuitry. However, reported findings are often conflicting and the role of specific cell types has remained elusive (Ma et al., 2016; McKlveen et al., 2016; Shepard et al., 2016; Czéh et al., 2018; Ghosal et al., 2020; for review: Page and Coutellier, 2019). I therefore assessed possible cell type-specific changes in synaptic inhibition mediated by $\mathrm{PV}+$ and $\mathrm{CB} 1+\mathrm{INs}$ in mPFC following chronic CORT treatment during adolescence as a model of glucocorticoid-mediated stress (Moda-Sava et al., 2019). 
I found that both the number and strength of GABAergic synapses made by prefrontal PV+ and $\mathrm{CB} 1+$ INs onto pyramidal neurons were largely preserved in CORT-treated mice, as evidenced by the unchanged connection probability and basal synaptic properties (Figure 10, 11). Moreover, there were also no sex-dependent effects of chronic CORT treatment on synaptic inhibition in either of the two IN types (Figure 10,11). This lack of effects was likely not due to the lack of a stress-related phenotype, as CORT-treated mice showed clear physiological and behavioral signs of stress, such as reduced weight gain and increased anxiety-like behavior in the open field (Figure 9), in line with previous findings (Moda-Sava et al., 2019). A potential confounding factor could have been the additional treatment of mice with low doses of EtOH as vehicle, which can enhance GABA function (Roberto et al., 2003). However, the final concentration was only $1 \%$. Moreover, the basal synaptic properties of GABAergic synapses made by both $\mathrm{PV}+$ and $\mathrm{CB} 1+\mathrm{INs}$ in $\mathrm{EtOH}$-treated vehicle controls were highly comparable to those seen in untreated, naïve mice of similar age (Figure $7,10,11$ ), thus rather ruling out an additional effect of $\mathrm{EtOH}$ that could have masked possible CORT effects on synaptic function. Together, these findings suggest that basal synaptic transmission at GABAergic synapses made by prefrontal PV+ and $\mathrm{CB} 1+\mathrm{INs}$ is highly resistant to the effects of stress-related glucocorticoid signaling during adolescence. However, the present findings do not rule out possible CORT-induced changes at other IN types or other aspects of IN functionality, such as intrinsic excitability.

In sharp contrast, a wealth of studies consistently showed that chronic exposure to stress or glucocorticoids induces severe structural and functional impairments at the level of excitatory synapses in mPFC pyramidal neurons (Wellman, 2001; Radley et al., 2006; Cerqueira et al., 2007; Yuen et al., 2012; for review see: Popoli et al., 2011). Indeed, using a similar protocol as employed here, Moda-Sava et al. (2019) demonstrated severe excitatory synapse loss in mPFC pyramidal neurons in mice chronically treated with CORT, thereby leading to profound prefrontal circuit dysfunction and depression- and anxiety-like behavior. It has been shown that changes in the excitatory glutamatergic drive onto cortical principal neurons are compensated - or balanced - by corresponding adaptations in the strength of their local inhibitory inputs (Okun and Lampl, 2008; Xue et al., 2014). This so-called excitation/inhibition (E/I) balance is an equilibrating, homeostatic type of control that is thought to enable optimal network stability and functionality, with aberrations in either direction being implicated in pathological disease states (Isaacson and Scanziani, 2011; Turrigiano, 2011; Ferguson and Gao, 2018). In visual cortex, this $E / l$ balance is largely mediated by homeostatic adaptations in the strength of perisomatic GABAergic synapses made by PV+ INs (Xue et al., 2014). Thus, the lack of CORTinduced changes at GABAergic synapses made by PV+ and CB1+ INs in the mPFC, despite the presence of a severe loss of excitatory inputs onto pyramidal neurons (Moda-Sava et al., 2019), could possibly indicate a glucocorticoid-induced dysfunction in the homeostatic 
plasticity of inhibitory synapses to balance changes in excitation. This impaired homeostatic inhibitory plasticity may consequently lead to prefrontal hypoactivity and E/I imbalance, thereby causing anxiety- and depression-like phenotypes (Page and Coutellier, 2019). It would be therefore interesting to assess possible CORT-induced changes in the prefrontal E/I balance, e.g. by recording AMPA/GABA ratios in pyramidal neurons. Moreover, it would be of value to disentangle the individual contributions of $\mathrm{PV}+$ and $\mathrm{CB} 1+\mathrm{INs}$ in maintaining such $\mathrm{E} / \mathrm{l}$ balance. Finally, future studies should also examine possible homeostatic adaptations at other levels of IN functionality that could serve to preserve prefrontal network activity in the face of glucocorticoid-induced dysfunction, such as changes in the intrinsic excitability or excitatory glutamatergic drive (Turrigiano, 2011).

\subsubsection{Chronic corticosterone treatment during adolescence impairs endocannabinoid-mediated retrograde control of synaptic inhibition by prefrontal CB1+ interneurons}

Strikingly, I found that chronic CORT treatment during adolescence led to impaired DSI at GABAergic synapses made by CB1+ INs in mPFC, indicating a deficiency in eCB-dependent inhibitory synaptic plasticity (Figure 11). Different mechanisms could account for the observed deficit in eCB-dependent DSI at these synapses.

The impairment could have been due to occlusion. DSI is mediated by the phasic retrograde release of 2-AG (Tanimura et al., 2010; Gao et al., 2010). Previous studies showed that acute stress or treatment with glucocorticoids can increase 2-AG signaling in the mPFC (Hill et al., 2011b; McReynolds et al., 2018). Thus, such tonic signaling could have occluded any additional effects of depolarization-induced phasic 2-AG signaling and thus DSI. However, the impairment in DSI observed here was likely not mediated by an occlusive mechanism via glucocorticoid-induced tonic eCB signaling for several reasons. Firstly, chronic stress exposure does not consistently elevate tonic 2-AG signaling in the mPFC (Hill et al., 2005; 2008; Hill et al., 2010; Rademacher et al., 2008; Gray et al., 2016). Secondly, the deficiency in DSI was highly selective, while basal synaptic transmission was completely preserved at GABAergic synapses made by prefrontal CB1+ INs (Figure 11). By contrast, occlusion of DSI via glucocorticoid-induced tonic eCB signaling should have also been accompanied by a reduced strength of basal transmission at these synapses. Thus, the observed deficit likely reflected a specific deficit in phasic 2-AG signaling. Of note, I observed a substantial heterogeneity in DSI between individual synapses in CORT-treated mice. This variability should be interpreted with caution, but possibly indicates that CORT treatment impaired DSI only in a subset of GABAergic synapses. Together, these data suggest that chronic stress-related glucocorticoid signaling induces a selective impairment in phasic eCB-dependent retrograde control over synaptic inhibition through prefrontal $\mathrm{CB} 1+$ INs. This impairment in inhibitory plasticity may reduce the ability of postsynaptic pyramidal neurons to down-regulate their inhibitory synaptic 
inputs during excitatory activity and could thus lead to a dysfunctional disinhibitory gating of prefrontal circuit activity. Such a dysfunction in eCB-mediated disinhibitory gating is consistent with the concept of prefrontal hypoactivity introduced before (see section 4.4.2) and may impair the recruitment of stress-relevant prefrontal microcircuits that serve to promote adaptive coping strategies during exposure to stress (McLaughlin et al., 2014). Such CORT-induced changes in eCB-dependent disinhibitory gating should be further investigated using EPSP-spike coupling recordings in prefrontal pyramidal neurons.

Interestingly, evidence suggests that chronic stress exerts opposing effects on eCB-dependent disinhibitory gating in different regions of the brain. In the hippocampus, similar stress-induced deficits in DSI have been seen as in the present study. Hu et al. (2011) found that eCBdependent DSI of carbachol-induced SIPSCs was impaired in hippocampal CA1 pyramidal neurons following 21 days of chronic restraint stress. Moreover, Zhong et al. (2014) demonstrated impaired DSI of electrically evoked IPSCs in CA1 pyramidal neurons following five weeks of chronic unpredictable stress in adult male mice. This deficit seemed to be mediated by enhanced 2-AG degradation. Indeed, chronic inhibition of the 2-AG degrading enzyme MAGL restored DSI and rescued stress-induced depression-like phenotypes in a CB1 receptor-dependent manner (Zhong et al., 2014). It would be therefore interesting to examine whether restoring eCB-dependent DSI at synapses made by prefrontal CB1+ INs would rescue the increased anxiety-like behavior in CORT-treated mice. By contrast, opposite effects have been reported in the amygdala, an important subcortical region implicated in affective processing and the regulation of stress and anxiety (Roozendaal et al., 2009). Patel et al. (2009) found that eCB-dependent DSI was prolonged in principle neurons of the basolateral amygdala (BLA) following 10 days of restraint stress in male adolescent mice due to enhanced 2-AG signaling. Using the same protocol, Sumislawski et al. (2011) showed that this stressinduced enhancement of 2-AG signaling also reversibly gated the induction of eCB-dependent LTDi in BLA neurons, thus leading to a facilitation of long-term depression of inhibitory inputs. These findings suggest that chronic stress-induced glucocorticoid signaling has opposing effects on eCB-dependent disinhibitory gating of circuit activity in amygdala, hippocampus, and mPFC. It facilitates disinhibitory gating in amygdala circuits but impairs disinhibitory gating in hippocampal and prefrontal circuits. Importantly, these regions have opposing functions in the regulation of the stress response. While hippocampus and mPFC exert a negative feedback function serving to down-regulate HPA axis activity in order to enable stress adaptation, the amygdala exerts a positive feedback function, further enhancing HPA axis activity following exposure to stress (Herman et al., 2016). Therefore, these findings favor a working model according to which the opposing effects of chronic stress on eCB-dependent inhibitory plasticity may impair the stress-buffering capacity of hippocampal and prefrontal circuits, while 
boosting the stress-potentiating function of the amygdala, ultimately leading to a homeostatic dysregulation of HPA axis activity.

Together, the present results point to a selective glucocorticoid-induced deficiency in eCBdependent retrograde control of synaptic inhibition through CB1+ INs in the prefrontal cortex, thus implicating them as a major target for stress-induced dysfunctions in eCB signaling. The impaired plasticity at GABAergic synapses made by CB1+ INs may lead to a dysfunctional disinhibitory gating of prefrontal circuit activity, possibly resulting in impaired prefrontal stress regulation and cognitive performance. Such impaired eCB-dependent inhibitory synaptic plasticity could increase the risk of developing psychiatric disorders characterized by stressinduced cognitive dysfunction and mood disturbances, such as schizophrenia, major depression, and anxiety disorders.

\subsection{Conclusions and Outlook}

In this study, I demonstrated that the expression of cell type-specific inhibitory network motifs is strongly shaped by cortical hierarchy. While PV+ INs represent the major source of perisomatic inhibition in primary sensory cortices such as $\mathrm{S} 1$, inhibitory control is largely balanced between PV+ and CB1+ INs in the mPFC, a higher-order associative cortical structure linked to cognitive processing (Figure 12A, B). These results challenge the traditional view of a dominant function of $\mathrm{PV}+\mathrm{INs}$ in network refinement throughout neocortex and point to an increased importance of $\mathrm{CB} 1+\mathrm{INs}$ in regulating prefrontal network activity linked to cognitive control and mood regulation. In support of this, I further showed that chronic stressrelated glucocorticoid signaling during adolescence induces a selective deficiency in eCBdependent retrograde suppression of synaptic inhibition through $\mathrm{CB} 1+$ INs in the mPFC (Figure 12C). These results implicate $\mathrm{CB} 1+\mathrm{INs}$ as a major cellular target for stress-induced dysfunctions in eCB signaling and point to a specific impairment in eCB-dependent disinhibitory gating of prefrontal circuit activity as a correlate of stress-related phenotypes. Together, these findings suggest CB1+ INs as a promising new candidate for studying eCBdependent processes with relevance to stress-related psychiatric diseases, such as schizophrenia, major depression, and anxiety disorders.

Many open questions remain. Do PV+ and CB1+ INs innervate the same target neurons or do they form distinct prefrontal microcircuits? Is the balanced mode of inhibitory control between $\mathrm{PV}+$ and $\mathrm{CB} 1+\mathrm{INs}$ also evident in other higher-order cortical structures, such as the entorhinal cortex? What is the functional role of $\mathrm{CB} 1+\mathrm{INs}$ in stress-induced prefrontal circuit dysfunction? Does the glucocorticoid-induced deficit in eCB-dependent plasticity at GABAergic synapses of $\mathrm{CB} 1+$ INs lead to impaired gating of prefrontal circuit activity in vivo and is it causally linked to stress-induced behavioral alterations? How permanent are the synaptic changes and can they also be seen following exposure to physical and psychosocial stress? Are the deficits specific to GABAergic synapses? What are the mechanisms leading to the glucocorticoid-induced 
impairment in eCB-dependent DSI? Is it caused by changes in the number or affinity of presynaptic $\mathrm{CB} 1$ receptors or by changes in the number or functionality of 2-AG synthesizing or degrading enzymes (Figure 12D)? In light of the opposing effects of chronic glucocorticoid signaling on eCB-dependent inhibitory plasticity in prefrontal and amygdala circuits, it would be worthwhile to contrast CORT-induced synaptic changes in the prefrontal cortex with those in the amygdala. Answering these questions will help us gain a deeper understanding into eCB-dependent control of cortical inhibition and the pathomechanisms of stress-related psychiatric disorders.

A

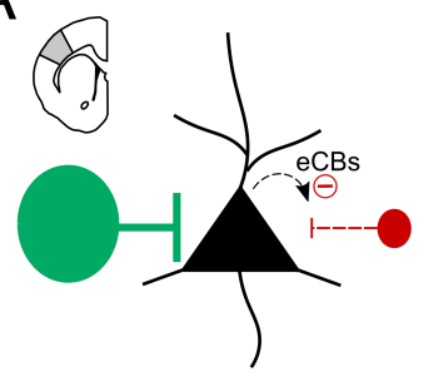

D

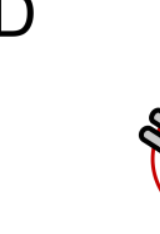

$\mathrm{B}$
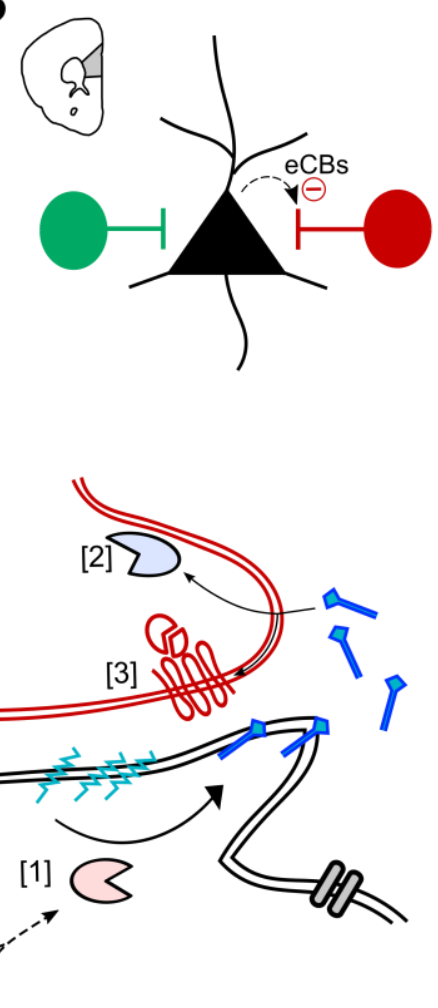

C

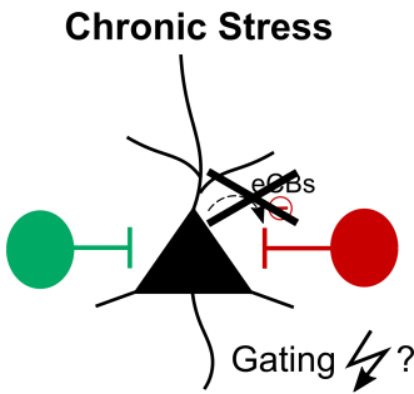

00 VGCC

$00 \mathrm{GABA}_{A} \mathrm{R}$
Q气్ CB1R

ß DAGL

$\sum M A G L$

$\Longrightarrow 2$-AG

$-\mathrm{NW}$ lipid precursor

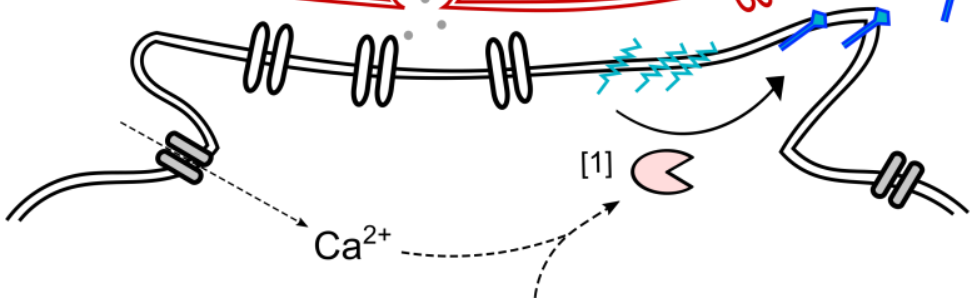

Figure 12: A cortical hierarchy for endocannabinoid-regulated stress-sensitive inhibitory circuits in neocortex. A) In primary sensory cortices such as S1, inhibitory control of glutamatergic pyramidal neurons (black) is dominated by PV+ INs (green), while eCB-sensitive CB1+ INs (red) only serve a secondary function. B) By contrast, inhibitory control is balanced between $\mathrm{PV}+$ and $\mathrm{CB} 1+\mathrm{INs}$ in higher-order associative cortices such as the mPFC. C) Chronic glucocorticoid-mediated stress does not affect basal inhibitory drive, but selectively impairs retrograde eCB-mediated suppression of synaptic transmission at GABAergic synapses made by CB1+ INs in the mPFC, possibly leading to dysfunctional disinhibitory gating of prefrontal circuit activity and thus impaired stress regulation. D) Possible mechanisms leading to the chronic stress-induced deficit in eCB-mediated retrograde suppression of synaptic inhibition. $\mathrm{Ca}^{2+}$ influx during postsynaptic depolarization of pyramidal neurons activates DAGL, which triggers the synthesis of 2-AG from lipid precursors. 2-AG is then released into the synaptic cleft to activate presynaptic CB1Rs. CB1R activation inhibits VGCCs and thereby transiently suppresses GABA release. $2-A G$ is catabolized at the presynaptic site by the degrading enzyme MAGL. Chronic stress could disrupt this eCBdependent retrograde control of inhibition [1] by impairing DAGL-mediated 2-AG synthesis, [2] by enhancing MAGLmediated 2-AG degradation, or [3] by reducing the number or affinity of CB1Rs. These three mechanisms are not mutually exclusive, but could principally operate in parallel. Abbreviations: $\mathrm{eCB}=$ endocannabinoid; $\mathrm{CB} 1 \mathrm{R}=$ cannabinoid type-1 receptor; $\mathrm{DAGL}=$ diacylglycerol lipase; $\mathrm{MAGL}=$ monoacylglycerol lipase; $2-\mathrm{AG}=2$ arachidonoylglycerol; VGCC $=$ voltage-gated calcium $\mathrm{Ca}^{2+}$ channel; GABA $A R=G A B A_{A}$ receptor. Panel $D$ has been adapted from Kano et al. (2009). 


\section{References}

Alcamí P, Pereda AE (2019) Beyond plasticity: the dynamic impact of electrical synapses on neural circuits. Nat Rev Neurosci 20:253-271.

Ali AB (2007) Presynaptic inhibition of GABAA receptor-mediated unitary IPSPs by cannabinoid receptors at synapses between CCK-positive interneurons in rat hippocampus. J Neurophysiol 98:861-869.

Ali AB, Thomson AM (2008) Synaptic alpha 5 subunit-containing GABAA receptors mediate IPSPs elicited by dendrite-preferring cells in rat neocortex. Cereb Cortex 18:1260-1271.

Andersen SL, Tomada A, Vincow ES, Valente E, Polcari A, Teicher MH (2008) Preliminary evidence for sensitive periods in the effect of childhood sexual abuse on regional brain development. J Neuropsychiatry Clin Neurosci 20:292-301.

Arnsten AFT (2009) Stress signalling pathways that impair prefrontal cortex structure and function. Nat Rev Neurosci 10:410-422.

Banks MI, White JA, Pearce RA (2000) Interactions between distinct GABAA circuits in hippocampus. Neuron 25:449-457.

Bodor AL, Katona I, Nyíri G, Mackie K, Ledent C, Hájos N, Freund TF (2005) Endocannabinoid signaling in rat somatosensory cortex: laminar differences and involvement of specific interneuron types. J Neurosci 25:6845-6856.

Brown VJ, Bowman EM (2002) Rodent models of prefrontal cortical function. Trends Neurosci 25:340-343.

Caballero A, Flores-Barrera E, Cass DK, Tseng KY (2014) Differential regulation of parvalbumin and calretinin interneurons in the prefrontal cortex during adolescence. Brain Struct Funct 219:395-406.

Caballero A, Tseng KY (2016) GABAergic function as a limiting factor for prefrontal maturation during adolescence. Trends Neurosci 39:441-448.

Caspi A, Sugden K, Moffitt TE, Taylor A, Craig IW, Harrington H, McClay J, Mill J, Martin J, Braithwaite A, Poulton R (2003) Influence of life stress on depression: moderation by a polymorphism in the 5-HTT gene. Science 301:386-389.

Cass DK, Flores-Barrera E, Thomases DR, Vital WF, Caballero A, Tseng KY (2014) CB1 cannabinoid receptor stimulation during adolescence impairs the maturation of GABA function in the adult rat prefrontal cortex. Mol Psychiatry 19:536-543.

Castillo PE, Younts TJ, Chávez AE, Hashimotodani Y (2012) Endocannabinoid signaling and synaptic function. Neuron 76:70-81.

Cea-del Rio CA, Lawrence JJ, Tricoire L, Erdelyi F, Szabo G, McBain CJ (2010) M3 muscarinic acetylcholine receptor expression confers differential cholinergic modulation to neurochemically distinct hippocampal basket cell subtypes. J Neurosci 30:6011-6024.

Cerqueira JJ, Mailliet F, Almeida OFX, Jay TM, Sousa N (2007) The prefrontal cortex as a key target of the maladaptive response to stress. J Neurosci 27:2781-2787.

Chattopadhyaya B, Di Cristo G, Higashiyama H, Knott GW, Kuhlman SJ, Welker E, Huang ZJ (2004) Experience and activity-dependent maturation of perisomatic GABAergic innervation in primary visual cortex during a postnatal critical period. J Neurosci 24:95989611. 
Chevaleyre V, Castillo PE (2003) Heterosynaptic LTD of hippocampal GABAergic synapses: A novel role of endocannabinoids in regulating excitability 38:461-472.

Chevaleyre V, Castillo PE (2004) Endocannabinoid-mediated metaplasticity in the hippocampus. Neuron 43:871-881.

Chevaleyre V, Heifets BD, Kaeser PS, Südhof TC, Purpura DP, Castillo PE (2007) Endocannabinoid-mediated long-term plasticity requires CAMP/PKA signaling and RIM1alpha. Neuron 54:801-812.

Chevaleyre V, Takahashi KA, Castillo PE (2006) Endocannabinoid-mediated synaptic plasticity in the CNS. Annu Rev Neurosci 29:37-76.

Chiba S, Numakawa T, Ninomiya M, Richards MC, Wakabayashi C, Kunugi H (2012) Chronic restraint stress causes anxiety- and depression-like behaviors, downregulates glucocorticoid receptor expression, and attenuates glutamate release induced by brainderived neurotrophic factor in the prefrontal cortex. Prog Neuropsychopharmacol Biol Psychiatry 39:112-119.

Chiu CQ, Puente N, Grandes P, Castillo PE (2010) Dopaminergic modulation of endocannabinoid-mediated plasticity at GABAergic synapses in the prefrontal cortex. $J$ Neurosci 30:7236-7248.

Choi K, Le T, McGuire J, Xing G, Zhang L, Li H, Parker CC, Johnson LR, Ursano RJ (2012) Expression pattern of the cannabinoid receptor genes in the frontal cortex of mood disorder patients and mice selectively bred for high and low fear. J Psychiatr Res 46:882-889.

Choleris E, Thomas AW, Kavaliers M, Prato FS (2001) A detailed ethological analysis of the mouse open field test: effects of diazepam, chlordiazepoxide and an extremely low frequency pulsed magnetic field. Neuroscience \& Biobehavioral Reviews 25:235-260.

Chung DW, Wills ZP, Fish KN, Lewis DA (2017) Developmental pruning of excitatory synaptic inputs to parvalbumin interneurons in monkey prefrontal cortex. Proc Natl Acad Sci U S A 114:E629-E637.

Collins DP, Anastasiades PG, Marlin JJ, Carter AG (2018) Reciprocal circuits linking the prefrontal cortex with dorsal and ventral thalamic nuclei. Neuron 98:366-379.e4.

Conn PJ, Pin JP (1997) Pharmacology and functions of metabotropic glutamate receptors. Annu Rev Pharmacol Toxicol 37:205-237.

Cook SC, Wellman CL (2004) Chronic stress alters dendritic morphology in rat medial prefrontal cortex. J Neurobiol 60:236-248.

Cope DW, Maccaferri G, Márton LF, Roberts JDB, Cobden PM, Somogyi P (2002) Cholecystokinin-immunopositive basket and Schaffer collateral-associated interneurones target different domains of pyramidal cells in the CA1 area of the rat hippocampus. Neuroscience 109:63-80.

Czéh B, Vardya I, Varga Z, Febbraro F, Csabai D, Martis L-S, Højgaard K, Henningsen K, Bouzinova EV, Miseta A, Jensen K, Wiborg O (2018) Long-term stress disrupts the structural and functional integrity of GABAergic neuronal networks in the medial prefrontal cortex of rats. Front Cell Neurosci 12:148.

Dobrunz LE, Stevens CF (1997) Heterogeneity of release probability, facilitation, and depletion at central synapses. Neuron 18:995-1008. 
Douglas RJ, Martin KAC (2004) Neuronal circuits of the neocortex. Annu Rev Neurosci 27:419-451.

Duncan GE, Johnson KB, Breese GR (1993) Topographic patterns of brain activity in response to swim stress: assessment by 2-deoxyglucose uptake and expression of Fos-like immunoreactivity. J. Neurosci. 13:3932-3943.

Egertová M, Cravatt BF, Elphick MR (2003) Comparative analysis of fatty acid amide hydrolase and CB1 cannabinoid receptor expression in the mouse brain: evidence of a widespread role for fatty acid amide hydrolase in regulation of endocannabinoid signaling. Neuroscience 119:481-496.

Egertová M, Giang DK, Cravatt BF, Elphick MR (1998) A new perspective on cannabinoid signalling: complementary localization of fatty acid amide hydrolase and the CB1 receptor in rat brain. Proc Biol Sci 265:2081-2085.

Eggan SM, Hashimoto T, Lewis DA (2008) Reduced cortical cannabinoid 1 receptor messenger RNA and protein expression in schizophrenia. Arch Gen Psychiatry 65:772784.

Eggan SM, Lewis DA (2007) Immunocytochemical distribution of the cannabinoid CB1 receptor in the primate neocortex: a regional and laminar analysis. Cereb Cortex 17:175191.

Eggan SM, Stoyak SR, Verrico CD, Lewis DA (2010) Cannabinoid CB1 receptor immunoreactivity in the prefrontal cortex: Comparison of schizophrenia and major depressive disorder. Neuropsychopharmacology 35:2060-2071.

Farrant M, Nusser Z (2005) Variations on an inhibitory theme: phasic and tonic activation of GABA(A) receptors. Nat Rev Neurosci 6:215-229.

Férézou I, Cauli B, Hill EL, Rossier J, Hamel E, Lambolez B (2002) 5-HT3 receptors mediate serotonergic fast synaptic excitation of neocortical vasoactive intestinal peptide/cholecystokinin interneurons. J Neurosci 22:7389-7397.

Ferguson BR, Gao W-J (2018) PV interneurons: Critical regulators of E/l balance for prefrontal cortex-dependent behavior and psychiatric disorders. Front Neural Circuits 12:37.

Fino E, Packer AM, Yuste R (2013) The logic of inhibitory connectivity in the neocortex. Neuroscientist 19:228-237.

Földy C, Lee SY, Szabadics J, Neu A, Soltesz I (2007) Cell type-specific gating of perisomatic inhibition by cholecystokinin. Nat Neurosci 10:1128-1130.

Földy C, Neu A, Jones MV, Soltesz I (2006) Presynaptic, activity-dependent modulation of cannabinoid type 1 receptor-mediated inhibition of GABA release. J Neurosci 26:14651469.

Fortin DA, Levine ES (2007) Differential effects of endocannabinoids on glutamatergic and GABAergic inputs to layer 5 pyramidal neurons. Cereb Cortex 17:163-174.

Fortin DA, Trettel J, Levine ES (2004) Brief trains of action potentials enhance pyramidal neuron excitability via endocannabinoid-mediated suppression of inhibition. J Neurophysiol 92:2105-2112.

Freund TF (2003) Interneuron Diversity series: Rhythm and mood in perisomatic inhibition. Trends Neurosci 26:489-495. 
Freund TF, Katona I (2007) Perisomatic inhibition. Neuron 56:33-42.

Gabernet L, Jadhav SP, Feldman DE, Carandini M, Scanziani M (2005) Somatosensory integration controlled by dynamic thalamocortical feed-forward inhibition. Neuron 48:315327.

Galarreta M, Erdélyi F, Szabó G, Hestrin S (2004) Electrical coupling among irregular-spiking GABAergic interneurons expressing cannabinoid receptors. J Neurosci 24:9770-9778.

Galarreta M, Erdélyi F, Szabó G, Hestrin S (2008) Cannabinoid sensitivity and synaptic properties of 2 GABAergic networks in the neocortex. Cereb Cortex 18:2296-2305.

Galarreta M, Hestrin S (1999) A network of fast-spiking cells in the neocortex connected by electrical synapses. Nature 402:72-75.

Gao $Y$ et al. (2010) Loss of retrograde endocannabinoid signaling and reduced adult neurogenesis in diacylglycerol lipase knock-out mice. J Neurosci 30:2017-2024.

Gee DG, Casey BJ (2015) The impact of developmental timing for stress and recovery. Neurobiol Stress 1:184-194.

Gerdeman GL, Ronesi J, Lovinger DM (2002) Postsynaptic endocannabinoid release is critical to long-term depression in the striatum. Nat Neurosci 5:446-451.

Ghosal S, Duman CH, Liu R-J, Wu M, Terwilliger R, Girgenti MJ, Wohleb E, Fogaca MV, Teichman EM, Hare B, Duman RS (2020) Ketamine rapidly reverses stress-induced impairments in GABAergic transmission in the prefrontal cortex in male rodents. Neurobiol Dis 134:104669.

Gilbert CD, Li W (2013) Top-down influences on visual processing. Nat Rev Neurosci 14:350363.

Gilbert CD, Wiesel TN (1979) Morphology and intracortical projections of functionally characterised neurones in the cat visual cortex. Nature 280:120-125.

Glass M, Faull RLM, Dragunow M (1997) Cannabinoid receptors in the human brain: a detailed anatomical and quantitative autoradiographic study in the fetal, neonatal and adult human brain. Neuroscience 77:299-318.

Glickfeld LL, Scanziani M (2006) Distinct timing in the activity of cannabinoid-sensitive and cannabinoid-insensitive basket cells. Nat Neurosci 9:807-815.

Gray JM, Wilson CD, Lee TTY, Pittman QJ, Deussing JM, Hillard CJ, McEwen BS, Schulkin J, Karatsoreos IN, Patel S, Hill MN (2016) Sustained glucocorticoid exposure recruits corticolimbic $\mathrm{CRH}$ signaling to modulate endocannabinoid function. Psychoneuroendocrinology 66:151-158.

Gregoriou GG, Rossi AF, Ungerleider LG, Desimone R (2014) Lesions of prefrontal cortex reduce attentional modulation of neuronal responses and synchrony in V4. Nat Neurosci 17:1003-1011.

Gulyas AI, Cravatt BF, Bracey MH, Dinh TP, Piomelli D, Boscia F, Freund TF (2004) Segregation of two endocannabinoid-hydrolyzing enzymes into pre- and postsynaptic compartments in the rat hippocampus, cerebellum and amygdala. Eur J Neurosci 20:441458. 
Hájos N, Katona I, Naiem SS, MacKie K, Ledent C, Mody I, Freund TF (2000) Cannabinoids inhibit hippocampal GABAergic transmission and network oscillations. Eur J Neurosci 12:3239-3249.

Harris KD, Shepherd GM (2015) The neocortical circuit: themes and variations. Nat Neurosci 18:170-181.

Hashimotodani Y, Ohno-Shosaku T, Kano M (2007) Endocannabinoids and synaptic function in the CNS. Neuroscientist 13:127-137.

Hefft S, Jonas P (2005) Asynchronous GABA release generates long-lasting inhibition at a hippocampal interneuron-principal neuron synapse. Nat Neurosci 8:1319-1328.

Heidbreder CA, Groenewegen HJ (2003) The medial prefrontal cortex in the rat: evidence for a dorso-ventral distinction based upon functional and anatomical characteristics. Neurosci Biobehav Rev 27:555-579.

Heifets BD, Chevaleyre V, Castillo PE (2008) Interneuron activity controls endocannabinoidmediated presynaptic plasticity through calcineurin. Proc Natl Acad Sci U S A 105:1025010255.

Hensch TK (2005) Critical period plasticity in local cortical circuits. Nat Rev Neurosci 6:877888.

Hensch TK, Fagiolini M, Mataga N, Stryker MP, Baekkeskov S, Kash SF (1998) Local GABA circuit control of experience-dependent plasticity in developing visual cortex. Science 282:1504-1508.

Herkenham M, Lynn AB, Johnson, Melvin LS, Costa BR de, Rice KC (1991) Characterization and localization of cannabinoid receptors in rat brain: a quantitative in vitro autoradiographic study. J. Neurosci. 11:563-583.

Herkenham M, Lynn AB, Little MD, Johnson MR, Melvin LS, Costa BR de, Rice KC (1990) Cannabinoid receptor localization in brain. Proc Natl Acad Sci U S A 87:1932-1936.

Herman JP, McKlveen JM, Ghosal S, Kopp B, Wulsin A, Makinson R, Scheimann J, Myers B (2016) Regulation of the hypothalamic-pituitary-adrenocortical stress response. Compr Physiol 6:603-621.

Hill EL, Gallopin T, Férézou I, Cauli B, Rossier J, Schweitzer P, Lambolez B (2007) Functional CB1 receptors are broadly expressed in neocortical GABAergic and glutamatergic neurons. J Neurophysiol 97:2580-2589.

Hill MN, Carrier EJ, McLaughlin RJ, Morrish AC, Meier SE, Hillard CJ, Gorzalka BB (2008) Regional alterations in the endocannabinoid system in an animal model of depression: effects of concurrent antidepressant treatment. J Neurochem 106:2322-2336.

Hill MN, Hillard CJ, McEwen BS (2011a) Alterations in corticolimbic dendritic morphology and emotional behavior in cannabinoid CB1 receptor-deficient mice parallel the effects of chronic stress. Cereb Cortex 21:2056-2064.

Hill MN, McLaughlin RJ, Bingham B, Shrestha L, Lee TTY, Gray JM, Hillard CJ, Gorzalka BB, Viau V (2010) Endogenous cannabinoid signaling is essential for stress adaptation. Proc Natl Acad Sci U S A 107:9406-9411.

Hill MN, McLaughlin RJ, Pan B, Fitzgerald ML, Roberts CJ, Lee TT-Y, Karatsoreos IN, Mackie K, Viau V, Pickel VM, McEwen BS, Liu Q-s, Gorzalka BB, Hillard CJ (2011b) Recruitment 
of prefrontal cortical endocannabinoid signaling by glucocorticoids contributes to termination of the stress response. J Neurosci 31:10506-10515.

Hill MN, Patel S, Carrier EJ, Rademacher DJ, Ormerod BK, Hillard CJ, Gorzalka BB (2005) Downregulation of endocannabinoid signaling in the hippocampus following chronic unpredictable stress. Neuropsychopharmacology 30:508-515.

Hippenmeyer S, Vrieseling E, Sigrist M, Portmann T, Laengle C, Ladle DR, Arber S (2005) A developmental switch in the response of DRG neurons to ETS transcription factor signaling. PLoS Biol 3:e159.

Holmgren C, Harkany T, Svennenfors B, Zilberter Y (2003) Pyramidal cell communication within local networks in layer 2/3 of rat neocortex. J Physiol (Lond) 551:139-153.

Hsia AY, Malenka RC, Nicoll RA (1998) Development of excitatory circuitry in the hippocampus. J Neurophysiol 79:2013-2024.

Hu W, Zhang M, Czéh B, Zhang W, Flügge G (2011) Chronic restraint stress impairs endocannabinoid mediated suppression of GABAergic signaling in the hippocampus of adult male rats. Brain Res Bull 85:374-379.

Huang S, Gu Y, Quinlan EM, Kirkwood A (2010) A refractory period for rejuvenating GABAergic synaptic transmission and ocular dominance plasticity with dark exposure. J Neurosci 30:16636-16642.

Huang ZJ, Kirkwood A, Pizzorusso T, Porciatti V, Morales B, Bear MF, Maffei L, Tonegawa S (1999) BDNF regulates the maturation of inhibition and the critical period of plasticity in mouse visual cortex. Cell 98:739-755.

Hungund BL, Vinod KY, Kassir SA, Basavarajappa BS, Yalamanchili R, Cooper TB, Mann JJ, Arango V (2004) Upregulation of CB1 receptors and agonist-stimulated [35S]GTPgammaS binding in the prefrontal cortex of depressed suicide victims. Mol Psychiatry 9:184-190.

Huttenlocher PR, Dabholkar AS (1997) Regional differences in synaptogenesis in human cerebral cortex. J Comp Neurol 387:167-178.

Isaacson JS, Scanziani M (2011) How inhibition shapes cortical activity. Neuron 72:231-243.

Jessell TM, Kandel ER (1993) Synaptic transmission: A bidirectional and self-modifiable form of cell-cell communication. Cell 72:1-30.

Jiang $B$, Huang S, Pasquale R de, Millman D, Song L, Lee H-K, Tsumoto T, Kirkwood A (2010a) The maturation of GABAergic transmission in visual cortex requires endocannabinoid-mediated LTD of inhibitory inputs during a critical period. Neuron 66:248259.

Jiang B, Sohya K, Sarihi A, Yanagawa Y, Tsumoto T (2010b) Laminar-specific maturation of GABAergic transmission and susceptibility to visual deprivation are related to endocannabinoid sensitivity in mouse visual cortex. J Neurosci 30:14261-14272.

Jin H, Wu H, Osterhaus G, Wei J, Davis K, Di S, Floor E, Hsu C-C, Kopke RD, Wu J-Y (2003) Demonstration of functional coupling between gamma-aminobutyric acid (GABA) synthesis and vesicular GABA transport into synaptic vesicles. Proc Natl Acad Sci U S A 100:42934298.

Kano M, Ohno-Shosaku T, Hashimotodani Y, Uchigashima M, Watanabe M (2009) Endocannabinoid-mediated control of synaptic transmission. Physiol Rev 89:309-380. 
Karatsoreos IN, Bhagat SM, Bowles NP, Weil ZM, Pfaff DW, McEwen BS (2010) Endocrine and physiological changes in response to chronic corticosterone: a potential model of the metabolic syndrome in mouse. Endocrinology 151:2117-2127.

Karson MA, Tang A-H, Milner TA, Alger BE (2009) Synaptic cross talk between perisomatictargeting interneuron classes expressing cholecystokinin and parvalbumin in hippocampus. J Neurosci 29:4140-4154.

Katona I, Sperlágh B, Sík A, Käfalvi A, Vizi ES, Mackie K, Freund TF (1999) Presynaptically located CB1 cannabinoid receptors regulate GABA release from axon terminals of specific hippocampal interneurons. J Neurosci 19:4544-4558.

Katona I, Urbán GM, Wallace M, Ledent C, Jung K-M, Piomelli D, Mackie K, Freund TF (2006) Molecular composition of the endocannabinoid system at glutamatergic synapses. $J$ Neurosci 26:5628-5637.

Kawaguchi Y (1997) Selective cholinergic modulation of cortical GABAergic cell subtypes. J Neurophysiol 78:1743-1747.

Kepecs A, Fishell G (2014) Interneuron cell types are fit to function. Nature 505:318-326.

Keros S, Hablitz JJ (2005) Subtype-specific GABA transporter antagonists synergistically modulate phasic and tonic GABAA conductances in rat neocortex. J Neurophysiol 94:2073-2085.

Kneussel M, Brandstätter JH, Laube B, Stahl S, Müller U, Betz H (1999) Loss of postsynaptic GABA A receptor clustering in gephyrin-deficient mice. J. Neurosci. 19:9289-9297.

Kreitzer AC, Regehr WG (2001) Retrograde inhibition of presynaptic calcium influx by endogenous cannabinoids at excitatory synapses onto Purkinje cells. Neuron 29:717-721.

Krueger-Burg D, Papadopoulos T, Brose N (2017) Organizers of inhibitory synapses come of age. Curr Opin Neurobiol 45:66-77.

Kucewicz MT, Tricklebank MD, Bogacz R, Jones MW (2011) Dysfunctional prefrontal cortical network activity and interactions following cannabinoid receptor activation. J Neurosci $31: 15560-15568$.

Kuhlman SJ, Olivas ND, Tring E, Ikrar T, Xu X, Trachtenberg JT (2013) A disinhibitory microcircuit initiates critical-period plasticity in the visual cortex. Nature 501:543-546.

Laubach M, Amarante LM, Swanson K, White SR (2018) What, if anything, is rodent prefrontal cortex? eNeuro 5.

Le Magueresse C, Monyer H (2013) GABAergic interneurons shape the functional maturation of the cortex. Neuron 77:388-405.

Lee S, Hjerling-Leffler J, Zagha E, Fishell G, Rudy B (2010a) The largest group of superficial neocortical GABAergic interneurons expresses ionotropic serotonin receptors. J Neurosci 30:16796-16808.

Lee S, Kruglikov I, Huang ZJ, Fishell G, Rudy B (2013) A disinhibitory circuit mediates motor integration in the somatosensory cortex. Nat Neurosci 16:1662-1670.

Lee S-H, Földy C, Soltesz I (2010b) Distinct endocannabinoid control of GABA release at perisomatic and dendritic synapses in the hippocampus. J Neurosci 30:7993-8000.

Lee S-H, Ledri M, Tóth B, Marchionni I, Henstridge CM, Dudok B, Kenesei K, Barna L, Szabó SI, Renkecz T, Oberoi M, Watanabe M, Limoli CL, Horvai G, Soltesz I, Katona I (2015) 
Multiple forms of endocannabinoid and endovanilloid signaling regulate the tonic control of GABA release. J Neurosci 35:10039-10057.

Lee SY, Földy C, Szabadics J, Soltesz I (2011) Cell-type-specific CCK2 receptor signaling underlies the cholecystokinin-mediated selective excitation of hippocampal parvalbuminpositive fast-spiking basket cells. J Neurosci 31:10993-11002.

Losonczy A, Biró AA, Nusser Z (2004) Persistently active cannabinoid receptors mute a subpopulation of hippocampal interneurons. Proc Natl Acad Sci U S A 101:1362-1367.

Lovett-Barron M, Turi GF, Kaifosh P, Lee PH, Bolze F, Sun X-H, Nicoud J-F, Zemelman BV, Sternson SM, Losonczy A (2012) Regulation of neuronal input transformations by tunable dendritic inhibition. Nat Neurosci 15:423-30, S1-3.

Lutz B, Marsicano G, Maldonado R, Hillard CJ (2015) The endocannabinoid system in guarding against fear, anxiety and stress. Nat Rev Neurosci 16:705-718.

Ma K, Xu A, Cui S, Sun M-R, Xue Y-C, Wang J-H (2016) Impaired GABA synthesis, uptake and release are associated with depression-like behaviors induced by chronic mild stress. Transl Psychiatry 6:e910.

Mackie K, Hille B (1992) Cannabinoids inhibit N-type calcium channels in neuroblastomaglioma cells. Proc Natl Acad Sci U S A 89:3825-3829.

Madisen L, Zwingman TA, Sunkin SM, Oh SW, Zariwala HA, Gu H, Ng LL, Palmiter RD, Hawrylycz MJ, Jones AR, Lein ES, Zeng H (2010) A robust and high-throughput Cre reporting and characterization system for the whole mouse brain. Nat Neurosci 13:133140.

Marco EM, Macrì S, Laviola G (2011) Critical age windows for neurodevelopmental psychiatric disorders: evidence from animal models. Neurotox Res 19:286-307.

Marsicano G, Lutz B (1999) Expression of the cannabinoid receptor CB1 in distinct neuronal subpopulations in the adult mouse forebrain. Eur J Neurosci 11:4213-4225.

Martin LA, Alger BE (1999) Muscarinic facilitation of the occurrence of depolarization-induced suppression of inhibition in rat hippocampus. Neuroscience 92:61-71.

McKlveen JM, Morano RL, Fitzgerald M, Zoubovsky S, Cassella SN, Scheimann JR, Ghosal S, Mahbod P, Packard BA, Myers B, Baccei ML, Herman JP (2016) Chronic stress increases prefrontal inhibition: A mechanism for stress-induced prefrontal dysfunction. Biol Psychiatry 80:754-764.

McKlveen JM, Myers B, Flak JN, Bundzikova J, Solomon MB, Seroogy KB, Herman JP (2013) Role of prefrontal cortex glucocorticoid receptors in stress and emotion. Biol Psychiatry 74:672-679.

McLaughlin RJ, Hill MN, Dang SS, Wainwright SR, Galea LAM, Hillard CJ, Gorzalka BB (2013) Upregulation of $\mathrm{CB}_{1}$ receptor binding in the ventromedial prefrontal cortex promotes proactive stress-coping strategies following chronic stress exposure. Behav Brain Res 237:333-337.

McLaughlin RJ, Hill MN, Gorzalka BB (2014) A critical role for prefrontocortical endocannabinoid signaling in the regulation of stress and emotional behavior. Neurosci Biobehav Rev 42:116-131.

McReynolds JR, Doncheck EM, Li Y, Vranjkovic O, Graf EN, Ogasawara D, Cravatt BF, Baker DA, Liu Q-s, Hillard CJ, Mantsch JR (2018) Stress promotes drug seeking through 
glucocorticoid-dependent endocannabinoid mobilization in the prelimbic cortex. Biol Psychiatry 84:85-94.

Meyer HS, Schwarz D, Wimmer VC, Schmitt AC, Kerr JND, Sakmann B, Helmstaedter M (2011) Inhibitory interneurons in a cortical column form hot zones of inhibition in layers 2 and 5A. Proc Natl Acad Sci U S A 108:16807-16812.

Miller EK (2000) The prefrontal cortex and cognitive control. Nat Rev Neurosci 1:59-65.

Mitchell SJ, Silver RA (2003) Shunting inhibition modulates neuronal gain during synaptic excitation. Neuron 38:433-445.

Mizoguchi K, Ishige A, Aburada M, Tabira T (2003) Chronic stress attenuates glucocorticoid negative feedback: involvement of the prefrontal cortex and hippocampus. Neuroscience 119:887-897.

Moda-Sava RN, Murdock MH, Parekh PK, Fetcho RN, Huang BS, Huynh TN, Witztum J, Shaver DC, Rosenthal DL, Alway EJ, Lopez K, Meng Y, Nellissen L, Grosenick L, Milner TA, Deisseroth K, Bito H, Kasai H, Liston C (2019) Sustained rescue of prefrontal circuit dysfunction by antidepressant-induced spine formation. Science 364:eaat8078.

Monory K, Polack M, Remus A, Lutz B, Korte M (2015) Cannabinoid CB1 receptor calibrates excitatory synaptic balance in the mouse hippocampus. J Neurosci 35:3842-3850.

Morales M, Bäckman C (2002) Coexistence of serotonin 3 (5-HT3) and CB1 cannabinoid receptors in interneurons of hippocampus and dentate gyrus. Hippocampus 12:756-764.

Morales M, Wang S-D, Diaz-Ruiz O, Jho DH-J (2004) Cannabinoid CB1 receptor and serotonin 3 receptor subunit $A(5-\mathrm{HT} 3 \mathrm{~A})$ are co-expressed in GABA neurons in the rat telencephalon. J Comp Neurol 468:205-216.

Neu A, Földy C, Soltesz I (2007) Postsynaptic origin of CB1-dependent tonic inhibition of GABA release at cholecystokinin-positive basket cell to pyramidal cell synapses in the CA1 region of the rat hippocampus. J Physiol (Lond) 578:233-247.

Neumeister A, Normandin MD, Pietrzak RH, Piomelli D, Zheng MQ, Gujarro-Anton A, Potenza MN, Bailey CR, Lin SF, Najafzadeh S, Ropchan J, Henry S, Corsi-Travali S, Carson RE, Huang $Y$ (2013) Elevated brain cannabinoid CB1 receptor availability in post-traumatic stress disorder: a positron emission tomography study. Mol Psychiatry 18:1034-1040.

Nguyen R, Venkatesan S, Binko M, Bang JY, Cajanding JD, Briggs C, Sargin D, Imayoshi I, Lambe EK, Kim JC (2020) Cholecystokinin-expressing interneurons of the medial prefrontal cortex mediate working memory retrieval. J Neurosci 40:2314-2331.

Nigro MJ, Hashikawa-Yamasaki Y, Rudy B (2018) Diversity and connectivity of layer 5 somatostatin-expressing interneurons in the mouse barrel cortex. J. Neurosci. 38:16221633.

Nudo RJ, Wise BM, SiFuentes F, Milliken GW (1996) Neural substrates for the effects of rehabilitative training on motor recovery after ischemic infarct. Science 272:1791-1794.

Nusser Z (2018) Creating diverse synapses from the same molecules. Curr Opin Neurobiol $51: 8-15$.

Nyíri G, Freund TF, Somogyi P (2001) Input-dependent synaptic targeting of alpha(2)-subunitcontaining $\mathrm{GABA}(\mathrm{A})$ receptors in synapses of hippocampal pyramidal cells of the rat. Eur J Neurosci 13:428-442. 
Ohno-Shosaku T, Maejima T, Kano M (2001) Endogenous cannabinoids mediate retrograde signals from depolarized postsynaptic neurons to presynaptic terminals. Neuron 29:729_ 738.

Okun M, Lampl I (2008) Instantaneous correlation of excitation and inhibition during ongoing and sensory-evoked activities. Nat Neurosci 11:535-537.

Oswald A-MM, Doiron B, Rinzel J, Reyes AD (2009) Spatial profile and differential recruitment of GABAB modulate oscillatory activity in auditory cortex. J Neurosci 29:10321-10334.

Packer AM, Yuste R (2011) Dense, unspecific connectivity of neocortical parvalbumin-positive interneurons: a canonical microcircuit for inhibition? J. Neurosci. 31:13260-13271.

Page CE, Coutellier L (2019) Prefrontal excitatory/inhibitory balance in stress and emotional disorders: Evidence for over-inhibition. Neurosci Biobehav Rev 105:39-51.

Pangratz-Fuehrer S, Hestrin S (2011) Synaptogenesis of electrical and GABAergic synapses of fast-spiking inhibitory neurons in the neocortex. J Neurosci 31:10767-10775.

Patel S, Hillard CJ (2006) Pharmacological evaluation of cannabinoid receptor ligands in a mouse model of anxiety: further evidence for an anxiolytic role for endogenous cannabinoid signaling. J Pharmacol Exp Ther 318:304-311.

Patel S, Kingsley PJ, Mackie K, Marnett LJ, Winder DG (2009) Repeated homotypic stress elevates 2-arachidonoylglycerol levels and enhances short-term endocannabinoid signaling at inhibitory synapses in basolateral amygdala. Neuropsychopharmacology 34:2699-2709.

Patel S, Roelke CT, Rademacher DJ, Cullinan WE, Hillard CJ (2004) Endocannabinoid signaling negatively modulates stress-induced activation of the hypothalamic-pituitaryadrenal axis. Endocrinology 145:5431-5438.

Pearce RA (1993) Physiological evidence for two distinct GABAA responses in rat hippocampus. Neuron 10:189-200.

Petanjek Z, Judaš M, Šimic G, Rasin MR, Uylings HBM, Rakic P, Kostovic I (2011) Extraordinary neoteny of synaptic spines in the human prefrontal cortex. Proc Natl Acad Sci U S A 108:13281-13286.

Pfeffer CK, Xue M, He M, Huang ZJ, Scanziani M (2013) Inhibition of inhibition in visual cortex: the logic of connections between molecularly distinct interneurons. Nat Neurosci 16:10681076.

Pinto DJ, Brumberg JC, Simons DJ (2000) Circuit dynamics and coding strategies in rodent somatosensory cortex. J Neurophysiol 83:1158-1166.

Pitler TA, Alger BE (1992) Postsynaptic spike firing reduces synaptic GABAA responses in hippocampal pyramidal cells. J. Neurosci. 12:4122-4132.

Pitler TA, Alger BE (1994) Depolarization-induced suppression of GABAergic inhibition in rat hippocampal pyramidal cells: $\mathrm{G}$ protein involvement in a presynaptic mechanism. Neuron 13:1447-1455.

Popoli M, Yan Z, McEwen BS, Sanacora G (2011) The stressed synapse: the impact of stress and glucocorticoids on glutamate transmission. Nat Rev Neurosci 13:22-37.

Porter JT, Cauli B, Tsuzuki K, Lambolez B, Rossier J, Audinat E (1999) Selective excitation of subtypes of neocortical interneurons by nicotinic receptors. J Neurosci 19:5228-5235. 
Prescott SA, Koninck $Y$ de (2003) Gain control of firing rate by shunting inhibition: roles of synaptic noise and dendritic saturation. Proc Natl Acad Sci U S A 100:2076-2081.

Prut L, Belzung C (2003) The open field as a paradigm to measure the effects of drugs on anxiety-like behaviors: a review. Eur J Pharmacol 463:3-33.

Rademacher DJ, Meier SE, Shi L, Ho W-SV, Jarrahian A, Hillard CJ (2008) Effects of acute and repeated restraint stress on endocannabinoid content in the amygdala, ventral striatum, and medial prefrontal cortex in mice. Neuropharmacology 54:108-116.

Radley JJ, Rocher AB, Miller M, Janssen WGM, Liston C, Hof PR, McEwen BS, Morrison JH (2006) Repeated stress induces dendritic spine loss in the rat medial prefrontal cortex. Cereb Cortex 16:313-320.

Rakic P (2009) Evolution of the neocortex: a perspective from developmental biology. Nat Rev Neurosci 10:724-735.

Roberto M, Madamba SG, Moore SD, Tallent MK, Siggins GR (2003) Ethanol increases GABAergic transmission at both pre- and postsynaptic sites in rat central amygdala neurons. Proc Natl Acad Sci U S A 100:2053-2058.

Roozendaal B, McEwen BS, Chattarji S (2009) Stress, memory and the amygdala. Nat Rev Neurosci 10:423-433.

Rubino T, Guidali C, Vigano D, Realini N, Valenti M, Massi P, Parolaro D (2008) CB1 receptor stimulation in specific brain areas differently modulate anxiety-related behaviour. Neuropharmacology 54:151-160.

Rubino T, Sala M, Viganò D, Braida D, Castiglioni C, Limonta V, Guidali C, Realini N, Parolaro D (2007) Cellular mechanisms underlying the anxiolytic effect of low doses of peripheral Delta9-tetrahydrocannabinol in rats. Neuropsychopharmacology 32:2036-2045.

Rudy B, Fishell G, Lee S, Hjerling-Leffler J (2011) Three groups of interneurons account for nearly $100 \%$ of neocortical GABAergic neurons. Dev Neurobiol 71:45-61.

Sabatini BL, Regehr WG (1999) Timing of synaptic transmission. Annu Rev Physiol 61:521542.

Scannevin $\mathrm{RH}$, Huganir $\mathrm{RL}$ (2000) Postsynaptic organization and regulation of excitatory synapses. Nat Rev Neurosci 1:133-141.

Schulz JM, Knoflach F, Hernandez M-C, Bischofberger J (2018) Dendrite-targeting interneurons control synaptic NMDA-receptor activation via nonlinear a5-GABAA receptors. Nat Commun 9:3576.

Shepard R, Page CE, Coutellier L (2016) Sensitivity of the prefrontal GABAergic system to chronic stress in male and female mice: Relevance for sex differences in stress-related disorders. Neuroscience 332:1-12.

Shepherd GM, Rowe TB (2017) Neocortical lamination: Insights from neuron types and evolutionary precursors. Front Neuroanat 11:100.

Sigel E, Steinmann ME (2012) Structure, function, and modulation of GABA(A) receptors. J Biol Chem 287:40224-40231.

Simon P, Dupuis R, Costentin J (1994) Thigmotaxis as an index of anxiety in mice. Influence of dopaminergic transmissions. Behav Brain Res 61:59-64. 
Soderstrom K, Leid M, Moore FL, Murray TF (2000) Behaviroal, pharmacological, and molecular characterization of an amphibian cannabinoid receptor. J Neurochem 75:413423.

Sohal VS, Zhang F, Yizhar O, Deisseroth K (2009) Parvalbumin neurons and gamma rhythms enhance cortical circuit performance. Nature 459:698-702.

Spear LP (2000) The adolescent brain and age-related behavioral manifestations. Neuroscience \& Biobehavioral Reviews 24:417-463.

Srinivas S, Watanabe T, Lin CS, William CM, Tanabe Y, Jessell TM, Costantini F (2001) Cre reporter strains produced by targeted insertion of EYFP and ECFP into the ROSA26 locus. BMC Dev Biol 1:4.

Steindel F, Lerner R, Häring M, Ruehle S, Marsicano G, Lutz B, Monory K (2013) Neuron-type specific cannabinoid-mediated $G$ protein signalling in mouse hippocampus. J Neurochem 124:795-807.

Stell BM, Brickley Stephen G., Tang CY, Farrant M, Mody I (2003) Neuroactive steroids reduce neuronal excitability by selectively enhancing tonic inhibition mediated by delta subunitcontaining GABAA receptors. Proc Natl Acad Sci U S A 100:14439-14444.

Stern P, Edwards FA, Sakmann B (1992) Fast and slow components of unitary EPSCs on stellate cells elicited by focal stimulation in slices of rat visual cortex. J Physiol (Lond) 449:247-278.

Sumislawski JJ, Ramikie TS, Patel S (2011) Reversible gating of endocannabinoid plasticity in the amygdala by chronic stress: a potential role for monoacylglycerol lipase inhibition in the prevention of stress-induced behavioral adaptation. Neuropsychopharmacology 36:2750-2761.

Sun Q-Q, Huguenard JR, Prince DA (2006) Barrel cortex microcircuits: thalamocortical feedforward inhibition in spiny stellate cells is mediated by a small number of fast-spiking interneurons. J Neurosci 26:1219-1230.

Szkudlarek HJ, Desai SJ, Renard J, Pereira B, Norris C, Jobson CEL, Rajakumar N, Allman BL, Laviolette SR (2019) $\Delta$-9-Tetrahydrocannabinol and Cannabidiol produce dissociable effects on prefrontal cortical executive function and regulation of affective behaviors. Neuropsychopharmacology 44:817-825.

Tamás G, Buhl EH, Lörincz A, Somogyi P (2000) Proximally targeted GABAergic synapses and gap junctions synchronize cortical interneurons. Nat Neurosci 3:366-371.

Taniguchi H, He M, Wu P, Kim S, Paik R, Sugino K, Kvitsiani D, Kvitsani D, Fu Y, Lu J, Lin Y, Miyoshi G, Shima Y, Fishell G, Nelson SB, Huang ZJ (2011) A resource of Cre driver lines for genetic targeting of GABAergic neurons in cerebral cortex. Neuron 71:995-1013.

Tanimura A, Yamazaki M, Hashimotodani Y, Uchigashima M, Kawata S, Abe M, Kita Y, Hashimoto K, Shimizu T, Watanabe M, Sakimura K, Kano M (2010) The endocannabinoid 2-arachidonoylglycerol produced by diacylglycerol lipase alpha mediates retrograde suppression of synaptic transmission. Neuron 65:320-327.

Terry-McEIrath YM, O'Malley PM, Johnston LD (2009) Reasons for drug use among American youth by consumption level, gender, and race/ethnicity: 1976-2005. J Drug Issues 39:677714. 
Thomson AM, Bannister AP, Hughes DI, Pawelzik H (2000) Differential sensitivity to Zolpidem of IPSPs activated by morphologically identified CA1 interneurons in slices of rat hippocampus. Eur J Neurosci 12:425-436.

Tremblay R, Lee S, Rudy B (2016) GABAergic interneurons in the neocortex: From cellular properties to circuits. Neuron 91:260-292.

Trettel J, Fortin DA, Levine ES (2004) Endocannabinoid signalling selectively targets perisomatic inhibitory inputs to pyramidal neurones in juvenile mouse neocortex. J Physiol (Lond ) 556:95-107.

Turrigiano G (2011) Too many cooks? Intrinsic and synaptic homeostatic mechanisms in cortical circuit refinement. Annu Rev Neurosci 34:89-103.

Uchigashima M, Yamazaki M, Yamasaki M, Tanimura A, Sakimura K, Kano M, Watanabe M (2011) Molecular and morphological configuration for 2-arachidonoylglycerol-mediated retrograde signaling at mossy cell-granule cell synapses in the dentate gyrus. J Neurosci $31: 7700-7714$.

Wall MJ, Usowicz MM (1998) Development of the quantal properties of evoked and spontaneous synaptic currents at a brain synapse. Nat Neurosci 1:675-682.

Wellman CL (2001) Dendritic reorganization in pyramidal neurons in medial prefrontal cortex after chronic corticosterone administration. J Neurobiol 49:245-253.

Whissell PD, Cajanding JD, Fogel N, Kim JC (2015) Comparative density of CCK- and PVGABA cells within the cortex and hippocampus. Front Neuroanat 9:124.

Williams L, Parker H (2001) Alcohol, cannabis, ecstasy and cocaine: drugs of reasoned choice amongst young adult recreational drug users in England. International Journal of Drug Policy 12:397-413.

Wilson RL, Kunos G, Nicoll RA (2001) Presynaptic specificity of endocannabinoid signaling in the hippocampus. Neuron 31:453-462.

Wilson RL, Nicoll RA (2001) Endogenous cannabinoids mediate retrograde signalling at hippocampal synapses. Nature 410:588-592.

Winters BD, Krüger JM, Huang X, Gallaher ZR, Ishikawa M, Czaja K, Krueger JM, Huang YH, Schlüter OM, Dong Y (2012) Cannabinoid receptor 1-expressing neurons in the nucleus accumbens. Proc Natl Acad Sci U S A 109:E2717-25.

Wobbrock JO, Findlater L, Gergle D, Higgins JJ (2011) The aligned rank transform for nonparametric factorial analyses using only anova procedures. In: Proceedings of the 2011 annual conference on Human factors in computing systems - CHI '11 (Tan D, Fitzpatrick G, Gutwin C, Begole B, Kellogg WA, eds), p 143. New York, New York, USA: ACM Press.

Xerri C (2012) Plasticity of cortical maps: multiple triggers for adaptive reorganization following brain damage and spinal cord injury. Neuroscientist 18:133-148.

Xu X, Roby KD, Callaway EM (2010) Immunochemical characterization of inhibitory mouse cortical neurons: three chemically distinct classes of inhibitory cells. J Comp Neurol 518:389-404.

Xue M, Atallah BV, Scanziani M (2014) Equalizing excitation-inhibition ratios across visual cortical neurons. Nature 511:596-600. 
Yang J-M, Zhang J, Yu Y-Q, Duan S, Li X-M (2014) Postnatal development of 2 microcircuits involving fast-spiking interneurons in the mouse prefrontal cortex. Cereb Cortex 24:98109.

Yoshino H, Miyamae T, Hansen G, Zambrowicz B, Flynn M, Pedicord D, Blat Y, Westphal RS, Zaczek R, Lewis DA, Gonzalez-Burgos G (2011) Postsynaptic diacylglycerol lipase mediates retrograde endocannabinoid suppression of inhibition in mouse prefrontal cortex. J Physiol (Lond ) 589:4857-4884.

Yuen EY, Wei J, Liu W, Zhong P, Li X, Yan Z (2012) Repeated stress causes cognitive impairment by suppressing glutamate receptor expression and function in prefrontal cortex. Neuron 73:962-977.

Yuste R, Denk W (1995) Dendritic spines as basic functional units of neuronal integration. Nature 375:682-684.

Zhong P, Wang W, Pan B, Liu X, Zhang Z, Long JZ, Zhang H-t, Cravatt BF, Liu Q-s (2014) Monoacylglycerol lipase inhibition blocks chronic stress-induced depressive-like behaviors via activation of mTOR signaling. Neuropsychopharmacology 39:1763-1776.

Zucker RS, Regehr WG (2002) Short-term synaptic plasticity. Annu Rev Physiol 64:355-405. 


\section{Appendix}

\section{1. $\quad$ Supplementary Figures}

A

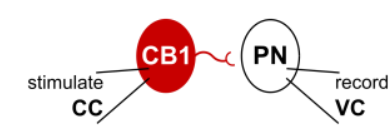

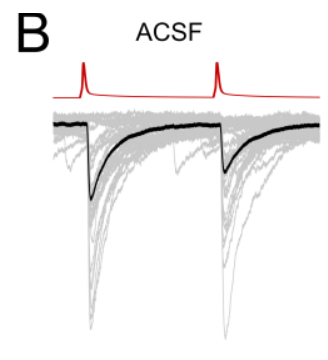

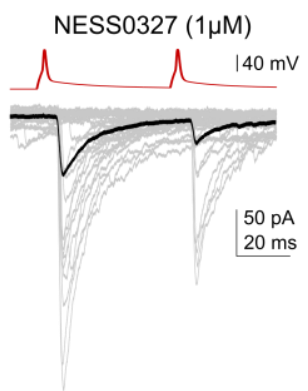

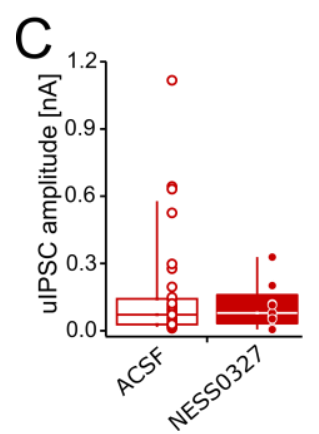
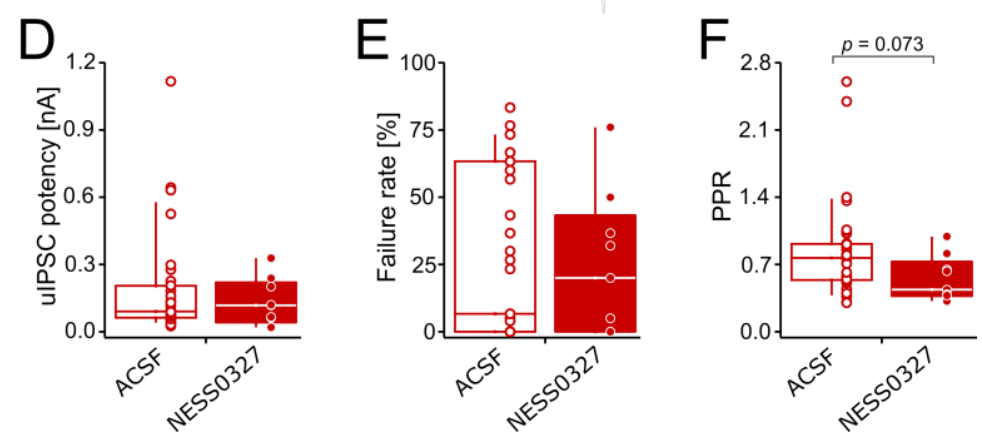

Supplementary Figure S1: The neutral CB1 receptor-selective antagonist NESS0327 does not affect basal synaptic strength and release properties at synapses made by $\mathrm{CB} 1+$ interneurons in prefrontal cortex. A) Illustration of recording configuration. Pairwise recordings were made between a presynaptic CB1+ IN and a postsynaptic PN in mPFC L2/3. B) Example traces of synaptically connected CB1-to-PN pairs in ACSF only and in the presence of the neutral CB1 receptor-selective antagonist NESS0327 (1 $\mu \mathrm{M})$. C-F) Summary graphs depicting (C) the uIPSC amplitude, (D) the uIPSC potency, (E) the failure rate, and (F) the PPR of uIPSCs at connected CB1to-PN pairs in the presence of ACSF only or NESS0327. The presence of NESS0327 did not affect the basal synaptic strength and release properties at CB1-to-PN pairs, as evidenced by the unchanged uIPSC amplitude $(U=-$ $0.03, p=0.976)$, uIPSC potency $(U=-0.33, p=0.743)$, and failure rate $(U=-0.03, p=0.975)$. There was only a trend for a lower PPR in the presence of NESS0327 compared to ACSF only $(U=-1.79, p=0.073)$. ACSF: $\mathrm{n} / \mathrm{m}=34 / 18$; NESS0327: $\mathrm{n} / \mathrm{m}=9 / 5$; Data were analyzed with Mann-Whitney $U$ tests. ${ }^{*} p<0.05$.
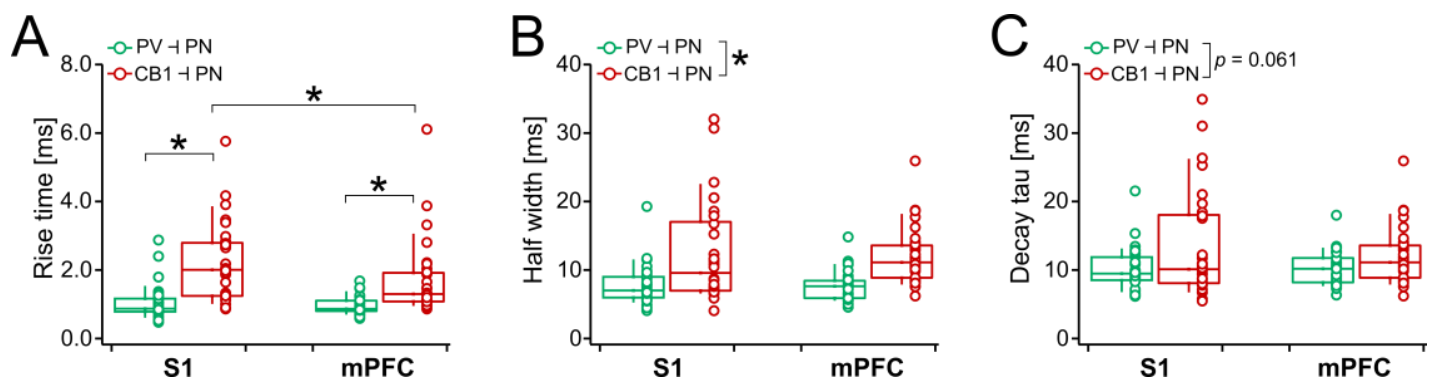

Supplementary Figure S2: PV+ and CB1+ interneurons form GABAergic synapses with different kinetic properties in somatosensory and prefrontal cortex. A-B) Summary graphs depicting $(\mathbf{A})$ the rise time, $(B)$ the half width, and (C) the decay time constant tau of uIPSCs at connected PV-to-PN pairs (green) and CB1-to-PN pairs (red) in S1 and mPFC. uIPSCs generally had longer rise times at CB1-to-PN pairs compared to PV-to-PN pairs in both $S 1(p<0.001)$ and mPFC $(p<0.001)$. However, the differences were smaller in mPFC compared to S1 (cell type: $F_{(1,128)}=77.62, p<0.001$; region: $F_{(1,128)}=8.39, p=0.004$; cell type $x$ region: $\left.F_{(1,128)}=9.49, p=0.003\right)$, due to significantly shorter rise times of uIPSCs in mPFC compared to S1 at CB1-to-PN pairs $(p=0.035)$ but not PV-to-PN pairs $(p=0.927)$. Moreover, uIPSCs of CB1-to-PN pairs generally exhibited longer half widths, irrespective of cortical region (cell type: $F_{(1,128)}=14.81, p<0.001$; region: $F_{(1,128)}=1.70, p=0.195$; cell type x region: $F_{(1,128)}=2.97, p=0.087$ ). Finally, there was a region-independent trend for a longer decay time constant tau of uIPSCs at CB1-to-PN pairs compared to PV-to-PN pairs (cell type: $F_{(1,128)}=3.57, p=0.061$; region: $F(1,128)=0.49, p=0.485$; cell type $\times$ region: $F(1,128)=0.00, p=0.933)$. However, note the broader parameter distributions for the half width and the decay time constant tau with a higher incidence of large values for CB1-to-PN pairs in S1 compared to mPFC. Data are presented as box plots. Whiskers represent the $10^{\text {th }}$ and $90^{\text {th }}$ percentile. Dots represent individual data points. PVto-PN pairs: $n / m=35 / 15$ for $S 1, n / m=33 / 18$ for mPFC; CB1-to-PN pairs: $n / m=30 / 15$ for $S 1, n / m=34 / 18$ for mPFC. Data were analyzed with two-way art-ANOVAs followed by post-hoc Bonferroni Holm-adjusted MannWhitney U tests. * $p<0.05$. 

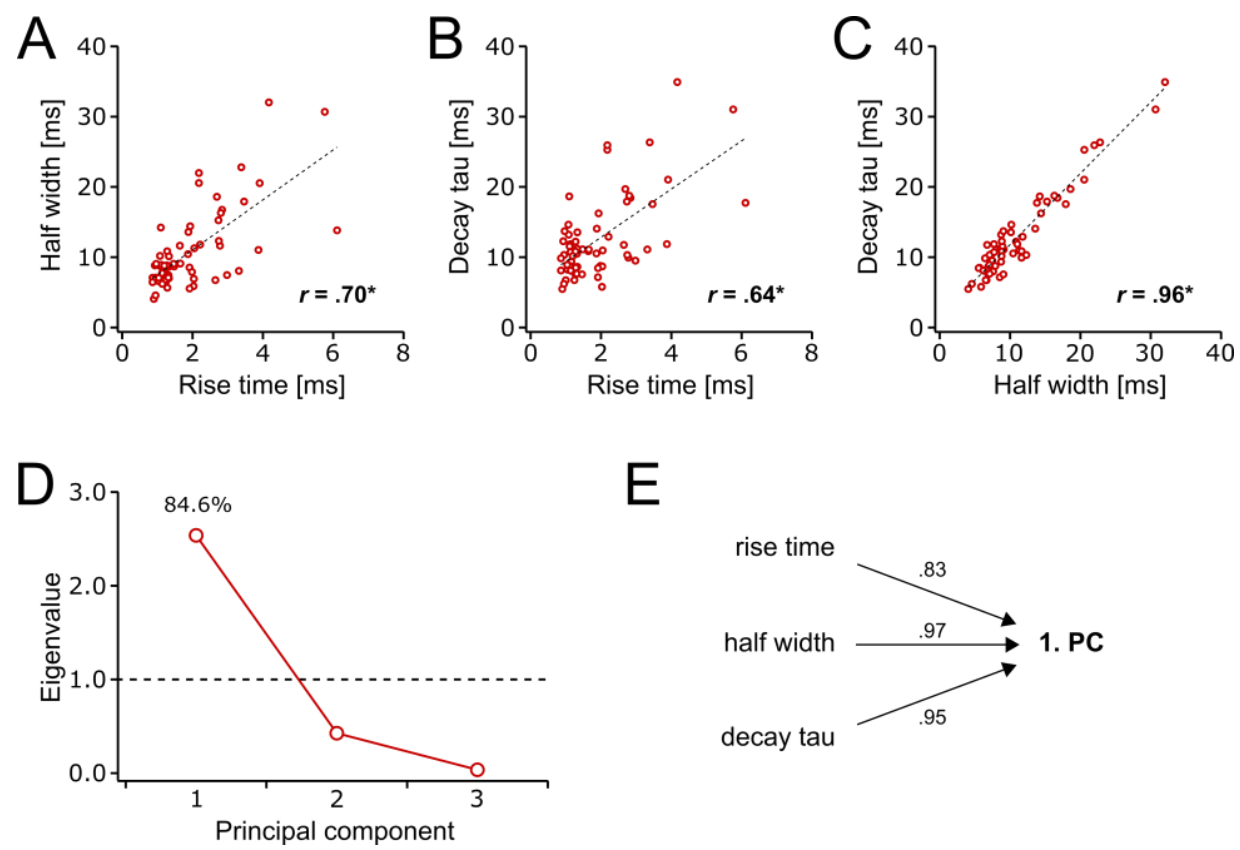

E

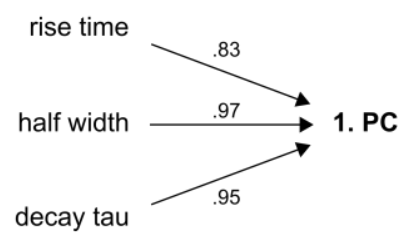

Supplementary Figure S3: Dimensionality reduction of the kinetic properties of GABAergic synapses made by CB1+ interneurons. A-C) Scatterplots depicting the relationships between the different kinetic parameters at CB1-to-PN pairs, collapsed across S1 and mPFC. Significant correlations were obtained between all three kinetic parameters, i.e. (A) the rise time and half width (Pearson's $r=.70, p<0.001$ ), (B) the rise time and the decay time constant tau (Pearson's $r=.64, p<0.001$ ), and (C) the half width and the decay time constant tau (Pearson's $r=$ $.96, p<0.001)$. D) Scree plot depicting the Eigenvalues of principal components obtained from a principal component analysis of the three kinetic parameters. The first principal component with an Eigenvalue $>1.0$ was extracted, which explained $84.6 \%$ of the total variance in the data set. E) Loading diagram depicting the factor loadings of the three kinetic parameters onto the extracted principal component (1. PC). Factor loadings correspond to bivariate Pearson correlations and are indicated at the arrows. $n / m=64 / 33$. ${ }^{*} p<0.05$.
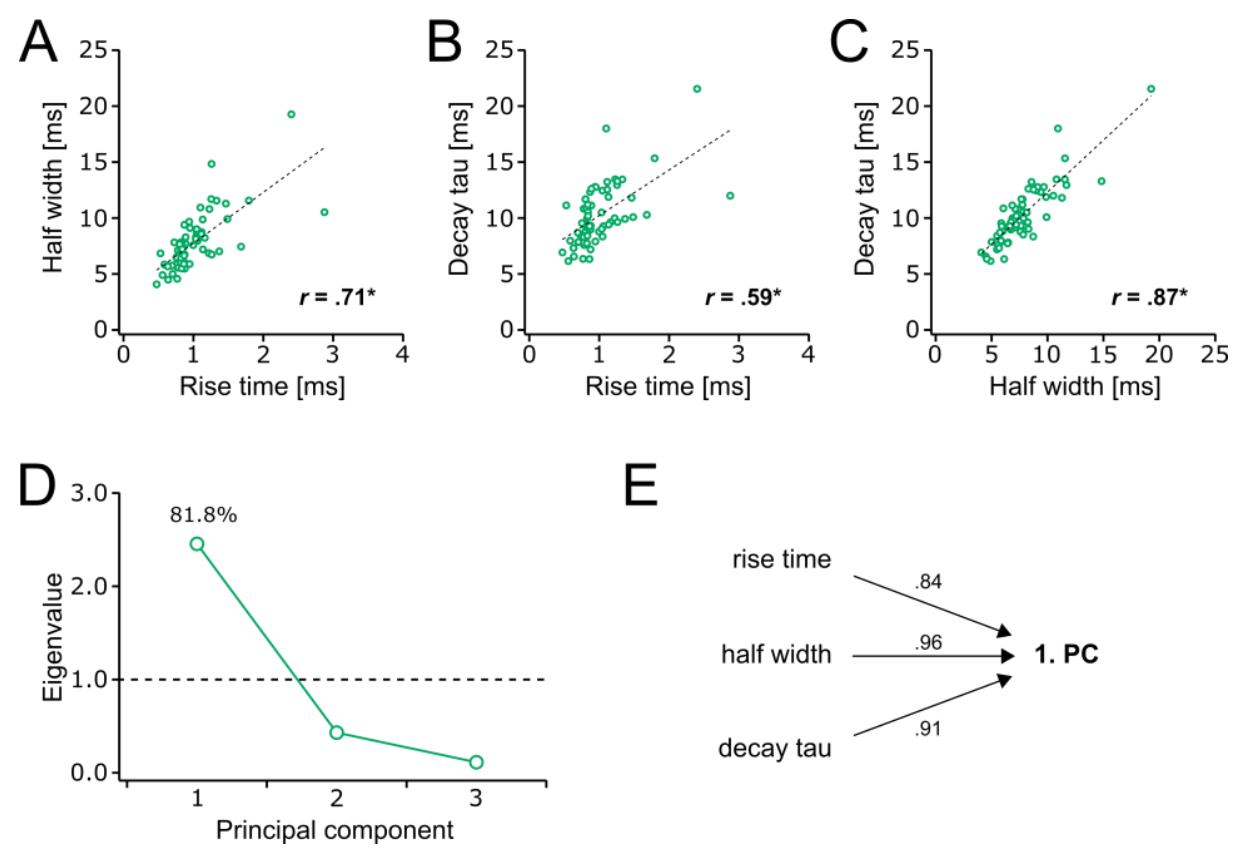

$E$

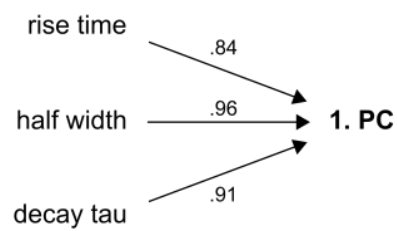

Supplementary Figure S4: Dimensionality reduction of the kinetic properties of GABAergic synapses made by PV+ interneurons. A-C) Scatterplots depicting the relationships between the different kinetic parameters at PVto-PN pairs, collapsed across S1 and mPFC. Significant correlations were obtained between all three kinetic parameters, i.e. (A) the rise time and half width (Pearson's $r=.71, p<0.001$ ), (B) the rise time and the decay time constant tau (Pearson's $r=.59, p<0.001$ ), and (C) the half width and the decay time constant tau (Pearson's $r=$ $.87, p<0.001)$. D) Scree plot depicting the Eigenvalues of principal components obtained from a principal component analysis of the three kinetic parameters. The first principal component with an Eigenvalue $>1.0$ was extracted, which explained $81.8 \%$ of the total variance in the data set. E) Loading diagram depicting the factor loadings of the three kinetic parameters onto the extracted principal component (1. PC). Factor loadings correspond to bivariate Pearson correlations and are indicated at the arrows. $\mathrm{n} / \mathrm{m}=68 / 33$. ${ }^{*} p<0.05$. 

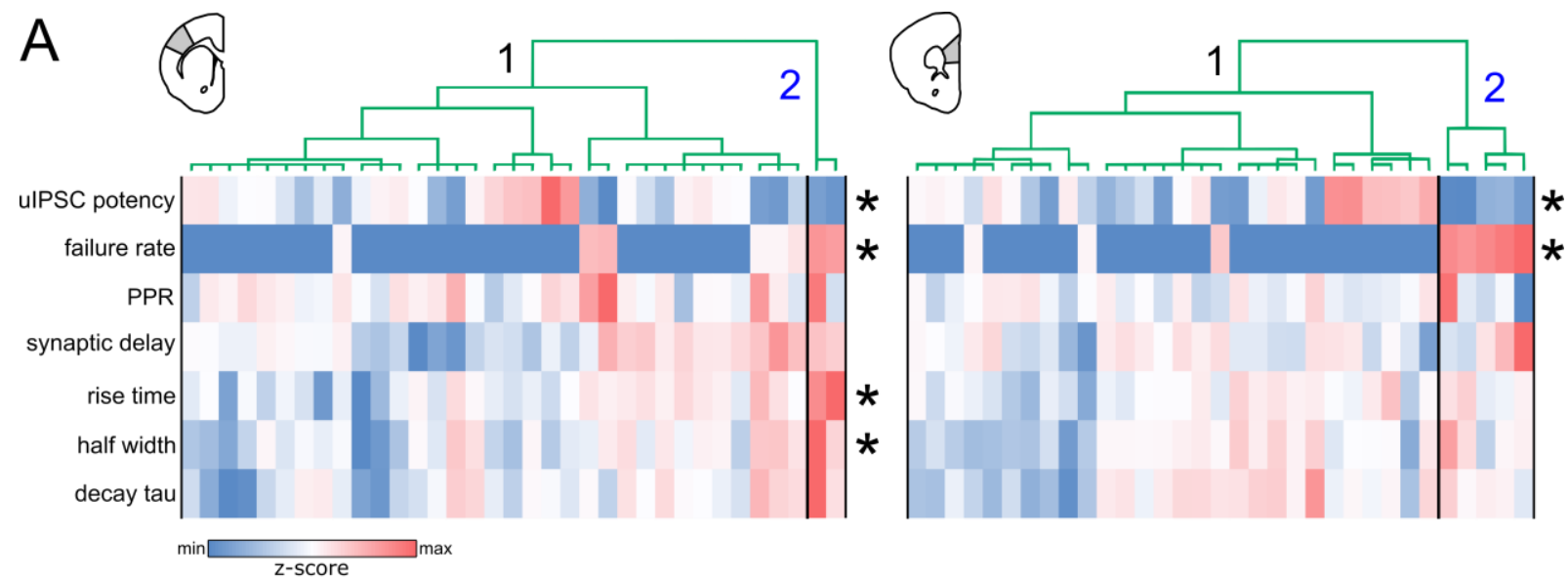

B
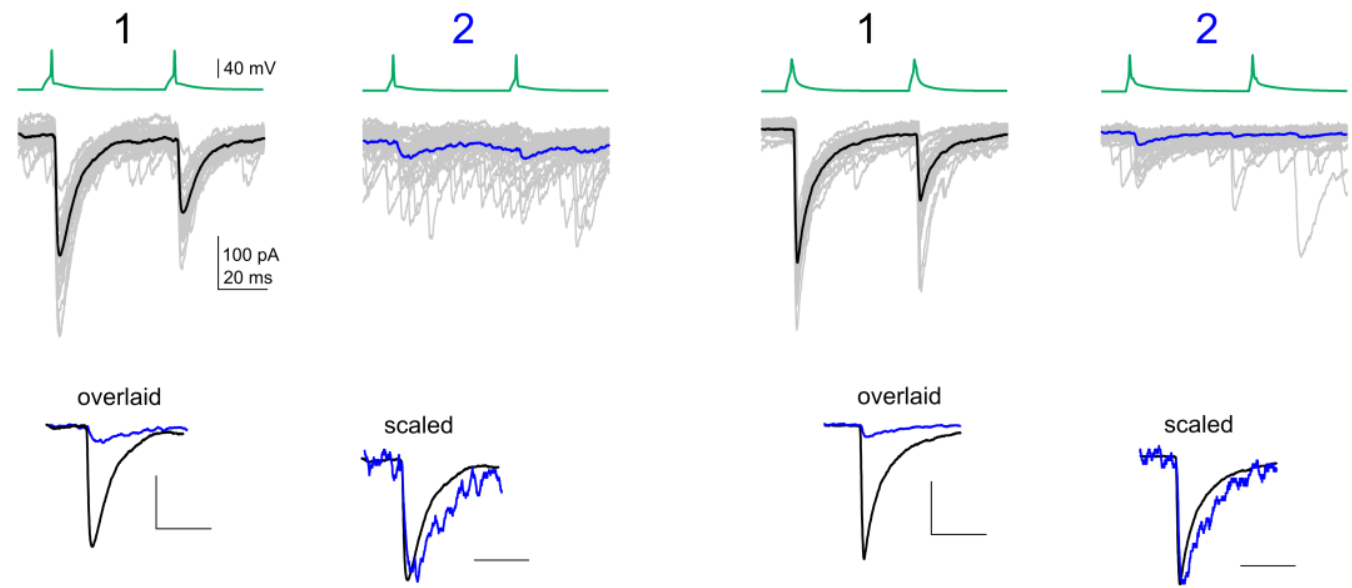

Supplementary Figure S5: PV + interneurons do not form distinct subtypes of GABAergic synapses in either somatosensory or prefrontal cortex. A) Two-cluster solutions obtained from hierarchical cluster analysis for synapses made by PV+ INs in S1 (left) and mPFC (right). Hierarchical cluster analysis was performed using the uIPSC potency, the failure rate, and the synaptic delay, together with the first principal component derived from a principal component analysis of the rise times, half widths, and decay time constants of uIPSCs at PV-to-PN pairs. Upper part: Dendrograms. The two clusters are denoted by 1 and 2. Lower part: Heat maps depicting the synaptic properties of individual PV-to-PN pairs from clusters 1 and 2. Synaptic parameters were z-transformed and then color-coded, with blue representing minimum and red colors representing maximum values. B) Example traces of connected PV-to-PN pairs from cluster 1 and 2 in S1 and mPFC. C) Average uIPSCs recorded in PNs in response to the first presynaptic action potential at PV-to-PN pairs shown in B), overlaid and scaled between cluster 1 (black) and cluster 2 (blue). In both cortical regions, the resulting synapse clusters were generally not very distinctive. PVto-PN pairs were mainly clustered based on their failure rates and generally felt either into a large cluster of reliable synapses (cluster 1 ) or a very small cluster of weak and highly unreliable synapses (cluster 2 ) in both S1 and mPFC. In $S 1$, clusters 1 and 2 only significantly differed in the uIPSC potency $(U=-1.99, p=0.047)$, failure rate $(U=-3.19$, $p=0.001)$, rise time $(U=-2.35, p=0.019)$, and half width of uIPSCs $(U=-2.13, p=0.033$; all other $p$-values $>0.05)$. In mPFC, clusters 1 and 2 only significantly differed in the uIPSC potency $(U=-2.81, p=0.005)$ and failure rate $(U=-$ $4.67, p<0.001$; all other $p$-values $>0.05$ ). Note that although some cluster differences in the kinetic parameters were detected, the shape of scaled uIPSCs was highly comparable between synapses from clusters 1 and 2 in both $S 1$ and mPFC, indicating that their kinetic properties were largely similar (C). S1: $\mathrm{n} / \mathrm{m}=33 / 15$ for cluster $1, \mathrm{n} / \mathrm{m}=2 / 1$ for cluster 2; mPFC: $n / m=28 / 16$ for cluster $1, n / m=5 / 4$ for cluster 2 . Hierarchical cluster analysis was performed using Ward fusion algorithm and squared Euclidian distance as linkage distance. Clusters were compared with Mann-Whitney U tests. ${ }^{*} p<0.05$. 

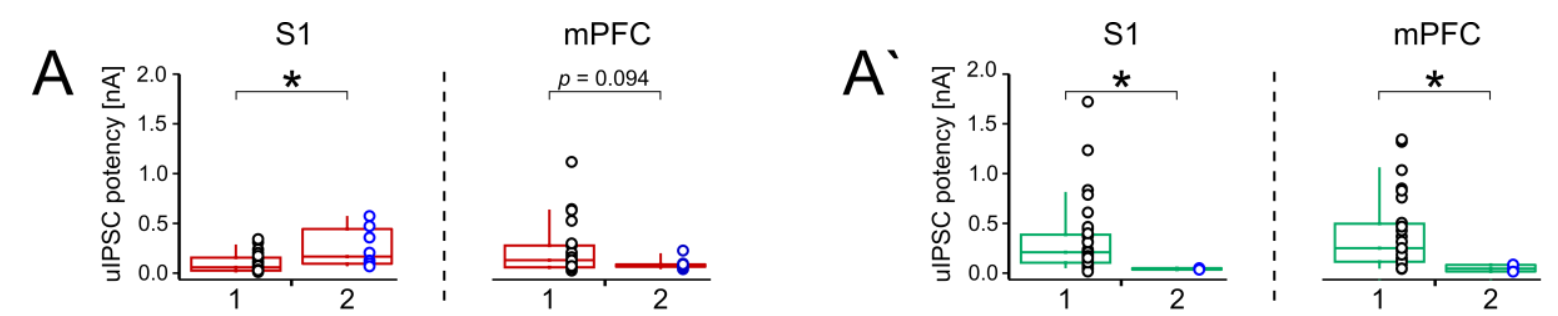

B

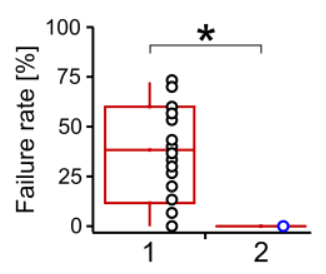

C

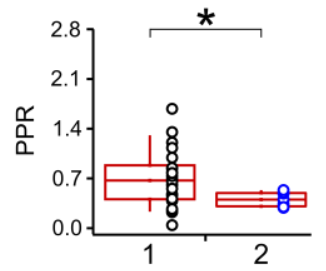

D

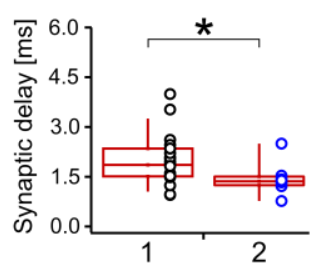

E

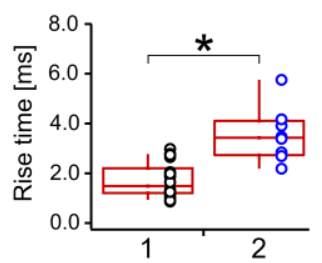

F

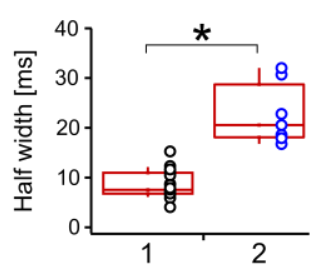

G

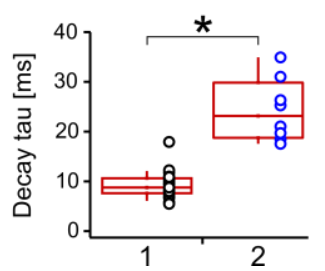

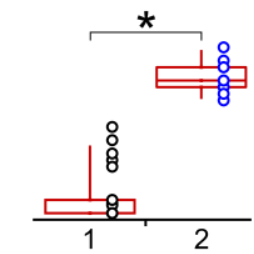
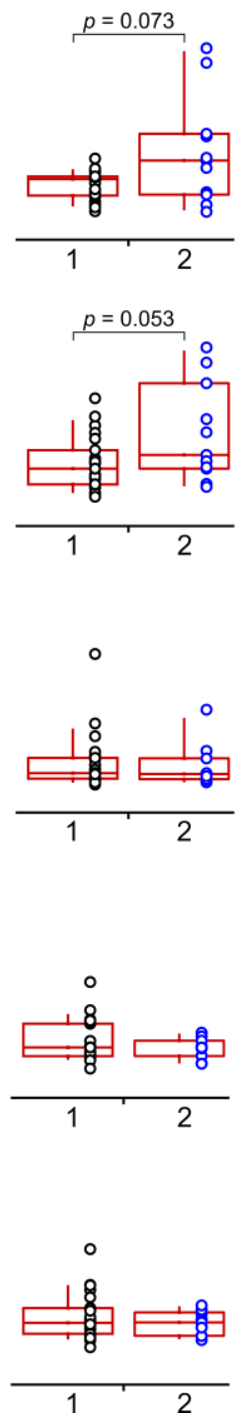

B

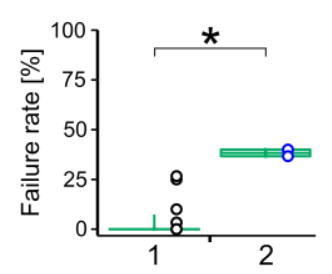

C
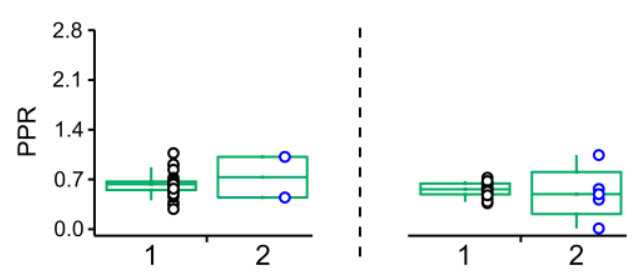

D'
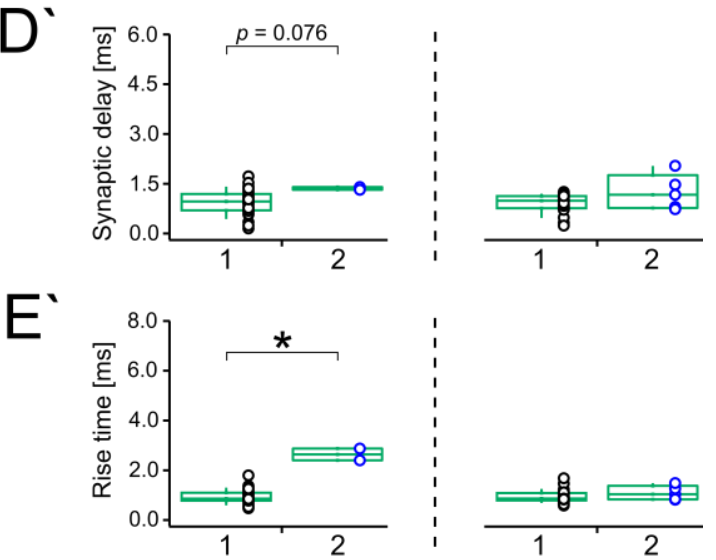

Ev
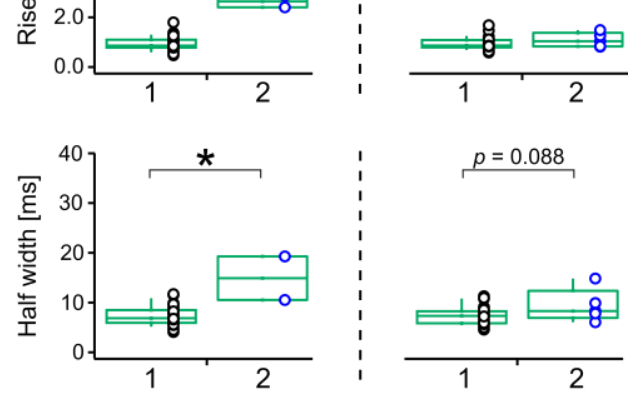

G

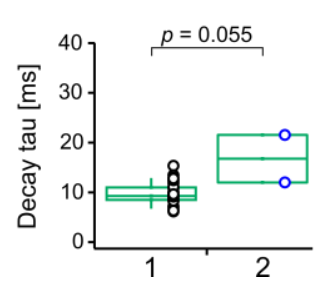

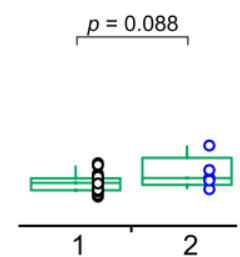

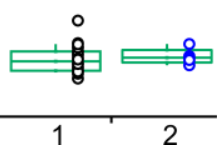

Supplementary Figure S6: Synaptic properties of hierarchical cluster solutions obtained for synapses made by $C B 1+$ and $P V+$ interneurons in somatosensory and prefrontal cortex. A-G') Summary graphs depicting (A$\left.\mathbf{A}^{\prime}\right)$ the uIPSC potency, (B-B') the failure rate, $\left(\mathbf{C}^{\prime} \mathbf{C}^{\prime}\right)$ the PPR, (D-D') the synaptic delay, (E-E') the rise time, (F$\left.\mathbf{F}^{\prime}\right)$ the half width, and (G-G') the decay time constant tau for (A-G) connected CB1-to-PN pairs (red) and ( $\left.\mathbf{A}^{\prime}-\mathbf{G}^{\prime}\right)$ PV-to-PN pairs (green) belonging to synapse clusters 1 and 2 in $S 1$ and mPFC. Note that CB1 -to-PN pairs in mPFC were clustered in a similar manner as PV-to-PN pairs in both cortical regions, with the resulting synapse clusters 1 and 2 mainly differing in their uIPSC potency and failure rate. Data are presented as box plots. Whiskers represent the $10^{\text {th }}$ and $90^{\text {th }}$ percentile. Dots represent individual data points, with synapse cluster 1 highlighted in black and synapse cluster 2 highlighted in blue. CB1-to-PN pairs: $n / m=22 / 14$ for $S 1$ cluster $1, n / m=8 / 5$ for $S 1$ cluster $2, n / m$ $=23 / 16$ for $\mathrm{mPFC}$ cluster $1, \mathrm{n} / \mathrm{m}=11 / 7$ for $\mathrm{mPFC}$ cluster 2 ; PV-to-PN pairs: $\mathrm{n} / \mathrm{m}=33 / 15$ for $\mathrm{S} 1$ cluster $1, \mathrm{n} / \mathrm{m}=2 / 1$ for $\mathrm{S} 1$ cluster $2, \mathrm{n} / \mathrm{m}=28 / 16$ for $\mathrm{mPFC}$ cluster $1, \mathrm{n} / \mathrm{m}=5 / 4$ for $\mathrm{mPFC}$ cluster 2 . Clusters were compared with MannWhitney U tests. ${ }^{*} p<0.05$. 
A
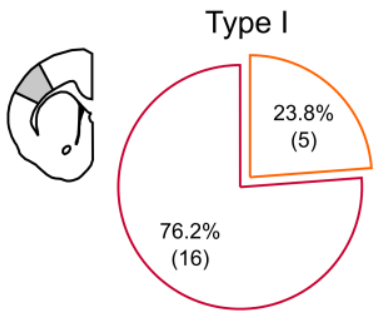

2

Type II

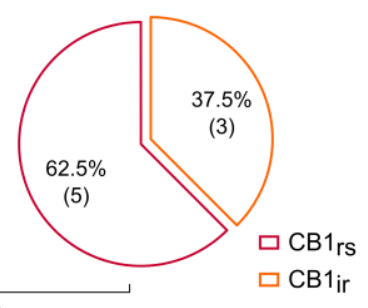

1

Type I: reliable

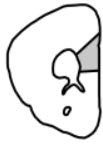

2

Type I: unreliable

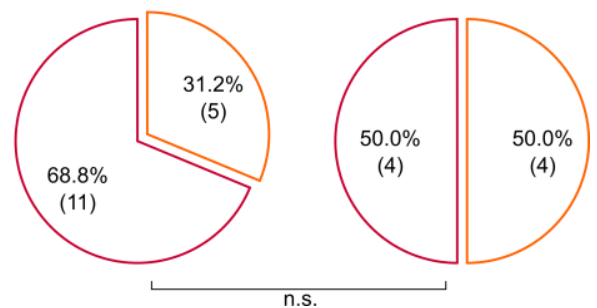

Supplementary Figure S7: Cluster membership does not correlate with the firing properties of CB1+ interneurons in either somatosensory or prefrontal cortex. A-B) Pie charts depicting the proportion of synapses made by regular-spiking $\mathrm{CB} 1+$ INs (CB1 rs; magenta) and irregular-spiking CB1+ INs (CB1 ir; orange) for $\mathbf{A})$ fast typeI (cluster 1) and slow type-II synapses (cluster 2) in S1 and B) fast type-I reliable synapses (cluster 1) and fast typeI unreliable synapses (cluster 2) in mPFC. The percentage values are depicted in the slices. Absolute numbers are given below in the parentheses. In both cortical regions, the proportion of synapses made by CB1 rs and CB1 ir was comparable between clusters 1 and $2\left(X^{2}=0.54, p=0.646\right.$, for $S 1 ; X^{2}=0.80, p=0.412$, for mPFC). Data were analyzed with Fisher's exact test. ${ }^{*} p<0.05$. 


\subsection{List of chemicals and reagents}

Adenosine 5'-triphosphate magnesium salt Sigma-Aldrich, St. Louis, USA (Mg-ATP)

Agarose

Carl Roth, Karlsruhe, Germany

Calcium chloride dihydrate $\left(\mathrm{CaCl}_{2}{ }^{*} 2 \mathrm{H}_{2} \mathrm{O}\right)$

Carl Roth, Karlsruhe, Germany

Cesium chloride $(\mathrm{CsCl})$

Carl Roth, Karlsruhe, Germany

Cesium gluconate (Cs-gluconate)

Hello Bio, Bristol, UK

Cesium hydroxide solution $(\mathrm{CsOH})$

(50 wt. \% in $\mathrm{H}_{2} \mathrm{O}$ )

Sigma-Aldrich, St. Louis, USA

Cesium methanesulfonate (CsMeS)

Sigma-Aldrich, St. Louis, USA

Choline bicarbonate $\left(\sim 80 \%\right.$ in $\left.\mathrm{H}_{2} \mathrm{O}\right)$

Sigma-Aldrich, St. Louis, USA

DABCO

Sigma-Aldrich, St. Louis, USA

Dimethyl sulfoxide (DMSO)

Carl Roth, Karlsruhe, Germany

D-(+)-Glucose

Merck, Kenilworth, USA

Ethanol (100\%)

Carl Roth, Karlsruhe, Germany

EGTA

Carl Roth, Karlsruhe, Germany

FastGene® Optima HotStart ReadyMix

Forene 100\% (Isoflurane)

NIPPON Genetics Europe, Düren, Germany

Gelatine

AbbVie, North Chicago, USA

Carl Roth, Karlsruhe, Germany

GeneRuler 100 bp Plus DNA Ladder

Thermo Fischer Scientific, Waltham, USA

Glycerol

Carl Roth, Karlsruhe, Germany

Guanosine 5'-triphosphate sodium salt hydrate (Na-GTP)

Sigma-Aldrich, St. Louis, USA

HEPES

Carl Roth, Karlsruhe, Germany

Hydrochloric acid fuming $37 \%$

Carl Roth, Karlsruhe, Germany

Magnesium chloride hexahydrate

$\left(\mathrm{MgCl}_{2}{ }^{*} 6 \mathrm{H}_{2} \mathrm{O}\right)$

Carl Roth, Karlsruhe, Germany

Magnesium sulfate heptahydrate $\left(\mathrm{MgSO}_{4}{ }^{*} 7 \mathrm{H}_{2} \mathrm{O}\right)$

Carl Roth, Karlsruhe, Germany 
Midori Green Xtra

MOWIOL

N-methyl-D-glucamine (NMDG)

Nonident P40 (NP40)

Paraformaldehyde (PFA)

Phosphocreatine disodium salt

(phosphocreatine- $\mathrm{Na}_{2}$ )

Potassium chloride ( $\mathrm{KCl})$

Potassium dihydrogen phosphate $\left(\mathrm{KH}_{2} \mathrm{PO}_{4}\right)$

Potassium gluconate (K-gluconate)

Potassium hydroxide $(\mathrm{KOH})$

Primers

Proteinase $\mathrm{K}(20 \mathrm{mg} / \mathrm{ml})$

Sodium chloride $(\mathrm{NaCl})$

Sodium phosphate monobasic monohydrate $\left(\mathrm{NaH}_{2} \mathrm{PO}_{4}\right)$

Sodium bicarbonate $\left(\mathrm{NaHCO}_{3}\right)$

Sodium phosphate dibasic $\left(\mathrm{Na}_{2} \mathrm{HPO}_{4}\right)$

Sodium tetraborate decahydrate

$\left(\mathrm{Na}_{2} \mathrm{~B}_{4} \mathrm{O}_{7}\right.$ * $\left.10 \mathrm{H}_{2} \mathrm{O}\right)$

Tetraethylammonium chloride (TEA-Cl)

Tris

Tween 20

QX 314 bromide (QX314-Br)

Lidocaine N-ethyl chloride (QX314-Cl)
NIPPON Genetics Europe, Düren, Germany

AppliChem, Darmstadt, Germany

Sigma-Aldrich, St. Louis, USA

Fluka, Buchs, Switzerland

Carl Roth, Karlsruhe, Germany

Sigma-Aldrich, St. Louis, USA

Sigma-Aldrich, St. Louis, USA;

Carl Roth, Karlsruhe, Germany

Carl Roth, Karlsruhe, Germany

Sigma-Aldrich, St. Louis, USA

Carl Roth, Karlsruhe, Germany

MPI-EM, Göttingen, Germany

Ambion, Foster City, USA

Carl Roth, Karlsruhe, Germany

Sigma-Aldrich, St. Louis, USA

Sigma-Aldrich, St. Louis, USA

Sigma-Aldrich, St. Louis, USA

Sigma-Aldrich, St. Louis, USA

Fluka, Buchs, Switzerland

Carl Roth, Karlsruhe, Germany

Carl Roth, Karlsruhe, Germany

Hello Bio, Bristol, UK

Sigma-Aldrich, St. Louis, USA 


\subsection{List of drugs}

Corticosterone

D-AP5

NBQX disodium salt

NESS0327

Picrotoxin

Tetrodotoxin citrate
Hello Bio, Bristol, UK

Hello Bio, Bristol, UK

Hello Bio, Bristol, UK

Caymen Chemical, Ann Arbor, USA

Abcam/Ascent Scientific, Cambridge, UK

Hello Bio, Bristol, UK 


\section{Acknowledgements}

I first want to express my gratitude towards my supervisor Dr. Dr. Oliver Schlüter for giving me the opportunity to work on this interesting $\mathrm{PhD}$ project and for his permanent support throughout the last four years.

I further would like to thank my thesis committee members Prof. Dr. André Fischer and Prof. Dr. Hannelore Ehrenreich for their support and valuable input during the TAC meetings.

Many thanks go of course to all the past and present members of the Molecular Neurobiology lab, especially our technician Sandra Gebauer for her intense commitment and for taking care of us and the lab. I additionally want to thank Myrto Panopoulou for the fruitful and amusing discussions, for joining me throughout my $\mathrm{PhD}$, and for providing help whenever I needed it. Finally, I want to thank all the former members of lab, Plinio Favaro, Lei Cui, Joana Duda, Man Ho Wong and Yanling Liu.

I also want to thank my family, especially my parents, for always supporting and helping me whenever I had problems. I don't know what I would have done without you.

The biggest thanks by far yet goes to my girlfriend Nathalie Heiderich, without whose permanent support and help I wouldn't stand here. Thank you for always being there for me. Thank you for all the times you took care of all the things and duties, when I was too lazy. Thank you for having endured all the times I was in a bad mood, treated you unfairly, or grunted at you for no obvious reasons. Thank you for always standing by my side. Thank you for giving me a place, where I always feel home. Thank you for helping me grow up and become a better person. I love you. 